\title{
A Presentation of Lie Tori of Type $B_{\ell}$
}

\author{
By
}

Malihe YousofZADEH*

\begin{abstract}
We give a finite presentation of the universal covering algebra of a Lie torus of type $B_{\ell}, \ell \geq 3$.
\end{abstract}

\section{$\S 0 . \quad$ Introduction}

For a complex finite dimensional simple Lie algebra $\mathcal{G}$ and a field $\mathbb{K}$, one can define a Lie algebra $\mathcal{G}(\mathbb{K}):=\mathcal{G}_{\mathbb{Z}} \otimes_{\mathbb{Z}} \mathbb{K}$ over $\mathbb{K}$ where $\mathcal{G}_{\mathbb{Z}}$ is the Chevalley $\mathbb{Z}$ form of $\mathcal{G}$ with respect to a given Chevalley basis of $\mathcal{G}[\mathrm{Ch}]$. In the case that the rank of $\mathcal{G}$ is greater than 1 and $\operatorname{ch}(\mathbb{K}) \neq 2,3$, Stienberg [St] proves that $\mathcal{G}(\mathbb{K})$ is centrally closed and gives a presentation of $\mathcal{G}(\mathbb{K})$ by generators and relations. Kassel $[\mathrm{K}]$ generalizes this concept by considering a unital commutative algebra $A$ over a commutative ring $R$ in place of the field $\mathbb{K}$ and defines the Lie algebra $\mathcal{G}(A):=\mathcal{G}_{\mathbb{Z}} \otimes_{\mathbb{Z}} A$ over $R$. He proves that the universal covering algebra of $\mathcal{G}(A)$ is $\tilde{\mathcal{G}}(A):=\mathcal{G}(A) \oplus C$ where $C$ is linearly isomorphic to $\Omega_{A}^{1} / d A$, the module of Kähler differentials of $A$ modulo exact forms. He also gives a presentation of $\tilde{\mathcal{G}}(A)$ by generators and relations. When $R=\mathbb{C}$ and $A$ is the algebra of Laurent polynomials in $n$-variables, the algebra $\tilde{\mathcal{G}}(A)$ is called, by Moody, Rao and Yokonuma [MRY], an $n$-toroidal Lie algebra. They give an abstract infinite presentation of a 2 -toroidal Lie algebra in terms of generators and relations involving the extended Cartan matrix of $\mathcal{G}$. They use their presentation to construct a great number of representations of $\tilde{\mathcal{G}}\left(\mathbb{C}\left[t_{1}^{ \pm 1}, t_{2}^{ \pm 1}\right]\right)$ for a simply laced algebra $\mathcal{G}$. Saito and Yoshii $[\mathrm{SaY}]$ introduce a class of Lie algebras whose

\footnotetext{
Communicated by K. Saito. Received September 26, 2006. Revised April 13, 2007.

2000 Mathematics Subject Classification(s): 17B65; Secondary 17B67.

*University of Isfahan, Isfahan, P.O.Box 81745-163, Iran.

e-mail: ma.yousofzadeh@math.ui.ac.ir.
}

(c) 2008 Research Institute for Mathematical Sciences, Kyoto University. All rights reserved. 
cores are 2-toroidal Lie algebras. They call their class elliptic Lie algebras as they are used in the study of elliptic singularities. They give a Serre-type presentation of a simply laced elliptic Lie algebra in term of the elliptic Dynkin diagram $(R, G)$ attached to its elliptic root system $R$ (an extended affine root system of nullity 2) with marking $G$ which is a rank 1 subspace of the radical of the semi-positive symmetric bilinear form defining $R$. Yamane [Ya] extends the presentation given by Saito and Yoshii to elliptic Lie algebras in general. More precisely, he gives a Serre-type theorem for the elliptic Lie algebras associated to the (reduced marked) elliptic root systems with rank greater than 2. A toroidal Lie algebra is centrally isogenous to the centerless core of an extended affine Lie algebra [AABGP, Chapter III] which is in turn a Lie torus [Yo2]. Now the question is whether one could find a (finite) presentation of the universal covering algebra of a Lie torus for a given nullity and type. In this work we give an affirmative answer to this question for Lie tori of type $B_{\ell}(\ell \geq 3)$. The nature of our presentation highly depends on generalized Tits construction from which the Lie algebras graded by root systems of type $B_{\ell}(\ell \geq 3), F_{4}$ and $G_{2}$ arise [BZ, Section 3].

\section{§1. Preparation}

Throughout this work all vector spaces are considered over the field of complex numbers $\mathbb{C}$ and all tensor products are taken over $\mathbb{C}$. We denote the dual space of a vector space $V$ by $V^{\star}$. If a finite dimensional vector space $V$ is equipped with a non-degenerate symmetric bilinear form, then for $\alpha \in V^{\star}$, we take $t_{\alpha}$ to be the unique element in $V$ representing $\alpha$ through the form. Also for an algebra $\mathcal{A}, Z(\mathcal{A})$ denotes the center of $\mathcal{A}$. All modules over a unital algebra are considered to be unital. For elements $x_{1}, \ldots, x_{n}$ in a Lie algebra $(\mathcal{G},[\cdot, \cdot])$, we set $\left[x_{n}, \ldots, x_{1}\right]$ to be $\left[x_{n}, \ldots,\left[x_{3},\left[x_{2}, x_{1}\right]\right] \ldots\right]$. Also for a finite dimensional Lie algebra $\mathcal{G}$, define $\kappa(\cdot, \cdot)$ to be the Killing form on $\mathcal{G}$. We denote by $M_{m, n}$, the set of all linear transformations from an $n$-dimensional vector space to an $m$-dimensional vector space or equivalently the set of all $m \times n$ matrices over $\mathbb{C}$. For $X \in M_{m, n}$, let $X^{t}$ be the transposition of $X$. For $\ell \in \mathbb{N}$ and $1 \leq r, s \leq \ell$, by $e_{r, s}$, we mean an element of $M_{\ell, \ell}$ having 1 in $(r, s)$ position and 0 elsewhere. We refer to a finite root system as a subset $\Delta$ of a vector space so that $0 \in \Delta$ and $\Delta \backslash\{0\}$ is a finite root system in the sense of [Bo]. For a finite root system $\Delta$, we set $\Delta^{\times}:=\Delta \backslash\{0\}$.

Let $\ell \in \mathbb{Z}^{\geq 2}$ and $\mathcal{V}$ be a $(2 \ell+1)$-dimensional vector space over the field $\mathbb{C}$, also let $I$ be the identity matrix of rank $\ell$. Take $u$ to be the non-degenerate 
symmetric bilinear form on $\mathcal{V}$ whose matrix is $s=\left(\begin{array}{lll}0 & I & 0 \\ I & 0 & 0 \\ 0 & 0 & 1\end{array}\right)$. Then there exists a basis $\left\{v_{1}, \ldots, v_{2 \ell+1}\right\}$ for $\mathcal{V}$ such that

$$
u\left(v_{i}, v_{\ell+i}\right)=1 \text { for } 1 \leq i \leq \ell, u\left(v_{2 \ell+1}, v_{2 \ell+1}\right)=1 \text { and } u\left(v_{i}, v_{j}\right)=0 \text { otherwise. }
$$

The algebra $\mathcal{G}$, consisting of all endomorphisms $X$ of $\mathcal{V}$ which are skewsymmetric with respect to $u$ i.e. $u(X(v), w)=-u(v, X(w))$ for $v, w \in \mathcal{V}$, is a finite dimensional simple Lie algebra of type $B_{\ell}$ [J, Theorem IV.6.7]. Set $H_{i}:=e_{i, i}-e_{\ell+i, \ell+i}$ for $1 \leq i \leq \ell$. Then $H=\oplus_{i=1}^{\ell} \mathbb{C} H_{i}$ is a Cartan subalgebra of $\mathcal{G}[\mathrm{J}, \S \mathrm{IV} .6]$. For $1 \leq i \leq \ell$, define $\varepsilon_{i} \in H^{\star}$ such that $\varepsilon_{i}\left(H_{j}\right)=\delta_{i, j}$ for $1 \leq j \leq \ell$. Up to isomorphism $\mathcal{G}$ is the unique finite dimensional irreducible $\mathcal{G}$-module of highest weight $\varepsilon_{1}+\varepsilon_{2}$ (see $[\mathrm{H}]$ for the definition of a highest weight) and $\mathcal{V}$ is the unique finite dimensional irreducible $\mathcal{G}$-module of highest weight $\varepsilon_{1}$. We refer to $\mathcal{V}$ as the short highest weight module of $\mathcal{G}$.

One can see that $\mathcal{V}$ and $\mathcal{G}$ are $H$-modules admitting weight space decompositions as follows:

$$
\begin{gathered}
\mathcal{V}=\mathcal{V}_{0} \oplus \sum_{i=1}^{\ell} \mathcal{V}_{ \pm \varepsilon_{i}} \text { and } \\
\mathcal{G}=H \oplus \sum_{i=1}^{\ell} \mathcal{G}_{ \pm \varepsilon_{i}} \oplus \sum_{1 \leq i \neq j \leq \ell} \mathcal{G}_{ \pm\left(\varepsilon_{i} \pm \varepsilon_{j}\right)} \text { with } \mathcal{G}_{0}=H
\end{gathered}
$$

where

$$
\mathcal{V}_{0}=\mathbb{C} v_{2 \ell+1}, \mathcal{V}_{\varepsilon_{i}}=\mathbb{C} v_{i}, \mathcal{V}_{-\varepsilon_{i}}=\mathbb{C} v_{\ell+i} ; 1 \leq i \leq \ell
$$

and

$$
\begin{aligned}
\mathcal{G}_{\varepsilon_{i}}=\mathbb{C}\left(e_{2 \ell+1, \ell+i}-e_{i, 2 \ell+1}\right), \mathcal{G}_{-\varepsilon_{i}}=\mathbb{C}\left(e_{2 \ell+1, i}-e_{\ell+i, 2 \ell+1}\right), \\
\mathcal{G}_{\varepsilon_{i}+\varepsilon_{j}}=\mathbb{C}\left(e_{i, \ell+j}-e_{j, \ell+i}\right), \mathcal{G}_{-\varepsilon_{i}-\varepsilon_{j}}=\mathbb{C}\left(e_{\ell+i, j}-e_{\ell+j, i}\right), \\
\mathcal{G}_{\varepsilon_{i}-\varepsilon_{j}}=\mathbb{C}\left(e_{i, j}-e_{\ell+j, \ell+i}\right) ; 1 \leq i \neq j \leq \ell .
\end{aligned}
$$

So $\Phi:=\{0\} \cup\left\{ \pm \varepsilon_{i}, \pm\left(\varepsilon_{i} \pm \varepsilon_{j}\right) \mid 1 \leq i \neq j \leq \ell\right\}$ is the root system of $\mathcal{G}$ and $\{0\} \cup \Phi_{s h}=\{0\} \cup\left\{ \pm \varepsilon_{i}\right\}_{i=1}^{\ell}$ is the set of weights of $\mathcal{V}$. Now set $\alpha_{\ell}:=\varepsilon_{\ell}$ and $\alpha_{i}:=\varepsilon_{i}-\varepsilon_{i+1}$ for $1 \leq i \leq \ell-1$. One can easily check that $\left\{\alpha_{i}\right\}_{i=1}^{\ell}$ is a base of $\Phi$. Now let $1 \leq i, j \leq \ell$, then $\kappa\left(H_{i}, H_{j}\right)=4 \delta_{i, j}$, so $t_{\varepsilon_{i}}=H_{i} / 4$. For $\alpha \in \Phi^{\times}$, set $h_{\alpha}:=2 t_{\alpha} / \kappa\left(t_{\alpha}, t_{\alpha}\right)$. Then we have

$$
h_{\varepsilon_{i}}=2 H_{i}, \quad 1 \leq i \leq \ell \quad \text { and } \quad h_{\varepsilon_{i} \pm \varepsilon_{j}}=H_{i} \pm H_{j}, \quad 1 \leq i \neq j \leq \ell .
$$


Now set

$$
\begin{aligned}
& e_{\varepsilon_{i}}:=e_{2 \ell+1, \ell+i}-e_{i, 2 \ell+1}, f_{\varepsilon_{i}}:=2\left(e_{\ell+i, 2 \ell+1}-e_{2 \ell+1, i}\right), \quad 1 \leq i \leq \ell, \\
& e_{\varepsilon_{i}+\varepsilon_{j}}:=e_{i, \ell+j}-e_{j, \ell+i}, f_{\varepsilon_{i}+\varepsilon_{j}}:=e_{\ell+j, i}-e_{\ell+i, j}, \quad 1 \leq i<j \leq \ell, \\
& e_{\varepsilon_{i}-\varepsilon_{j}}:=e_{i, j}-e_{\ell+j, \ell+i}, f_{\varepsilon_{i}-\varepsilon_{j}}:=e_{j, i}-e_{\ell+i, \ell+j}, \quad 1 \leq i \leq 1, \\
& h_{i}:=h_{\varepsilon_{i}-\varepsilon_{i+1}}, e_{i}:=e_{\varepsilon_{i}-\varepsilon_{i+1}}, f_{i}:=f_{\varepsilon_{i}-\varepsilon_{i+1}}, \quad 1 \leq i \leq \ell-f_{\ell}:=f_{\varepsilon_{\ell}} .
\end{aligned}
$$

By an easy computation we have the following lemma:

Lemma 1.1. For $\alpha \in\left\{\varepsilon_{i}\right\}_{i=1}^{\ell} \cup\left\{\varepsilon_{i} \pm \varepsilon_{j}\right\}_{1 \leq i<j \leq \ell},\left(e_{\alpha}, h_{\alpha}, f_{\alpha}\right)$ is an $\mathfrak{s l}_{2}$-triple.

Now let $1 \leq i \leq \ell$. It is easy to check that

$$
\begin{aligned}
& \text { (i) } f_{i} \cdot v_{j}= \begin{cases}\delta_{i, j} v_{i+1} & i \neq \ell, 1 \leq j \leq \ell, \\
-2 \delta_{\ell, j} v_{2 \ell+1} & i=\ell, 1 \leq j \leq \ell, \\
2 \delta_{i, \ell} v_{2 \ell} & j=2 \ell+1, \\
-\delta_{i+1, j-\ell} v_{\ell+i} \ell+1 \leq j \leq 2 \ell,\end{cases} \\
& \text { (ii) } f_{j} \cdot f_{i} \cdots f_{2} \cdot f_{1} \cdot v_{1}=0, \quad i \neq \ell, 1 \leq j \neq i+1 \leq \ell, \\
& f_{j} \cdot f_{i} \cdots f_{\ell-1} \cdot f_{\ell} \cdot v_{2 \ell+1}=0, \quad 1 \leq j \neq i-1 \leq \ell .
\end{aligned}
$$

This together with (1.3) implies that

$$
\begin{aligned}
\mathcal{V}_{\varepsilon_{i}} & =\mathbb{C} f_{i-1} \cdot f_{i-2} \cdots f_{2} \cdot f_{1} \cdot v_{1}, \quad 2 \leq i \leq \ell, \\
\mathcal{V}_{0} & =\mathbb{C} f_{\ell} \cdot f_{\ell-1} \cdots f_{2} \cdot f_{1} \cdot v_{1}, \\
\mathcal{V}_{-\varepsilon_{i}} & =\mathbb{C} f_{i} \cdot f_{i+1} \cdots f_{\ell-1} \cdot f_{\ell} \cdot v_{2 \ell+1}, \quad 1 \leq i \leq \ell .
\end{aligned}
$$

Lemma 1.2. If $1 \leq i \leq \ell$, then $f_{i} \cdots f_{2} \cdot f_{1} \cdot v_{1}=\left[f_{i}, \ldots, f_{2}, f_{1}\right] \cdot v_{1}$.

Proof. Using induction on $i$, we are done.

Lemma 1.3. Set $f:=\left[f_{\ell}, \ldots, f_{1}\right]$ and let $2 \leq i \leq \ell$. Then we have $f_{i} \cdot f_{i+1} \cdots f_{\ell} \cdot f \cdot v_{1}=\left[f_{i}, f_{i+1}, \ldots, f_{\ell}, f\right] \cdot v_{1}$.

Proof. Using induction on $\ell-i$, we are done.

Definition 1.1. $\quad$ Let $B$ be a unital commutative associative algebra, $\mathcal{W}$ be a $B$-module and $g: \mathcal{W} \times \mathcal{W} \longrightarrow B$ be a symmetric $B$-bilinear form on $\mathcal{W}$. Then $\operatorname{Cliff}(g):=B 1 \oplus \mathcal{W}$ with the multiplication

$$
(b 1+w) \cdot\left(b^{\prime} 1+w^{\prime}\right)=b b^{\prime} 1+g\left(w, w^{\prime}\right) 1+b w^{\prime}+b^{\prime} w ; w, w^{\prime} \in W, b, b^{\prime} \in B
$$

is a Jordan algebra called the Clifford Jordan algebra of $g$. 
Now consider the Clifford Jordan algebra $\operatorname{Cliff}(g)=B 1 \oplus \mathcal{W}$ for a unital commutative associative algebra $B$, a $B$-module $\mathcal{W}$ and a symmetric $B-$ bilinear form $g: \mathcal{W} \times \mathcal{W} \longrightarrow B$. For $a, a^{\prime} \in \operatorname{Cliff}(g)$, define $d_{a, a^{\prime}}:=\mathrm{L}_{a} \mathrm{~L}_{a^{\prime}}-$ $\mathrm{L}_{a^{\prime}} \mathrm{L}_{a} \in \operatorname{End}_{\mathbb{C}}(\operatorname{Cliff}(g))$ where $\mathrm{L}_{a}$ and $\mathrm{L}_{a^{\prime}}$ are the (left) multiplications by $a$ and $a^{\prime}$ on $\operatorname{Cliff}(g)$ respectively. Set $\mathcal{D}_{\mathcal{W}, \mathcal{W}}:=\operatorname{span}_{\mathbb{C}}\left\{d_{w, w^{\prime}} \mid w, w^{\prime} \in \mathcal{W}\right\}$. Since for all $w, w^{\prime}, v, v^{\prime} \in \mathcal{W}, d_{w, w^{\prime}}$ stabilizes $\mathcal{W}$ and $d_{v, v^{\prime}} d_{w, w^{\prime}}-d_{w, w^{\prime}} d_{v, v^{\prime}}=$ $d_{d_{v, v^{\prime}} w, w^{\prime}}+d_{w, d_{v, v^{\prime}} w^{\prime}}$, we may consider $\mathcal{D}_{\mathcal{W}, \mathcal{W}}$ as a Lie subalgebra of the Lie algebra of the associative algebra $\operatorname{End}_{\mathbb{C}}(\mathcal{W})$.

Now suppose $u$ is defined as in (1.1) and consider $\operatorname{Cliff}(u)=\mathbb{C} 1 \oplus \mathcal{V}$. It is easy to check that $\mathcal{D}_{\mathcal{V}, \mathcal{V}}=\mathcal{G}$. So one can express the elements of $\mathcal{D}_{\mathcal{V}, \mathcal{V}}$ in matrix forms. More precisely for $1 \leq i, j \leq \ell$, we have

$$
\begin{array}{ccc}
d_{v_{i}, v_{\ell+j}} & =e_{\varepsilon_{i}-\varepsilon_{j}}, d_{v_{i}, v_{j}}=e_{\varepsilon_{i}+\varepsilon_{j}}, \quad d_{v_{\ell+i}, v_{\ell+j}}=-f_{\varepsilon_{i}+\varepsilon_{j}}, j>i \\
d_{v_{i}, v_{\ell+j}}=f_{\varepsilon_{j}-\varepsilon_{i}}, d_{v_{i}, v_{j}}=-e_{\varepsilon_{j}+\varepsilon_{i}}, d_{v_{\ell+i}, v_{\ell+j}}=f_{\varepsilon_{j}+\varepsilon_{i}}, & i>j, \\
d_{v_{i}, v_{\ell+j}}=H_{i}, \quad d_{v_{i}, v_{j}}=0, \quad d_{v_{\ell+i}, v_{\ell+j}}=0, & i=j, \\
d_{v_{2 \ell+1}, v_{i}}=e_{\varepsilon_{i}}, & d_{v_{\ell+i}, v_{2 \ell+1}}=\frac{1}{2} f_{\varepsilon_{i}} .
\end{array}
$$

Next, let $\Delta$ be an irreducible finite root system and $G$ be an abelian group. Denote by $X_{\Delta}$, a reduced finite root system of type $\Delta$ if $\Delta$ is reduced and of type $B, C$ or $D$ otherwise. Suppose $\mathfrak{g}$ is a finite dimensional simple Lie algebra over $\mathbb{C}$ with a Cartan subalgebra $\mathfrak{h}$ so that $\mathfrak{g}$ has a root space decomposition $\mathfrak{g}=\oplus_{\mu \in X_{\Delta}} \mathfrak{g}_{\mu}$ with $\mathfrak{h}=\mathfrak{g}_{0}$.

Definition 1.2. Let $\mathfrak{g}$ and $\mathfrak{h}$ be as above. A $\Delta$-graded Lie algebra $\mathcal{L}$ with grading pair $(\mathfrak{g}, \mathfrak{h})$ is a Lie algebra satisfying the following conditions:

(i) $\mathcal{L}$ contains $\mathfrak{g}$ as a subalgebra,

(ii) $\mathcal{L}=\oplus_{\mu \in \Delta} \mathcal{L}_{\mu}$, where $\mathcal{L}_{\mu}=\{x \in \mathcal{L} \mid[h, x]=\mu(h) x$ for all $h \in \mathfrak{h}\}$,

(iii) $\mathcal{L}_{0}=\sum_{\mu \in \Delta \times}\left[\mathcal{L}_{\mu}, \mathcal{L}_{-\mu}\right]$.

A $\Delta$-graded Lie algebra $\mathcal{L}$ with grading pair $(\mathfrak{g}, \mathfrak{h})$ is called $(\Delta, G)$-graded if $\mathcal{L}=\oplus_{g \in G} \mathcal{L}^{g}$ is a $G$-graded Lie algebra such that $\mathfrak{g} \subseteq \mathcal{L}^{0}$ and $\operatorname{supp}(\mathcal{L}):=\{g \in$ $\left.G \mid \mathcal{L}^{g} \neq\{0\}\right\}$ generates $G$. Since $\mathfrak{g} \subseteq \mathcal{L}^{0}, \mathcal{L}^{g}$ is an $\mathfrak{h}$-module for $g \in G$ and so we have $\mathcal{L}=\oplus_{\mu \in \Delta} \oplus_{g \in G} \mathcal{L}_{\mu}^{g}$ where $\mathcal{L}_{\mu}^{g}:=\mathcal{L}^{g} \cap \mathcal{L}_{\mu}$ for $g \in G$ and $\mu \in \Delta$ [MP, Proposition 2.1]. A $(\Delta, G)$-graded Lie algebra $\mathcal{L}$ is called a division $(\Delta, G)$-graded Lie algebra if for each $\mu \in \Delta^{\times}, g \in G$ and $0 \neq x \in \mathcal{L}_{\mu}^{g}$, there exists $y \in \mathcal{L}_{-\mu}^{-g}$ such that $[x, y]=t_{\mu}(\bmod Z(\mathcal{L}))$. A division $\left(\Delta, \mathbb{Z}^{\nu}\right)$-graded Lie algebra $\mathcal{L}$ with $\operatorname{dim}_{\mathbb{C}}\left(\mathcal{L}_{\mu}^{\sigma}\right) \leq 1$ for all $\sigma \in \mathbb{Z}^{\nu}$ and $\mu \in \Delta^{\times}$is called a Lie $\nu$-torus or simply a Lie torus.

Remark 1. It follows from [Yo1, Theorem 5.1] that if $\Delta$ is a finite root system of type $B_{\ell}$ and $\mathcal{L}=\oplus_{\mu \in \Delta} \oplus_{\sigma \in \mathbb{Z}^{\nu}} \mathcal{L}_{\mu}^{\sigma}$ is a $\left(\Delta, \mathbb{Z}^{\nu}\right)$-graded Lie algebra, 
then there exist semilattices (see [AABGP, Chapter II] for the terminology) $S, L \subseteq \mathbb{Z}^{\nu}$ such that $S=S_{\mu}$ for $\mu \in \Delta_{s h}$ and $L=S_{\mu}$ for $\mu \in \Delta_{l g}$ where for all $\mu \in \Delta, S_{\mu}:=\left\{\sigma \in \mathbb{Z}^{\nu} \mid \mathcal{L}_{\mu}^{\sigma} \neq\{0\}\right\}$. Let us call $(S, L)$ the corresponding pair of $\mathcal{L}$.

We recall that a central extension of a Lie algebra $\mathcal{L}$ is a pair $(\tilde{\mathcal{L}}, \pi)$ consisting of a Lie algebra $\tilde{\mathcal{L}}$ and an epimorphism $\pi: \tilde{\mathcal{L}} \longrightarrow \mathcal{L}$ whose kernel lies in the center of $\tilde{\mathcal{L}}$. A covering of $\mathcal{L}$ is a central extension $(\tilde{\mathcal{L}}, \pi)$ of $\mathcal{L}$ with $\tilde{\mathcal{L}}$ perfect $([\tilde{\mathcal{L}}, \tilde{\mathcal{L}}]=\tilde{\mathcal{L}})$. Any perfect Lie algebra $\mathcal{L}$ has a unique (up to isomorphism) universal central extension which is perfect called the universal covering algebra of $\mathcal{L}$ (see $[\mathrm{G}]$ ). Two perfect Lie algebras are said to be centrally isogenous if they have the same universal covering algebra.

Now let $\mathcal{G}$ be a finite dimensional simple Lie algebra of type $B_{\ell}, \ell \geq 3, \mathcal{V}$ be the short highest weight module of $\mathcal{G}$ and $u$ be defined as in (1.1).

Theorem 1.1 (Recognition Theorem for type $B_{\ell}$ ). ([BZ, Proposition 3.9 and Theorem 3.53]). Assume $A$ is a unital commutative associative algebra and $B$ is an $A$-module having a symmetric $A$-bilinear form $f: B \times B \longrightarrow A$. Then

$$
T(\operatorname{Cliff}(u) / \mathbb{C}, \operatorname{Cliff}(f) / A):=\left(\mathcal{G} \otimes_{\mathbb{C}} A\right) \oplus\left(\mathcal{V} \otimes_{\mathbb{C}} B\right) \oplus \mathcal{D}_{B, B}
$$

is a centerless $B_{\ell}$-graded Lie algebra whose Lie bracket is an extension of the Lie bracket on $\mathcal{D}_{B, B}$ satisfying

$$
\begin{array}{ll}
{[x \otimes a, v \otimes b]=x v \otimes a b,} & {\left[x \otimes a, x^{\prime} \otimes a^{\prime}\right]=\left[x, x^{\prime}\right] \otimes a a^{\prime},} \\
{[x \otimes a, D]=0,} & {\left[v \otimes b, v^{\prime} \otimes b^{\prime}\right]=d_{v, v^{\prime}} \otimes f\left(b, b^{\prime}\right)+u\left(v, v^{\prime}\right) d_{b, b^{\prime}},} \\
{[D, v \otimes b]=v \otimes D b,} &
\end{array}
$$

for $x, x^{\prime} \in \mathcal{G}, a, a^{\prime} \in A, v, v^{\prime} \in \mathcal{V}, b, b^{\prime} \in B$ and $D, D^{\prime} \in \mathcal{D}_{B, B}$. Conversely, Assume $\mathcal{L}$ is a Lie algebra which is $B_{\ell}$-graded for $\ell \geq 3$. Then there exist a unital commutative associative algebra $A$ and an $A$-module $B$ having a symmetric $A$-bilinear form $f$ such that $\mathcal{L}$ is centrally isogenous to $T(\operatorname{Cliff}(u) / \mathbb{C}, \operatorname{Cliff}(f) / A)$.

The following theorem states how to construct the universal covering algebra of a $B_{\ell}$-graded Lie algebra:

Theorem 1.2 ([ABG, Theorem 4.13]). By the same notation as in Theorem 1.1 consider the centerless $B_{\ell}$-graded Lie algebra $\Omega=(\mathcal{G} \otimes A) \oplus$ $(\mathcal{V} \otimes B) \oplus \mathcal{D}_{B, B}$. Set $\mathfrak{a}:=A \oplus B$, direct sum two vector spaces $A$ and $B$, and let $\mathfrak{s}$ be the subspace of $\mathfrak{a} \otimes \mathfrak{a}$ spanned by the elements

$$
\alpha \otimes \beta+\beta \otimes \alpha, \quad(\alpha \beta \otimes \gamma)+(\beta \gamma \otimes \alpha)+(\gamma \alpha \otimes \beta), \quad a \otimes b
$$


where $\alpha, \beta, \gamma \in \mathfrak{a}, a \in A$ and $b \in B$. Consider the quotient space

$$
\{\mathfrak{a}, \mathfrak{a}\}:=(\mathfrak{a} \otimes \mathfrak{a}) / \mathfrak{s}
$$

and for $\alpha, \beta \in \mathfrak{a}$, set $\{\alpha, \beta\}:=(\alpha \otimes \beta)+\mathfrak{s} \in\{\mathfrak{a}, \mathfrak{a}\}$. Let $\hat{\Omega}=(\mathcal{G} \otimes A) \oplus(\mathcal{V} \otimes$ $B) \oplus\{\mathfrak{a}, \mathfrak{a}\}$ and define a multiplication on $\hat{\Omega}$ by

$$
\begin{aligned}
& {\left[x \otimes a, x^{\prime} \otimes a^{\prime}\right]=\left[x, x^{\prime}\right] \otimes a a^{\prime}+\kappa\left(x, x^{\prime}\right)\left\{a, a^{\prime}\right\},} \\
& {[x \otimes a, v \otimes b]=x v \otimes a b=-[v \otimes b, x \otimes a],} \\
& {[x \otimes a,\{\alpha, \alpha\}]=0=-[\{\alpha, \alpha\}, x \otimes a],} \\
& {\left[v \otimes b, v^{\prime} \otimes b^{\prime}\right]=d_{v, v^{\prime}} \otimes f\left(b, b^{\prime}\right)+u\left(v, v^{\prime}\right)\left\{b, b^{\prime}\right\},} \\
& {\left[\left\{\alpha, \alpha^{\prime}\right\}, v \otimes b\right]=v \otimes d_{\alpha, \alpha^{\prime}} b=-\left[v \otimes b,\left\{\alpha, \alpha^{\prime}\right\}\right],} \\
& {\left[\left\{\alpha, \alpha^{\prime}\right\},\left\{\beta, \beta^{\prime}\right\}\right]=\left\{d_{\alpha, \alpha^{\prime}} \beta, \beta^{\prime}\right\}+\left\{\beta, d_{\alpha, \alpha^{\prime}} \beta^{\prime}\right\},}
\end{aligned}
$$

for $x, x^{\prime} \in \mathcal{G}, a, a^{\prime} \in A, v, v^{\prime} \in \mathcal{V}, b, b^{\prime} \in B$ and $\alpha, \alpha^{\prime}, \beta, \beta^{\prime} \in \mathfrak{a}$. Then $(\hat{\Omega}, \hat{\pi})$ where $\hat{\pi}: \hat{\Omega} \longrightarrow \Omega$ is given by $x \otimes a \mapsto x \otimes a ; u \otimes b \mapsto u \otimes b ;\left\{\alpha, \alpha^{\prime}\right\} \mapsto d_{\alpha, \alpha^{\prime}}$ is the universal covering algebra of $\Omega$.

Lemma 1.4. By the same notation as in Theorem 1.2, for $a, a^{\prime} \in A$ and $x, x^{\prime} \in \mathcal{G}$, we have the following:

(i) If $a^{\prime}=1$ or $\kappa\left(x, x^{\prime}\right)=0$, then $\left[x \otimes a, x^{\prime} \otimes a^{\prime}\right]=\left[x, x^{\prime}\right] \otimes a a^{\prime}$.

(ii) If $\left[x, x^{\prime}\right]=0$, then $\left[x \otimes a, x^{\prime} \otimes a^{\prime}, \omega\right]=\left[x^{\prime} \otimes a^{\prime}, x \otimes a, \omega\right]$ for all $\omega \in \hat{\Omega}$.

Proof. $\quad(i)$ Let $\alpha \in \mathfrak{a}$, by (1.11), $\{\alpha, 1\}=\{1, \alpha\}=0$. Using this together with (1.13), we are done.

(ii) Since $d_{a, a^{\prime}}(\alpha)=0$ for all $\alpha \in \mathfrak{a}$, (1.13) implies that $\{A, A\} \subseteq Z(\hat{\Omega})$. Now using the Jacobi identity, we are done.

Next let $\nu$ be a positive integer and take $A_{[\nu]}$ to be the algebra of Laurent polynomials in the commuting variables $t_{1}, \ldots, t_{\nu}$. For $\sigma=\left(n_{1}, \ldots, n_{\nu}\right) \in \mathbb{Z}^{\nu}$, by $t^{\sigma}$ we mean $t_{1}^{n_{1}} \ldots t_{\nu}^{n_{\nu}}$. Let $\mathcal{L}$ be a centerless $\nu$-torus over $\mathbb{C}$ of type $B_{\ell}$, $\ell \geq 3$. Then by [Yo2, Theorem 7.3] and [AG, Theorem 5.29] we may assume that the corresponding pair of $\mathcal{L}$ is $\left(S, 2 \mathbb{Z}^{\nu}\right)$ where $S=\biguplus_{j=0}^{m-1}\left(2 \mathbb{Z}^{\nu}+\tau_{j}\right)$ for some $m \geq 1$ and $\tau_{0}, \ldots, \tau_{m-1} \in \mathbb{Z}^{\nu}$ satisfying $\tau_{0}=0$ and $\tau_{r} \not \equiv \tau_{s}\left(\bmod 2 \mathbb{Z}^{\nu}\right)$ for $0 \leq s \neq r \leq m-1$. Furthermore

$$
\mathcal{L} \cong T\left(\operatorname{Cliff}(u) / \mathbb{C}, \operatorname{Cliff}(g) / A_{[\nu]}\right)
$$

where $u$ is defined as in (1.1) and $g$ is the symmetric $A_{[\nu]}$-bilinear form on $A_{[\nu]}^{m-1}, m-1$ copies of $A_{[\nu]}$, defined as follows: 
$(1.15)$

$$
g: A_{[\nu]}^{m-1} \times A_{[\nu]}^{m-1} \longrightarrow A_{[\nu]}^{m-1} ; \quad\left(\sum_{r=1}^{m-1} a_{r} w_{r}, \sum_{r=1}^{m-1} b_{r} w_{r}\right) \longmapsto \sum_{r=1}^{m-1} a_{r} b_{r} t^{\tau_{r}}
$$

in which $\left\{w_{1}, \ldots, w_{m-1}\right\}$ is the standard basis for $A_{[\nu]}^{m-1}$ over $A_{[\nu]}$. One can use Theorem 1.2 to conclude that the universal covering algebra of $\mathcal{L}$ is $\mathfrak{A}=$ $\left(\mathcal{G} \otimes_{\mathbb{C}} A_{[\nu]}\right) \oplus\left(\mathcal{V} \otimes_{\mathbb{C}} A_{[\nu]}^{m-1}\right) \oplus\{\mathfrak{a}, \mathfrak{a}\}$ where $\mathfrak{a}=A_{[\nu]} \oplus A_{[\nu]}^{m-1}$ and $\{\mathfrak{a}, \mathfrak{a}\}$ is defined as in (1.12). So Theorem 1.1 implies that

$$
\mathfrak{A} / Z(\mathfrak{A})=\left(\mathcal{G} \otimes_{\mathbb{C}} A_{[\nu]}\right) \oplus\left(\mathcal{V} \otimes_{\mathbb{C}} A_{[\nu]}^{m-1}\right) \oplus \mathcal{D}_{A_{[\nu]}^{m-1}, A_{[\nu]}^{m-1}} .
$$

It follows from (1.13) together with Lemma 1.4 that $\mathfrak{A}$ is generated by

$$
\begin{gathered}
e_{i} \otimes 1, f_{i} \otimes 1, h_{i} \otimes 1, h_{i} \otimes t_{j}, h_{i} \otimes t_{j}^{-1}, v_{1} \otimes w_{s}, \\
1 \leq i \leq \ell, 1 \leq j \leq \nu, 1 \leq s \leq m-1 .
\end{gathered}
$$

Moreover using (1.13), Lemma 1.4 and (1.9), for $1 \leq i, r \leq \ell, 1 \leq j \leq \nu$ and $1 \leq s, t \leq m-1$, the following relations are satisfied in $\mathfrak{A}$ :

$$
\begin{aligned}
& {\left[\alpha\left(h_{r}\right)\left(h_{i} \otimes t_{j}^{ \pm 1}\right)-\alpha\left(h_{i}\right)\left(h_{r} \otimes t_{j}^{ \pm 1}\right), x_{\alpha} \otimes 1\right]=0 ; \alpha \in \Phi, x_{\alpha} \in \mathcal{G}_{\alpha},} \\
& {\left[\beta\left(h_{r}\right)\left(h_{i} \otimes t_{j}^{ \pm 1}\right)-\beta\left(h_{i}\right)\left(h_{r} \otimes t_{j}^{ \pm 1}\right), v_{\beta} \otimes w_{s}\right]=0 ; \beta \in \Phi_{s h}, v_{\beta} \in \mathcal{V}_{\beta},}
\end{aligned}
$$

to which we refer as quasi-diagonal relations, also

$$
\begin{aligned}
& {\left[h_{r} \otimes t_{j}, h_{i} \otimes t_{j}^{-1}, x \otimes 1\right]=\alpha\left(h_{i}\right) \alpha\left(h_{r}\right) x \otimes 1 ; \quad x \in \mathcal{G}_{\alpha}, \alpha \in \Phi,} \\
& {\left[h_{r} \otimes t_{j}, h_{i} \otimes t_{j}^{-1}, y \otimes w_{s}\right]=\alpha\left(h_{i}\right) \alpha\left(h_{r}\right) y \otimes w_{s} ; y \in \mathcal{V}_{\alpha}, \alpha \in \Phi_{s h},}
\end{aligned}
$$

that we call cancelling relations and

$$
\begin{gathered}
{\left[v_{1} \otimes w_{s}, v_{1} \otimes w_{t}\right]=0, \quad\left[v_{1} \otimes w_{s}, v_{i} \otimes w_{t}\right]=\delta_{s, t} e_{\varepsilon_{1}+\varepsilon_{i}} \otimes t^{\tau_{s}} ; \quad i \geq 2,} \\
{\left[v_{1} \otimes w_{s}, v_{\ell+i} \otimes w_{t}\right]=\delta_{s, t} e_{\varepsilon_{1}-\varepsilon_{i}} \otimes t^{\tau_{s}} ; \quad i \geq 3,}
\end{gathered}
$$

which we call basic short part relations.

\section{$\S 2 . \quad$ Presentation}

Throughout this section $\nu, \ell$ and $m$ are positive integers so that $\ell \geq 3 . \mathcal{G}$ and $\mathcal{V}$ are as in $\S 1$ and $u$ is the form on $\mathcal{V}$ defined by (1.1). We find a finite presentation of the universal covering algebra of a Lie torus of type $B_{\ell}$. More precisely, we consider a set $\left\{\tau_{r} \mid 0 \leq r \leq m-1\right\}$ of some representatives of cosets of $2 \mathbb{Z}^{\nu}$ in $\mathbb{Z}^{\nu}$ with $\tau_{0}=0$ and find a finite presentation of the universal covering 
algebra of the Lie torus of type $B_{\ell}$ whose corresponding pair is $\left(S, 2 \mathbb{Z}^{\nu}\right)$ with $S=\uplus_{r=0}^{m-1}\left(\tau_{r}+2 \mathbb{Z}^{\nu}\right)$. We postpone the case that $S=2 \mathbb{Z}^{\nu}$ (corresponding to $m=$ 1) to Remark 3. So we suppose that $m \geq 2$ and fix $\tau_{r}=\left(n_{1}^{r}, n_{2}^{r}, \ldots, n_{\nu}^{r}\right) \in \mathbb{Z}^{\nu}$, $1 \leq r \leq m-1$, such that $\tau_{1}+2 \mathbb{Z}^{\nu}, \ldots, \tau_{m-1}+2 \mathbb{Z}^{\nu}$ are disjoint nonzero cosets of $2 \mathbb{Z}^{\nu}$ in $\mathbb{Z}^{\nu}$. We represent a finite presentation of the universal covering algebra $\mathfrak{A}$ of $T\left(\operatorname{Cliff}(u) / \mathbb{C}, \operatorname{Cliff}(g) / A_{[\nu]}\right)$ where $g$ is the symmetric $A_{[\nu]}$-bilinear form on $A_{[\nu]}^{m-1}$ defined by (1.15). We first construct a finitely presented Lie algebra $\mathcal{L}$ consisting of several generators and a bunch of relations. We decompose $\mathcal{L}$ into irreducible $\mathcal{G}$-modules isomorphic to $\mathcal{G}$, irreducible $\mathcal{G}$-modules isomorphic to $\mathcal{V}$ and trivial $\mathcal{G}$-modules. Next we prove that $\mathcal{L}$ is a $B_{\ell}$-graded Lie algebra that is a central extension of $\mathfrak{A}$. Then we have enough tools to prove that $\mathcal{L} \cong \mathfrak{A}$. We collect the relations which have similar natures in the same collection. To begin, we consider the Cartan matrix $C=\left(c_{i, j}\right)_{i, j}$ of type $B_{\ell}$ and take $\mathcal{L}_{s}$ to be the Lie algebra generated by $3 \ell+2 \ell \nu+m-1$ elements

$$
\left\{e_{i}, f_{i}, h_{i}, h_{i, j}^{ \pm}, v^{r} \mid 1 \leq i \leq \ell, 1 \leq j \leq \nu, 1 \leq r \leq m-1\right\}
$$

subject to the following type of relations:

Serre's relations:

(R1) $\left[h_{i}, h_{j}\right]=0,\left[e_{i}, f_{j}\right]=\delta_{i, j} h_{i},\left[h_{i}, e_{j}\right]=c_{j, i} e_{j},\left[h_{i}, f_{j}\right]=-c_{j, i} f_{j}$, $\left(\operatorname{ad} e_{i}\right)^{-c_{j, i}+1}\left(e_{j}\right)=0,\left(\operatorname{ad} f_{i}\right)^{-c_{j, i}+1}\left(f_{j}\right)=0,1 \leq i, j \leq \ell$.

Short highest weight module relations:

(R2) $\left[e_{i}, v^{r}\right]=0,\left[h_{i}, v^{r}\right]=\delta_{1, i} v^{r},\left[f_{j}, v^{r}\right]=\left[f_{1}, f_{1}, v^{r}\right]=0$,

$$
1 \leq i \leq \ell, 2 \leq j \leq \ell, 1 \leq r \leq m-1 \text {. }
$$

Now let $\mathcal{G}$ be the subalgebra of $\mathcal{L}_{s}$ generated by $\left\{e_{i}, f_{i}, h_{i}\right\}_{i=1}^{\ell}$. Since $\left\{e_{i}, f_{i}, h_{i}\right\}_{i=1}^{\ell}$ satisfies Serre's relations, $\mathcal{G}$ is a finite dimensional simple Lie algebra of type $B_{\ell}$ [H, Theorem 18.3]. So $\mathcal{G}=\oplus_{\alpha \in \Phi} \mathcal{G}_{\alpha}$ where $\Phi=\{0\} \cup$ $\left\{ \pm \varepsilon_{i}, \pm\left(\varepsilon_{i} \pm \varepsilon_{j}\right) \mid 1 \leq i \neq j \leq \ell\right\}$ and where $\varepsilon_{i}\left(h_{j}\right)=\delta_{i, j}-\delta_{i, j+1}, 1 \leq j \leq \ell-1$, and $\varepsilon_{i}\left(h_{\ell}\right)=2 \delta_{i, \ell}$. Moreover for each $\alpha \in \Phi^{+}$, there exist $e_{\alpha} \in \mathcal{G}_{\alpha}$ and $f_{\alpha} \in \mathcal{G}_{-\alpha}$ such that $\left(e_{\alpha},\left[e_{\alpha}, f_{\alpha}\right], f_{\alpha}\right)$ is an $\mathfrak{s l}_{2}-$ triple. Without loose of generality we may assume $e_{\alpha}$ and $f_{\alpha}\left(\alpha \in \Phi^{+}\right)$are defined as in (1.6). Now for $1 \leq j \leq \nu$, define

$$
k_{j}^{ \pm}:=h_{1, j}^{ \pm}+\sum_{t=2}^{\ell-1} 2 h_{t, j}^{ \pm}+h_{\ell, j}^{ \pm}
$$


and set

$$
\begin{aligned}
S_{g} & :=\operatorname{span}_{\mathbb{C}}\left\{e_{i}, f_{i}, h_{i}, h_{i, j}^{ \pm}, v^{r} \mid 1 \leq i \leq \ell, 1 \leq j \leq \nu, 1 \leq r \leq m-1\right\} . \\
S_{2} & :=\operatorname{span}_{\mathbb{C}}\left\{e_{i}, f_{i} \mid 1 \leq i \leq \ell, i \neq 2\right\} . \\
H & :=\operatorname{span}_{\mathbb{C}}\left\{h_{i} \mid 1 \leq i \leq \ell\right\} . \\
\mathfrak{h} & :=\operatorname{span}_{\mathbb{C}}\left\{h_{i, j}^{ \pm} \mid 1 \leq i \leq \ell, 1 \leq j \leq \nu\right\} . \\
\mathfrak{h}_{2} & :=\operatorname{span}_{\mathbb{C}}\left\{h_{i, j}^{ \pm} \mid 1 \leq i \leq \ell, i \neq 2,1 \leq j \leq \nu\right\} . \\
\mathfrak{h}_{\theta} & :=\operatorname{span}_{\mathbb{C}}\left\{k_{j}^{ \pm} \mid 1 \leq j \leq \nu\right\} . \\
Z_{h} & :=\operatorname{span}_{\mathbb{C}}\left\{\left[h_{i, j}^{ \pm}, h_{r, s}^{ \pm}\right] \mid 1 \leq i, r \leq \ell, 1 \leq j, s \leq \nu\right\} . \\
\mathfrak{h}_{\alpha}^{i, t} & :=\operatorname{span}_{\mathbb{C}}\left\{\alpha\left(h_{t}\right) h_{i, j}^{-}-\alpha\left(h_{i}\right) h_{t, j}^{-}, \alpha\left(h_{t}\right) h_{i, j}^{+}-\alpha\left(h_{i}\right) h_{t, j}^{+} \mid 1 \leq j \leq \nu\right\} \\
& \alpha \in \Phi, 1 \leq i, t \leq \ell .
\end{aligned}
$$

Since $\mathcal{G}$ is a subalgebra of $\mathcal{L}_{s}, \mathcal{L}_{s}$ is a $\mathcal{G}$-module. Now for $1 \leq r \leq m-1$, define $\mathcal{V}^{r}$ to be the $\mathcal{G}$-submodule of $\mathcal{L}_{s}$ generated by $v^{r}$. Using (R2), one can see that $\mathcal{V}^{r}$ is a finite dimensional irreducible $\mathcal{G}$-module of highest weight $\varepsilon_{1}$ [H, Theorem 21.4]. Contemplating (1.2), (1.3) and using (1.7)(i) together with Lemmas 1.2 and 1.3, we may assume $\mathcal{V}^{r}$ admits a weight space decomposition relative to $H$ as follows:

$$
\begin{gathered}
\mathcal{V}^{r}=\sum_{\alpha \in\left\{ \pm \varepsilon_{i}\right\}_{i=1}^{\ell} \cup\{0\}}\left(\mathcal{V}^{r}\right)_{\alpha} \text { with } \\
\left(\mathcal{V}^{r}\right)_{0}=\mathbb{C} v_{2 \ell+1}^{r},\left(\mathcal{V}^{r}\right)_{\varepsilon_{i}}=\mathbb{C} v_{i}^{r},\left(\mathcal{V}^{r}\right)_{-\varepsilon_{i}}=\mathbb{C} v_{\ell+i}^{r} \text { where } \\
v_{1}^{r}=v^{r}, v_{i}^{r}=\left[f_{i-1}, \ldots, f_{1}, v^{r}\right]=\left[\left[f_{i-1}, \ldots, f_{1}\right], v^{r}\right] ; 2 \leq i \leq \ell, \\
v_{2 \ell+1}^{r}=-\frac{1}{2}\left[f_{\ell}, \ldots, f_{1}, v^{r}\right]=-\frac{1}{2}\left[f, v^{r}\right] \text { where } f=\left[f_{\ell}, \ldots, f_{1}\right], \\
v_{\ell+i}^{r}=\frac{(-1)^{\ell-i}}{2}\left[f_{i}, \ldots, f_{\ell}, v_{2 \ell+1}^{r}\right]=\frac{-(-1)^{\ell-i}}{4}\left\{\begin{array}{l}
{\left[f_{1},\left[f_{2}, \ldots, f_{\ell}, f\right], v^{r}\right] \quad i=1,} \\
{\left[\left[f_{i}, \ldots, f_{\ell}, f\right], v^{r}\right] \quad 2 \leq i \leq \ell .}
\end{array}\right.
\end{gathered}
$$

Now let $\mathcal{L}_{g}$ be the Lie algebra $\mathcal{L}_{s}$ modulo the ideal generating the following relations:

(R3) $(i)\left[Z_{h}, S_{g}\right]=0 \quad\left(Z_{h}\right.$ is central $), \quad(i i)[H, \mathfrak{h}]=0$.

(R4)

Quasi-diagonal relations:
(i) $\left[\mathfrak{h}_{2}, e_{\varepsilon_{1}+\varepsilon_{2}}\right]=0$
(ii) $\left[\mathfrak{h}_{\theta}, S_{2}\right]=0, \quad(i i i)\left[\mathfrak{h}_{\varepsilon_{2}-\varepsilon_{3}}^{2,3}, \mathbb{C} f_{2}+\mathbb{C} e_{2}\right]=0$. 
Quasi-diagonal relations:

(R5) $\begin{aligned} & (i)\left[\mathfrak{h}, v_{2 \ell+1}^{r}\right]=0, \\ & (i i)\left[\mathfrak{h}_{\alpha}^{i, t},\left(\mathcal{V}^{r}\right)_{\alpha}\right]=0,\end{aligned}, \alpha \in \Phi_{s h}, 1 \leq i, t \leq \ell, 1 \leq r \leq m-1$ (see (2.3)).

Cancelling relations:

(R6) ${ }^{(i)}\left[h_{s, j}^{-}, h_{i, j}^{+}, x\right]=\alpha\left(h_{i}\right) \alpha\left(h_{s}\right) x, x \in \mathcal{G}_{\alpha}, \alpha \in \Phi$,

(ii) $\left[h_{s, j}^{-}, h_{i, j}^{+}, y\right]=\alpha\left(h_{i}\right) \alpha\left(h_{s}\right) y, y \in\left(\mathcal{V}^{r}\right)_{\alpha}, \alpha \in \Phi_{s h}$,

$1 \leq j \leq \nu, 1 \leq i, s \leq \ell, 1 \leq r \leq m-1$.

Basic short part relations:

(i) $\left[v^{r}, v^{t}\right]=0,1 \leq r, t \leq m-1$,

(R7) (ii) $\left[v^{r}, v_{i}^{t}\right]=\delta_{r, t}\left(\operatorname{ad} a_{1}^{i, t}\right)^{\left|n_{1}^{t}\right|} \ldots\left(\operatorname{ad} a_{\nu}^{i, t}\right)^{\left|n_{\nu}^{t}\right|} e_{\varepsilon_{1}+\varepsilon_{i}}, \quad 2 \leq i \leq \ell$,

(iii) $\left[v^{r}, v_{\ell+i}^{t}\right]=\delta_{r, t}\left(\operatorname{ad} a_{1}^{i, t}\right)^{\left|n_{1}^{t}\right|} \ldots\left(\operatorname{ad} a_{\nu}^{i, t}\right)^{\left|n_{\nu}^{t}\right|} e_{\varepsilon_{1}-\varepsilon_{i}}, \quad 3 \leq i \leq \ell$,

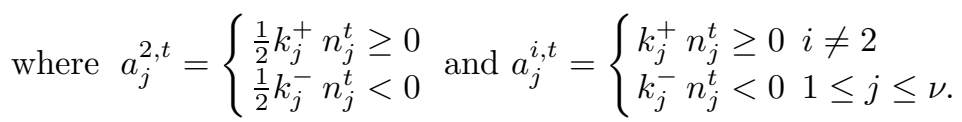

Now for $1 \leq j \leq \nu$ set

$$
\begin{aligned}
& e_{1, j}^{ \pm}:=-\frac{1}{8}\left[f_{\varepsilon_{2}}, f_{\varepsilon_{2}}, k_{j}^{ \pm}, e_{\varepsilon_{1}+\varepsilon_{2}}\right], \quad f_{1, j}^{ \pm}:=\frac{1}{8}\left[f_{\varepsilon_{1}}, f_{\varepsilon_{1}}, k_{j}^{ \pm}, e_{\varepsilon_{1}+\varepsilon_{2}}\right], \\
& e_{\ell, j}^{ \pm}:=\frac{1}{4}\left[f_{\varepsilon_{2}}, f_{\varepsilon_{1}-\varepsilon_{\ell}}, k_{j}^{ \pm}, e_{\varepsilon_{1}+\varepsilon_{2}}\right], \quad f_{\ell, j}^{ \pm}:=-\frac{1}{8}\left[f_{\varepsilon_{1}}, f_{\ell}, f_{\varepsilon_{2}}, k_{j}^{ \pm}, e_{\varepsilon_{1}+\varepsilon_{2}}\right], \\
& e_{2, j}^{ \pm}:=\frac{1}{8}\left[f_{\varepsilon_{3}}, f_{\varepsilon_{1}}, k_{j}^{ \pm}, e_{\varepsilon_{1}+\varepsilon_{2}}\right], \\
& e_{i, j}^{ \pm}:=-\frac{1}{8}\left[f_{\varepsilon_{i+1}}, f_{\varepsilon_{2}}, f_{\varepsilon_{1}-\varepsilon_{i}}, k_{j}^{ \pm}, e_{\varepsilon_{1}+\varepsilon_{2}}\right], \quad 3 \leq i \leq \ell-1, \\
& f_{i, j}^{ \pm}:=-\frac{1}{8}\left[f_{\varepsilon_{i}}, f_{\varepsilon_{2}}, f_{\varepsilon_{1}-\varepsilon_{i+1}}, k_{j}^{ \pm}, e_{\varepsilon_{1}+\varepsilon_{2}}\right], \quad 2 \leq i \leq \ell-1,
\end{aligned}
$$

and define

$$
\begin{aligned}
& \mathcal{E}:=\operatorname{span}_{\mathbb{C}}\left\{e_{i, j}^{ \pm} \mid 1 \leq i \leq \ell, 1 \leq j \leq \nu\right\}, \\
& \mathcal{F}:=\operatorname{span}_{\mathbb{C}}\left\{f_{i, j}^{ \pm} \mid 1 \leq i \leq \ell, 1 \leq j \leq \nu\right\} .
\end{aligned}
$$

Finally define $\mathcal{L}$ to be the Lie algebra $\mathcal{L}_{g}$ modulo the ideal generating the following relations:

(R8) $\left[e_{i, j}^{ \pm}, f_{i}\right]=h_{i j}^{ \pm} ; 1 \leq i \leq \ell, 1 \leq j \leq \nu$.

(R9) $[\mathfrak{h}, \mathfrak{h}, \mathcal{E}, \mathcal{F}]=0,\left[\sum_{i=1}^{\ell} \mathbb{C} f_{i}, \mathfrak{h}, \mathcal{E}, \mathcal{F}\right]=0$.

Theorem 2.1 (Main Theorem). $\quad \mathcal{L}$ is the universal covering algebra of $T\left(\operatorname{Cliff}(u) / \mathbb{C}, \operatorname{Cliff}(g) / A_{[\nu]}\right)$, in other words $\mathcal{L} \cong \mathfrak{A}$. 
To prove this theorem, we need to know the structure of $\mathcal{L}$. To begin, we recall that $\mathfrak{A}$ is generated by the elements stated in (1.17). It follows using (1.13) that this generating set satisfies the relations (R1)-(R9). So there exists a Lie algebra epimorphism from $\mathcal{L}$ to $\mathfrak{A}$ as follows:

$$
\begin{gathered}
c \psi: \mathcal{L} \longrightarrow \mathfrak{A} \\
e_{i} \mapsto e_{i} \otimes 1, f_{i} \mapsto f_{i} \otimes 1, h_{i} \mapsto h_{i} \otimes 1, h_{i, j}^{ \pm} \mapsto h_{i} \otimes t_{j}^{ \pm 1}, v^{r} \mapsto v_{1} \otimes w_{r}, \\
1 \leq i \leq \ell, 1 \leq j \leq \nu, 1 \leq r \leq m-1 .
\end{gathered}
$$

Lemma 2.1. Let $a, b \in \mathfrak{h}$ and $x \in \mathcal{L}$, then $[a, b, x]=[b, a, x]$.

Proof. By $(\mathrm{R} 3)(i)$, we have $[a, b] \subseteq Z(\mathcal{L})$. Using this together with the Jacobi identity, we are done.

We know that $\mathcal{G}$ is a subalgebra of $\mathcal{L}$. Another point about the structure of $\mathcal{L}$ is that, under the adjoint action of $\mathcal{G}$ on $\mathcal{L}, \mathcal{L}$ decomposes into a direct sum of

- modules isomorphic to the adjoint module $\mathcal{G}$.

- modules isomorphic to $\mathcal{V}$.

- one-dimensional $\mathcal{G}$-modules.

We show this point in some steps. So we arrange the rest of this section as follows. The first three subsections are respectively devoted to introducing some irreducible $\mathcal{G}$-submodules of $\mathcal{L}$ isomorphic to $\mathcal{G}$, irreducible $\mathcal{G}$-submodules of $\mathcal{L}$ isomorphic to $\mathcal{V}$ and trivial $\mathcal{G}$-submodules. We study the properties of the introduced $\mathcal{G}$-submodules in each subsection. Subsection 4 deals with the relations between the introduced $\mathcal{G}$-submodules. In Subsection 5 , we get familiar with some central elements of $\mathcal{L}$. Finally in the last subsection we get the mentioned decomposition and use it to prove our main theorem.

\section{$\S 2.1 . \quad \mathcal{G}$-submodules isomorphic to $\mathcal{G}$}

Definition 2.1. Let $\sigma=\left(n_{1}, \ldots, n_{\nu}\right) \in \mathbb{Z}^{\nu}$. We call $|\sigma|:=\sum_{s=1}^{\nu}\left|n_{s}\right|$ the norm of $\sigma$. For $\sigma \neq 0$, if $1 \leq j \leq \nu$ and $1+\sum_{s=1}^{j-1}\left|n_{s}\right| \leq i \leq \sum_{s=1}^{j}\left|n_{s}\right|$, define $a_{i}:=\left\{\begin{array}{l}k_{j}^{+} \text {if } n_{j}>0 \\ k_{j}^{-} \text {if } n_{j}<0\end{array}(\right.$ see $(2.2))$ and set $a_{\sigma}:=\left(a_{1}, \ldots, a_{|\sigma|}\right)$. Also set $a_{0}:=\left(k_{1}^{-}, k_{1}^{+}\right)$. We call $a_{\sigma}$ the norm-tuple of $\sigma$. 
Now let $\sigma \in \mathbb{Z}^{\nu}$ and define

$e_{\sigma}:=\left[\frac{1}{2} a_{1}, \ldots, \frac{1}{2} a_{t}, e_{\theta}\right]$ where $\left(a_{1}, \ldots, a_{t}\right)$ is the norm-tuple of $\sigma$ and $e_{\theta}=e_{\varepsilon_{1}+\varepsilon_{2}}$ is a maximal vector of highest weight $\theta=\varepsilon_{1}+\varepsilon_{2}$ in $\mathcal{G}$-module $\mathcal{G}$. Using $(\mathrm{R} 6)(i)$, we have $e_{0}=e_{\theta}$.

Set

$$
\mathcal{G}_{\sigma}:=\mathcal{G} \text {-submodule of } \mathcal{L} \text { generated by } e_{\sigma} ; \sigma \in \mathbb{Z}^{\nu}
$$

Proposition 2.1. $\mathcal{G}_{\sigma}, \sigma \in \mathbb{Z}^{\nu}$, is an irreducible finite dimensional $\mathcal{G}-$ module of highest weight $\theta$. In fact as $\mathcal{G}$-modules, $\mathcal{G}_{\sigma} \simeq \mathcal{G}$.

Proof. Contemplating (2.5), one can see that $\psi\left(e_{\sigma}\right)=e_{\theta} \otimes t^{\sigma} \neq 0$. Therefore $e_{\sigma} \neq 0$. Thus by [H, Theorem 21.4], it is enough to show

$$
\begin{aligned}
{\left[h_{i}, e_{\sigma}\right]=} & \theta\left(h_{i}\right) e_{\sigma}, \quad\left[e_{i}, e_{\sigma}\right]=0, \quad[\overbrace{f_{i}, f_{i}, \ldots, f_{i}}^{m_{i}+1}, e_{\sigma}]=0 \\
& \text { where } 1 \leq i \leq \ell \text { and } m_{i}=\theta\left(h_{i}\right) .
\end{aligned}
$$

Fix $1 \leq i \leq \ell$. We first mention that the equalities in (2.8) hold for $\sigma=0$ as $e_{\theta}$ is a maximal vector in $\mathcal{G}$-module $\mathcal{G}$. Now let $0 \neq \sigma \in \mathbb{Z}^{\nu}$ and $e_{\sigma}=\left[a_{1}, \ldots, a_{s}, e_{\theta}\right]$ for some $s \in \mathbb{N}$. Then $(\mathrm{R} 3)(i i)$ implies that $\left[h_{i}, e_{\sigma}\right]=\left[a_{1}, \ldots, a_{s}, h_{i}, e_{\theta}\right]=$ $\theta\left(h_{i}\right) e_{\sigma}$. Also if $i \neq 2$, then by $(\mathrm{R} 4)(i i)$ we have

$$
[\underbrace{f_{i}, f_{i}, \ldots, f_{i}}_{m_{i}+1}, e_{\sigma}]=[a_{1}, \ldots, a_{s}, \underbrace{f_{i}, f_{i}, \ldots, f_{i}}_{m_{i}+1}, e_{\theta}]=\left[a_{1}, \ldots, a_{s}, 0\right]=0 .
$$

Next we use induction on $|\sigma|$ to prove $\left[e_{i}, e_{\sigma}\right]=0$ and $\left[f_{2}, f_{2}, e_{\sigma}\right]=0$. Let $\sigma, \tau \in \mathbb{Z}^{\nu}$ such that $e_{\sigma}=\left[a_{1}, \ldots, a_{s}, e_{\theta}\right], e_{\tau}=\left[a_{0}, a_{1}, \ldots, a_{s}, e_{\theta}\right],\left[e_{i}, e_{\sigma}\right]=0$ and $\left[f_{2}, f_{2}, e_{\sigma}\right]=0$. If $i \neq 2$, then by (R4)(ii) and the induction hypothesis, we have $\left[e_{i}, e_{\tau}\right]=\left[a_{0}, e_{i}, e_{\sigma}\right]=0$. Now let $i=2$ and $a_{0}=\frac{1}{2} k_{j}^{ \pm}$for some $1 \leq j \leq \nu$. It follows using $(\mathrm{R} 4)(i i)$ that

$$
\left[\frac{1}{2} k_{j}^{ \pm}, e_{\theta}\right]=\left[h_{2, j}^{ \pm}+2 h_{3, j}^{ \pm}, e_{\theta}\right]
$$

This together with Lemma 2.1 implies that

$$
e_{\tau}=\left[a_{0}, a_{1}, \ldots, a_{s}, e_{\theta}\right]=\left[a_{1}, \ldots, a_{s}, h_{2, j}^{ \pm}+2 h_{3, j}^{ \pm}, e_{\theta}\right]=\left[h_{2, j}^{ \pm}+2 h_{3, j}^{ \pm}, e_{\sigma}\right] .
$$

Using this together with $(\mathrm{R} 4)(\mathrm{iii})$ and the induction hypothesis, we have

$$
\left[e_{2}, e_{\tau}\right]=\left[e_{2}, h_{2, j}^{ \pm}+2 h_{3, j}^{ \pm}, e_{\sigma}\right]=\left[h_{2, j}^{ \pm}+2 h_{3, j}^{ \pm}, e_{2}, e_{\sigma}\right]=0
$$


and

$$
\left[f_{2}, f_{2}, e_{\tau}\right]=\left[f_{2}, f_{2}, h_{2, j}^{ \pm}+2 h_{3, j}^{ \pm}, e_{\sigma}\right]=\left[h_{2, j}^{ \pm}+2 h_{3, j}^{ \pm}, f_{2}, f_{2}, e_{\sigma}\right]=0
$$

This completes the proof.

Now let $\sigma \in \mathbb{Z}^{\nu}$. Using Proposition 2.1, one concludes that there exists a $\mathcal{G}$-module isomorphism $\varphi_{\sigma}: \mathcal{G} \longrightarrow \mathcal{G}_{\sigma}$ mapping $e_{\theta}$ to $e_{\sigma}$. So

$$
\begin{aligned}
& \text { if } x, y, z, w \in \mathcal{G} \text { such that }[x, y]=[z, w] \\
& \text { then } \varphi_{\sigma}([x, y])=\left[x, \varphi_{\sigma}(y)\right]=\left[z, \varphi_{\sigma}(w)\right] \text {. }
\end{aligned}
$$

Also $\mathcal{G}_{\sigma}$ admits a weight space decomposition $\mathcal{G}_{\sigma}=\sum_{\alpha \in \Phi}\left(\mathcal{G}_{\sigma}\right)_{\alpha}$ where $\left(\mathcal{G}_{\sigma}\right)_{\alpha}=$ $\varphi_{\sigma}\left(\mathcal{G}_{\alpha}\right), \alpha \in \Phi$. Consider the base $\left\{\alpha_{i}\right\}_{i=1}^{\ell}$ of $\Phi$ defined in $\S 1$, then we have

$$
\left(\mathcal{G}_{\sigma}\right)_{0}=\varphi_{\sigma}\left(\mathcal{G}_{0}\right)=\sum_{i=1}^{\ell} \mathbb{C} \varphi_{\sigma}\left(h_{i}\right)=\sum_{i=1}^{\ell} \varphi_{\sigma}\left(\left[f_{i}, \mathcal{G}_{\alpha_{i}}\right]\right)=\sum_{i=1}^{\ell}\left[f_{i},\left(\mathcal{G}_{\sigma}\right)_{\alpha_{i}}\right]
$$

Next let $1 \leq i \leq \ell$ and set

$$
\begin{aligned}
& e_{i, \sigma}:=\varphi_{\sigma}\left(e_{i}\right), f_{i, \sigma}:=\varphi_{\sigma}\left(f_{i}\right), h_{i, \sigma}:=\varphi_{\sigma}\left(h_{i}\right), \\
& H_{i, \sigma}:=\varphi_{\sigma}\left(H_{i}\right) \text { where } H_{i}=\sum_{r=i}^{\ell-1} h_{r}+(1 / 2) h_{\ell} .
\end{aligned}
$$

Then (2.10) implies that

(i) $\left[e_{i}, e_{i, \sigma}\right]=\left[f_{i}, f_{i, \sigma}\right]=0$,

(ii) $\left[e_{i}, f_{j, \sigma}\right]=-\left[f_{j}, e_{i, \sigma}\right]=\delta_{i, j} h_{i, \sigma}$,

(iii) $\left[h_{i}, e_{i, \sigma}\right]=-\left[e_{i}, h_{i, \sigma}\right]=2 e_{i, \sigma} \quad$ and $\quad\left[h_{i}, f_{i, \sigma}\right]=-\left[f_{i}, h_{i, \sigma}\right]=-2 f_{i, \sigma}$,

$(i v)\left[e_{j}, h_{i, \sigma}\right]=\left(\alpha_{j}\left(h_{i}\right) / 2\right)\left[e_{j}, h_{j, \sigma}\right]$ and $\left[f_{j}, h_{i, \sigma}\right]=\left(\alpha_{j}\left(h_{i}\right) / 2\right)\left[f_{j}, h_{j, \sigma}\right]$

where $1 \leq i, j \leq \ell$ and $\sigma \in \mathbb{Z}^{\nu}$. Now set

$$
\sigma_{j}^{ \pm}:=(0, \ldots, 0, \underbrace{ \pm 1}_{j \mathrm{th}}, 0, \ldots, 0), \quad \varphi_{j}^{ \pm}:=\varphi_{\sigma_{j}^{ \pm}} ; 1 \leq j \leq \nu .
$$

One knows that $e_{\sigma_{j}^{ \pm}}=\left[(1 / 2) k_{j}^{ \pm}, e_{\theta}\right]$, so using (2.4) together with $(2.13)(i i)$ and (R8), for $1 \leq i \leq \ell$ and $1 \leq j \leq \nu$, we have
(i) $e_{i, j}^{ \pm}=\varphi_{j}^{ \pm}\left(e_{i}\right)$
(ii) $f_{i, j}^{ \pm}=\varphi_{j}^{ \pm}\left(f_{i}\right)$
(iii) $h_{i, j}^{ \pm}=\varphi_{j}^{ \pm}\left(h_{i}\right)$
(iv) $k_{j}^{ \pm}=\varphi_{j}^{ \pm}\left(h_{\theta}\right)($ see $(2.2))$. 
Now (2.15)(iii) together with (R6) implies that

$$
\begin{gathered}
{\left[\varphi_{j}^{-}(h), \varphi_{j}^{+}\left(h^{\prime}\right), x\right]=\alpha(h) \alpha\left(h^{\prime}\right) x, \quad x \in \mathcal{G}_{\alpha}, \alpha \in \Phi,} \\
{\left[\varphi_{j}^{-}(h), \varphi_{j}^{+}\left(h^{\prime}\right), y\right]=\alpha(h) \alpha\left(h^{\prime}\right) y, \quad y \in\left(\mathcal{V}^{r}\right)_{\alpha}, \alpha \in \Phi_{s h},} \\
1 \leq j \leq \nu, h, h^{\prime} \in H, 1 \leq r \leq m-1
\end{gathered}
$$

To figure out some more relations holding in $\mathcal{L}$, we need to introduce some new notation as follows:

$$
\begin{aligned}
& \mathfrak{h}_{\alpha, h^{\prime}}^{h,}:=\operatorname{span}_{\mathbb{C}}\left\{\alpha(h) \varphi_{j}^{ \pm}\left(h^{\prime}\right)-\alpha\left(h^{\prime}\right) \varphi_{j}^{ \pm}(h) \mid 1 \leq j \leq \nu\right\}, \alpha \in \Phi, h, h^{\prime} \in H . \\
& \mathfrak{h}_{\alpha}^{h, h^{\prime}}:=\operatorname{span}_{\mathbb{C}}\left\{\alpha(h) \varphi_{\sigma}\left(h^{\prime}\right)-\alpha\left(h^{\prime}\right) \varphi_{\sigma}(h) \mid \sigma \in \mathbb{Z}^{\nu}\right\},
\end{aligned}
$$

Since for $\alpha \in \Phi, x \in \mathcal{G}_{\alpha}$ and $h, h^{\prime} \in H$, we have $\left[\alpha(h) h^{\prime}-\alpha\left(h^{\prime}\right) h, x\right]=0,(2.10)$ implies that

$$
\left[\mathcal{G}_{\alpha}, \mathfrak{h}_{\alpha}^{h, h^{\prime}}\right]=0=\left[\mathcal{G}_{\alpha}, \mathfrak{h}_{\alpha, \nu}^{h, h^{\prime}}\right] ; \quad \alpha \in \Phi, \quad h, h^{\prime} \in H
$$

In particular for $1 \leq i \leq \ell$, we have
(i) $\left[\mathbb{C} e_{2}+\mathbb{C} f_{2}, \mathfrak{h}_{\varepsilon_{2}-\varepsilon_{3}}^{h_{1}, h_{\theta}}\right]=0$,
(ii) $\left[\mathbb{C} e_{\varepsilon_{1} \pm \varepsilon_{\ell}}+\mathbb{C} f_{\varepsilon_{1} \pm \varepsilon_{\ell}}, \mathfrak{h}_{\varepsilon_{1} \pm \varepsilon_{\ell}}^{h_{\ell}, h_{\theta}}\right]=0$,
(iii) $\left[\mathbb{C} e_{i}+\mathbb{C} f_{i}, \mathfrak{h}_{\alpha_{i}}^{H_{i}, h_{i+1}}\right]=0, i \neq \ell$,
(iv) $\left[\mathbb{C} e_{i}+\mathbb{C} f_{i}, \mathfrak{h}_{\alpha_{i}}^{H_{i}, h_{i-1}}\right]=0, i \neq 1$,
(v) $\left[e_{\varepsilon_{1}+\varepsilon_{2}}, \mathfrak{h}_{\varepsilon_{1}+\varepsilon_{2}}^{H_{1}, h_{\theta}}+\mathfrak{h}_{\varepsilon_{1}+\varepsilon_{2}}^{H_{2}, h_{\theta}}\right]=0$,
(vi) $\left[\mathbb{C} e_{i+1}+\mathbb{C} f_{i+1}, \mathfrak{h}_{\alpha_{i+1}}^{h_{i}, H_{i+1}}\right]=0, i \neq \ell$,
(vii) $\left[\mathbb{C}\left[e_{i+1}, e_{i}\right]+\mathbb{C}\left[f_{i+1}, f_{i}\right], \mathfrak{h}_{\alpha_{i}+\alpha_{i+1}}^{H_{i}, h_{i+1}}\right]=0, i \neq \ell$,
(viii) $\left[\mathbb{C}\left[e_{i-1}, e_{i}\right]+\mathbb{C}\left[f_{i-1}, f_{i}\right], \mathfrak{h}_{\alpha_{i-1}+\alpha_{i}}^{H_{i-1}, h_{i-1}}\right]=0, i \neq 1$,
$(i x)\left[\mathbb{C} e_{i}+\mathbb{C} f_{i}, \mathfrak{h}_{\alpha_{i}}^{H_{i}, h_{i}}\right]=0$.

Also considering (2.14), it follows using (2.15)( $i i i)$ and (R5)(ii) that

$$
\left[\mathfrak{h}_{\alpha, \nu}^{h, h^{\prime}},\left(\mathcal{V}^{r}\right)_{\alpha}\right]=0 ; \quad \alpha \in \Phi_{s h}, 1 \leq r \leq m-1, h, h^{\prime} \in H,
$$

in particular, contemplating (2.3), we have
(i) $\left[\mathfrak{h}_{\varepsilon_{1}, \nu}^{h_{1}, h_{\theta}}, v^{r}\right]=0$,
(ii) $\left[\mathfrak{h}_{\varepsilon_{\ell}, \nu}^{h_{\ell}, H_{1}+H_{\ell}},\left[e_{\ell}, f\right], v^{r}\right]=0$,
(iii) $\left[\mathfrak{h}_{\varepsilon_{1}, \nu}^{h_{\theta}, H_{1}}, v^{r}\right]=0$,
(v) $\left[\mathfrak{h}_{-\varepsilon_{2}, \nu}^{h_{\theta}, h_{1}},\left[f_{2}, f_{3}, \ldots, f_{\ell}, f\right], v^{r}\right]=0$,
(iv) $\left[\mathfrak{h}_{-\varepsilon_{\ell}, \nu}^{h_{\ell}, H_{1}-H_{\ell}},\left[f_{\ell}, f\right], v^{r}\right]=0$,

Next let $h \in H$ and $\alpha \in \Phi$ such that $\alpha(h)=0$. We know that there exists $h^{\prime} \in H$ such that $\alpha\left(h^{\prime}\right) \neq 0$. One can see that $\mathfrak{h}_{\alpha, \nu}^{h, h^{\prime}}=\sum_{j=1}^{\nu} \mathbb{C} \varphi_{j}^{ \pm}(h)$ and 
$\mathfrak{h}_{\alpha}^{h, h^{\prime}}=\operatorname{span}_{\mathbb{C}}\left\{\varphi_{\sigma}(h) \mid \sigma \in \mathbb{Z}^{\nu}\right\}$. Using these together with (2.19) and (2.17), we have the following:

If $h \in H, \alpha \in \Phi_{s h}$ and $\beta \in \Phi$ such that $\alpha(h)=0=\beta(h)$, then for $1 \leq j \leq \nu$ and $1 \leq r \leq m-1$, we have $\left[\varphi_{j}^{ \pm}(h),\left(\mathcal{V}^{r}\right)_{\alpha}\right]=0$ and for $\sigma \in \mathbb{Z}^{\nu}$, we have $\left[\varphi_{\sigma}(h), \mathcal{G}_{\beta}\right]=0$.

In particular for $2 \leq i \leq \ell, 1 \leq j \leq \nu, H_{i}=\sum_{t=i}^{\ell-1} h_{t}+\frac{1}{2} h_{\ell}$ and $f=\left[f_{\ell}, \ldots, f_{1}\right] \in$ $\mathcal{G}_{-\varepsilon_{1}}$, we have
(i) $\left[\varphi_{j}^{ \pm}\left(H_{i}\right), v^{r}\right]=0$,
(ii) $\left[h_{i, j}^{ \pm}, v^{r}\right]=0$,
(iii) $\left[\varphi_{j}^{ \pm}\left(H_{1}\right), v_{i}^{r}\right]=0$
(iv) $\left[k_{j}^{ \pm},\left[f_{\ell}, f\right], v^{r}\right]=0$,
(v) $\left[\varphi_{j}^{ \pm}\left(H_{1}\right), v_{2 \ell+1}^{r}\right]=0$,
(vi) $\left[k_{j}^{ \pm},\left[e_{\ell}, f\right], v^{r}\right]=0$,
(vii) $\left[h_{1, j}^{ \pm},\left[f_{i-1}, \ldots, f_{1}\right], v^{r}\right]=0, i \neq 2,(v i i i)\left[h_{i, j}^{ \pm}, f_{1}, v^{r}\right]=0, i \neq 2$.

One knows from $(2.20)($ iii $)$ and $(2.22)(i)$ that

$$
\begin{aligned}
{\left[k_{j}^{ \pm}, v^{r}\right] } & =\left[\varphi_{j}^{ \pm}\left(H_{1}\right)-\varphi_{j}^{ \pm}\left(H_{i}\right), v^{r}\right] \text { and } \\
{\left[k_{j}^{ \pm}, v^{r}\right] } & =\left[\varphi_{j}^{ \pm}\left(H_{1}\right)+\varphi_{j}^{ \pm}\left(H_{i}\right), v^{r}\right] \\
\text { where } 1 & \leq j \leq \nu, 2 \leq i \leq \ell, 1 \leq r \leq m-1 .
\end{aligned}
$$

Also if $1 \leq i, t, s \leq \ell$ and $\sigma \in \mathbb{Z}^{\nu},(2.21)$ implies that

$$
\begin{aligned}
& \text { (i) }\left[\varphi_{\sigma}\left(H_{i}\right), \mathbb{C} f_{\varepsilon_{t}}+\mathbb{C} e_{\varepsilon_{t}}\right]=0, \quad i \neq t, \\
& \text { (ii) }\left[\varphi_{\sigma}\left(H_{i} \pm H_{t}\right), \mathbb{C} f_{\varepsilon_{i} \mp \varepsilon_{t}}+\mathbb{C} e_{\varepsilon_{i} \mp \varepsilon_{t}}\right]=0, \quad i<t, \\
& \text { (iii) }\left[\varphi_{\sigma}\left(H_{i}\right), \mathbb{C} f_{\varepsilon_{s} \pm \varepsilon_{t}}+\mathbb{C} e_{\varepsilon_{s} \pm \varepsilon_{t}}\right]=0, \quad s<t, s \neq i, i \neq t .
\end{aligned}
$$

To reduce the amount of computation, we introduce some new notations. Consider (2.14) and define

$$
\begin{gathered}
H_{i, j}^{ \pm}:=\varphi_{j}^{ \pm}\left(H_{i}\right) \text { where } H_{i}=\sum_{t=i}^{\ell-1} h_{t}+\frac{1}{2} h_{\ell} ; \\
1 \leq i \leq \ell, 1 \leq j \leq \nu .
\end{gathered}
$$

One can correspond to $\sigma \in \mathbb{Z}^{\nu}$ with norm-tuple $\left(a_{1}, \ldots, a_{n}\right)$ and $1 \leq i \leq \ell$, two $n$-tuples $\left(m_{1}^{i}, \ldots, m_{n}^{i}\right)$ and $\left(m_{i, 1}, \ldots, m_{i, n}\right)$ where for $1 \leq s \leq n, m_{s}^{i}$ and $m_{i, s}$ are defined as follows:

$$
m_{s}^{i}:=H_{i, j}^{ \pm} \text {and } m_{i, s}:=h_{i, j}^{ \pm} \text {if } a_{s}=k_{j}^{ \pm} \text {for some } 1 \leq j \leq \nu .
$$

Proposition 2.2. Let $\alpha \in \Phi$ and $\sigma \in \mathbb{Z}^{\nu}$ with norm-tuple $\left(a_{1}, \ldots, a_{n}\right)$. If $\left\{x_{1}, \ldots, x_{n}\right\} \subset H$ is such that for all $1 \leq i \leq n, \alpha\left(x_{i}\right) \neq 0$. Then $\varphi_{\sigma}\left(\mathcal{G}_{\alpha}\right)=$ $\left(\mathcal{G}_{\sigma}\right)_{\alpha}=\left[c_{1}, \ldots, c_{n}, \mathcal{G}_{\alpha}\right]$ where $c_{i}(1 \leq i \leq n)$ is defined to be $\varphi_{j}^{ \pm}\left(x_{i}\right)$ if $a_{i}=k_{j}^{ \pm}$ for some $1 \leq j \leq \nu$. 
Proof. It follows using Lemma 2.1 together with $(2.18)(v)$ and $(2.24)(i i i)$ that

$$
\begin{aligned}
& \text { (i) } e_{\sigma}=\left[m_{1}^{1}, \ldots, m_{n}^{1}, e_{\theta}\right],(i i) e_{\sigma}=\left[m_{1}^{2}, \ldots, m_{n}^{2}, e_{\theta}\right] \\
& (\text { iii }) e_{\sigma}=\left[m_{1}^{1}+m_{1}^{i}, \ldots, m_{n}^{1}+m_{n}^{i}, e_{\theta}\right] ; 3 \leq i \leq \ell
\end{aligned}
$$

Using (2.10) together with $(2.27)(i i)$ and $(2.24)(i),(i i)$, we have and

$$
\begin{aligned}
\varphi_{\sigma}\left(e_{\varepsilon_{2}}\right)=-\frac{1}{2}\left[f_{\varepsilon_{1}}, e_{\sigma}\right]=-\frac{1}{2}\left[f_{\varepsilon_{1}}, m_{1}^{2}, \ldots, m_{n}^{2}, e_{\theta}\right] & =-\frac{1}{2}\left[m_{1}^{2}, \ldots, m_{n}^{2}, f_{\varepsilon_{1}}, e_{\theta}\right] \\
& =\left[m_{1}^{2}, \ldots, m_{n}^{2}, e_{\varepsilon_{2}}\right]
\end{aligned}
$$

and

$$
\begin{aligned}
\varphi_{\sigma}\left(f_{\varepsilon_{1}}\right)=\frac{1}{4}\left[f_{\varepsilon_{2}}, f_{\varepsilon_{1}}, f_{\varepsilon_{1}}, e_{\sigma}\right] & =\frac{1}{4}\left[f_{\varepsilon_{2}}, f_{\varepsilon_{1}}, f_{\varepsilon_{1}}, m_{1}^{2}, \ldots, m_{n}^{2}, e_{\theta}\right] \\
& =\frac{1}{4}\left[f_{\varepsilon_{2}}, m_{1}^{2}, \ldots, m_{n}^{2}, f_{\varepsilon_{1}}, f_{\varepsilon_{1}}, e_{\theta}\right] \\
& =\frac{1}{4}\left[f_{\varepsilon_{2}},-m_{1}^{1}, \ldots,-m_{n}^{1}, f_{\varepsilon_{1}}, f_{\varepsilon_{1}}, e_{\theta}\right] \\
& =\frac{1}{4}\left[-m_{1}^{1}, \ldots,-m_{n}^{1}, f_{\varepsilon_{2}}, f_{\varepsilon_{1}}, f_{\varepsilon_{1}}, e_{\theta}\right] \\
& =\left[-m_{1}^{1}, \ldots,-m_{n}^{1}, f_{\varepsilon_{1}}\right] .
\end{aligned}
$$

Also for $2 \leq i \leq \ell,(2.10)$ together with $(2.27)(i)$ and $(2.24)(i),(i i)$ gives that

$$
\begin{aligned}
\varphi_{\sigma}\left(f_{\varepsilon_{i}}\right)=-\frac{1}{4}\left[f_{\varepsilon_{1}}, f_{\varepsilon_{i}}, f_{\varepsilon_{2}}, e_{\sigma}\right] & =-\frac{1}{4}\left[f_{\varepsilon_{1}}, f_{\varepsilon_{i}}, f_{\varepsilon_{2}}, m_{1}^{1}, \ldots, m_{n}^{1}, e_{\theta}\right] \\
& =-\frac{1}{4}\left[f_{\varepsilon_{1}}, m_{1}^{1}, \ldots, m_{n}^{1}, f_{\varepsilon_{i}}, f_{\varepsilon_{2}}, e_{\theta}\right] \\
& =-\frac{1}{4}\left[f_{\varepsilon_{1}},-m_{1}^{i}, \ldots,-m_{n}^{i}, f_{\varepsilon_{i}}, f_{\varepsilon_{2}}, e_{\theta}\right] \\
& =-\frac{1}{4}\left[-m_{1}^{i}, \ldots,-m_{n}^{i}, f_{\varepsilon_{1}}, f_{\varepsilon_{i}}, f_{\varepsilon_{2}}, e_{\theta}\right] \\
& =\left[-m_{1}^{i}, \ldots,-m_{n}^{i}, f_{\varepsilon_{i}}\right]
\end{aligned}
$$

and

$$
\begin{aligned}
\varphi_{\sigma}\left(e_{\varepsilon_{1}}\right)=\frac{1}{2}\left[f_{\varepsilon_{2}}, e_{\sigma}\right]=\frac{1}{2}\left[f_{\varepsilon_{2}}, m_{1}^{1}, \ldots, m_{n}^{1}, e_{\theta}\right] & =\frac{1}{2}\left[m_{1}^{1}, \ldots, m_{n}^{1}, f_{\varepsilon_{2}}, e_{\theta}\right] \\
& =\left[m_{1}^{1}, \ldots, m_{n}^{1}, e_{\varepsilon_{1}}\right]
\end{aligned}
$$

Similarly for $3 \leq i \leq \ell,(2.10)$ together with $(2.27)($ iii $)$ and $(2.24)(i i),(i)$ implies 
that

$$
\begin{aligned}
\varphi_{\sigma}\left(e_{\varepsilon_{i}}\right)=\frac{1}{2}\left[f_{\varepsilon_{2}}, f_{\varepsilon_{1}-\varepsilon_{i}}, e_{\sigma}\right] & =\frac{1}{2}\left[f_{\varepsilon_{2}}, f_{\varepsilon_{1}-\varepsilon_{i}}, m_{1}^{1}+m_{1}^{i}, \ldots, m_{n}^{1}+m_{n}^{i}, e_{\theta}\right] \\
& =\frac{1}{2}\left[m_{1}^{1}+m_{1}^{i}, \ldots, m_{n}^{1}+m_{n}^{i}, f_{\varepsilon_{2}}, f_{\varepsilon_{1}-\varepsilon_{i}}, e_{\theta}\right] \\
& =\left[m_{1}^{1}+m_{1}^{i}, \ldots, m_{n}^{1}+m_{n}^{i}, e_{\varepsilon_{i}}\right] \\
& =\left[m_{1}^{i}, \ldots, m_{n}^{i}, e_{\varepsilon_{i}}\right]
\end{aligned}
$$

Up to now, we have proved that

$\varphi_{\sigma}\left(e_{\varepsilon_{i}}\right)=\left[m_{1}^{i}, \ldots, m_{n}^{i}, e_{\varepsilon_{i}}\right]$ and $\varphi_{\sigma}\left(f_{\varepsilon_{i}}\right)=\left[-m_{1}^{i}, \ldots,-m_{n}^{i}, f_{\varepsilon_{i}}\right] ; 1 \leq i \leq \ell$.

Now let $1 \leq i \neq j \leq \ell$. Using (2.10), (2.28) and (2.24)( $i$ ), we have

$$
\begin{aligned}
\varphi_{\sigma}\left(\left[f_{\varepsilon_{j}}, e_{\varepsilon_{i}}\right]\right) & =\left[f_{\varepsilon_{j}}, \varphi_{\sigma}\left(e_{\varepsilon_{i}}\right)\right]=\left[m_{1}^{i}, \ldots, m_{n}^{i}, f_{\varepsilon_{j}}, e_{\varepsilon_{i}}\right], \\
\varphi_{\sigma}\left(\left[f_{\varepsilon_{j}}, f_{\varepsilon_{i}}\right]\right) & =\left[f_{\varepsilon_{j}}, \varphi_{\sigma}\left(f_{\varepsilon_{i}}\right)\right]=\left[-m_{1}^{i}, \ldots,-m_{n}^{i}, f_{\varepsilon_{j}}, f_{\varepsilon_{i}}\right], \\
\varphi_{\sigma}\left(\left[e_{\varepsilon_{j}}, e_{\varepsilon_{i}}\right]\right) & =\left[e_{\varepsilon_{j}}, \varphi_{\sigma}\left(e_{\varepsilon_{i}}\right)\right]=\left[m_{1}^{i}, \ldots, m_{n}^{i}, e_{\varepsilon_{j}}, e_{\varepsilon_{i}}\right], \\
\varphi_{\sigma}\left(\left[e_{\varepsilon_{j}}, f_{\varepsilon_{i}}\right]\right) & =\left[e_{\varepsilon_{j}}, \varphi_{\sigma}\left(f_{\varepsilon_{i}}\right)\right]=\left[-m_{1}^{i}, \ldots,-m_{n}^{i}, e_{\varepsilon_{j}}, f_{\varepsilon_{i}}\right] .
\end{aligned}
$$

Using this and (2.28), we get

$$
\begin{gathered}
\left(\mathcal{G}_{\sigma}\right)_{ \pm \varepsilon_{j} \pm \varepsilon_{i}}=\left[m_{1}^{i}, \ldots, m_{n}^{i}, \mathcal{G}_{ \pm \varepsilon_{j} \pm \varepsilon_{i}}\right] \text { and }\left(\mathcal{G}_{\sigma}\right)_{ \pm \varepsilon_{i}}=\left[m_{1}^{i}, \ldots, m_{n}^{i}, \mathcal{G}_{ \pm \varepsilon_{i}}\right] \\
1 \leq i \neq j \leq \ell
\end{gathered}
$$

which together with (2.17) and Lemma 2.1 completes the proof.

Corollary 2.1. $\left[\mathfrak{h}, \sum_{\alpha \in \Phi \times} \sum_{\sigma \in \mathbb{Z}^{\nu}}\left(\mathcal{G}_{\sigma}\right)_{\alpha}\right] \subseteq \sum_{\alpha \in \Phi} \times \sum_{\sigma \in \mathbb{Z}^{\nu}}\left(\mathcal{G}_{\sigma}\right)_{\alpha}$

Proof. Fix $1 \leq i \leq \ell$ and $1 \leq j \leq \nu$. Let $\alpha \in \Phi^{\times}$and $\sigma \in \mathbb{Z}^{\nu}$ with norm-tuple $\left(a_{1}, \ldots, a_{n}\right)$. Then by Proposition 2.2 there is $\left\{c_{r}^{\alpha} \mid 1 \leq r \leq n\right\} \subseteq \mathfrak{h}$ such that $\left(\mathcal{G}_{\sigma}\right)_{\alpha}=\left[c_{1}^{\alpha}, \ldots, c_{n}^{\alpha}, \mathcal{G}_{\alpha}\right]$. Consider $(2.14)$ and define $\eta_{j}^{ \pm}=\sigma+\sigma_{j}^{ \pm}$. If $\alpha\left(h_{i}\right) \neq 0$, then by Lemma 2.1, (2.16) and Proposition 2.2, we have $\left(\mathcal{G}_{\eta_{j}^{ \pm}}\right)_{\alpha}=$ $\left[h_{i, j}^{ \pm}, c_{1}^{\alpha}, \ldots, c_{n}^{\alpha}, \mathcal{G}_{\alpha}\right]$, but if $\alpha\left(h_{i}\right)=0$, Lemma 2.1 together with (2.21) implies that $\left[h_{i, j}^{ \pm},\left(\mathcal{G}_{\sigma}\right)_{\alpha}\right]=\left[c_{1}^{\alpha}, \ldots, c_{n}^{\alpha}, h_{i, j}^{ \pm}, \mathcal{G}_{\alpha}\right]=0$. This completes the proof.

Remark 2. Let $1 \leq i \leq \ell$ and $\sigma \in \mathbb{Z}^{\nu}$ with norm-tuple $\left(a_{1}, \ldots, a_{n}\right)$. Using (2.29) and (2.28), we have 
(i) $e_{i, \sigma}=\left[m_{1}^{i}, \ldots, m_{n}^{i}, e_{i}\right]=($ if $i \neq \ell)\left[-m_{1}^{i+1}, \ldots,-m_{n}^{i+1}, e_{i}\right]$,

(ii) $f_{i, \sigma}=\left[-m_{1}^{i}, \ldots,-m_{n}^{i}, f_{i}\right]=($ if $i \neq \ell)\left[m_{1}^{i+1}, \ldots, m_{n}^{i+1}, f_{i}\right]$.

It follows using the first equality stated in $(i)$ together with (2.13)(ii), the Jacobi identity, (2.17) and Lemma 2.1 that

(iii) $\alpha_{i}(h)\left[\varphi_{j}^{ \pm}\left(h^{\prime}\right), h_{i, \sigma}\right]=\alpha_{i}\left(h^{\prime}\right)\left[\varphi_{j}^{ \pm}(h), h_{i, \sigma}\right]$ where $1 \leq j \leq \nu, h, h^{\prime} \in H$ and $\left\{\alpha_{t} \mid 1 \leq t \leq \ell\right\}$ is the base of $\Phi$.

\section{$\S 2.2 . \quad \mathcal{G}$-submodules isomorphic to $\mathcal{V}$}

For $1 \leq r \leq m-1$ and $\sigma \in \mathbb{Z}^{\nu}$, define

$v_{\sigma}^{r}:=\left[a_{1}, \ldots, a_{t}, v^{r}\right]$ where $\left(a_{1}, \ldots, a_{t}\right)$ is the norm-tuple of $\sigma$ and set $\mathcal{V}_{\sigma}^{r}$ to be the $\mathcal{G}$-submodule of $\mathcal{L}$ generated by $v_{\sigma}^{r}$.

Note that by $(\mathrm{R} 6)(i i), v_{0}^{r}=v^{r}$ and so $\mathcal{V}_{0}^{r}=\mathcal{V}^{r}$ (see (2.3)).

Proposition 2.3. Let $1 \leq r \leq m-1$. Then for $\sigma \in \mathbb{Z}^{\nu}, \mathcal{V}_{\sigma}^{r}$ is an irreducible finite dimensional $\mathcal{G}$-module of highest weight $\varepsilon_{1}$.

Proof. Considering $\sigma \in \mathbb{Z}^{\nu}$ and contemplating (2.5), we have $\psi\left(v_{\sigma}^{r}\right)=$ $v_{1} \otimes t^{\sigma} w_{r} \neq 0$ and so $v_{\sigma}^{r} \neq 0$. Therefore by [H, Theorem 21.4], it is enough to show

$$
\begin{aligned}
{\left[e_{i}, v_{\sigma}^{r}\right]=0,\left[h_{i}, v_{\sigma}^{r}\right] } & =\varepsilon_{1}\left(h_{i}\right) v_{\sigma}^{r},\left[f_{t}, v_{\sigma}^{r}\right]=\left[f_{1}, f_{1}, v_{\sigma}^{r}\right]=0 \\
1 & \leq i \leq \ell, 2 \leq t \leq \ell .
\end{aligned}
$$

Let $\left(a_{1}, \ldots, a_{n}\right)$ be the norm-tuple of $\sigma$. Then by (R3)(ii) and (R2), we have

$$
\left[h_{i}, v_{\sigma}^{r}\right]=\left[a_{1}, \ldots, a_{n}, h_{i}, v^{r}\right]=\left[a_{1}, \ldots, a_{n}, \varepsilon_{1}\left(h_{i}\right) v^{r}\right]=\varepsilon_{1}\left(h_{i}\right) v_{\sigma}^{r} ; 1 \leq i \leq \ell .
$$

Also by (R4)(ii) and (R2), we have

$$
\begin{aligned}
& {\left[f_{i}, v_{\sigma}^{r}\right]=\left[f_{i}, a_{1}, \ldots, a_{n}, v^{r}\right]=\left[a_{1}, \ldots, a_{n}, f_{i}, v^{r}\right]=0,3 \leq i \leq \ell, \text { and }} \\
& {\left[f_{1}, f_{1}, v_{\sigma}^{r}\right]=\left[f_{1}, f_{1}, a_{1}, \ldots, a_{n}, v^{r}\right]=\left[a_{1}, \ldots, a_{n}, f_{1}, f_{1}, v^{r}\right]=0 .}
\end{aligned}
$$

Thus it remains to prove $\left[f_{2}, v_{\sigma}^{r}\right]=0=\left[e_{i}, v_{\sigma}^{r}\right]$ for $1 \leq i \leq \ell$. We show this, using induction on $|\sigma|$. Fix $1 \leq i \leq \ell$. Since $\mathcal{V}_{0}^{r}=\mathcal{V}^{r}$, (R2) implies that the equalities hold for $\sigma=0$. Next let $\sigma, \tau \in \mathbb{Z}^{\nu}, v_{\sigma}^{r}=\left[a_{1}, \ldots, a_{n}, v^{r}\right]$ for some $n \in \mathbb{N}$ and $v_{\tau}^{r}=\left[k_{j}^{ \pm}, v_{\sigma}^{r}\right]$ for some $1 \leq j \leq \nu$ such that $\left[f_{2}, v_{\sigma}^{r}\right]=0=\left[e_{i}, v_{\sigma}^{r}\right]$. We prove $\left[f_{2}, v_{\tau}^{r}\right]=0=\left[e_{i}, v_{\tau}^{r}\right]$. By Lemma 2.1, (2.20)(i) and (2.15)(iv), we have

$$
\begin{aligned}
v_{\tau}^{r}=\left[k_{j}^{ \pm}, a_{1}, \ldots, a_{n}, v^{r}\right] & =\frac{1}{2}\left[a_{1}, \ldots, a_{n}, 2 k_{j}^{ \pm}, v^{r}\right] \\
& =\frac{1}{2}\left[a_{1}, \ldots, a_{n}, k_{j}^{ \pm}+h_{1, j}^{ \pm}, v^{r}\right]=\frac{1}{2}\left[k_{j}^{ \pm}+h_{1, j}^{ \pm}, v_{\sigma}^{r}\right] .
\end{aligned}
$$


This together with $(2.18)(i),(2.15)(i v)$ and the induction hypothesis implies that

$$
\begin{aligned}
& {\left[f_{2}, v_{\tau}^{r}\right]=\frac{1}{2}\left[f_{2}, k_{j}^{ \pm}+h_{1, j}^{ \pm}, v_{\sigma}^{r}\right]=\frac{1}{2}\left[k_{j}^{ \pm}+h_{1, j}^{ \pm}, f_{2}, v_{\sigma}^{r}\right]=0,} \\
& {\left[e_{2}, v_{\tau}^{r}\right]=\frac{1}{2}\left[e_{2}, k_{j}^{ \pm}+h_{1, j}^{ \pm}, v_{\sigma}^{r}\right]=\frac{1}{2}\left[k_{j}^{ \pm}+h_{1, j}^{ \pm}, e_{2}, v_{\sigma}^{r}\right]=0 .}
\end{aligned}
$$

Now let $i \neq 2$. Using (R4)(ii) together with the induction hypothesis, we have $\left[e_{i}, v_{\tau}^{r}\right]=\left[e_{i}, k_{j}^{ \pm}, v_{\sigma}^{r}\right]=\left[k_{j}^{ \pm}, e_{i}, v_{\sigma}^{r}\right]=0$. This completes the proof.

Now let $1 \leq r \leq m-1$ and $\sigma \in \mathbb{Z}^{\nu}$. Proposition 2.3 guarantees the existence of an isomorphism

$$
\psi_{\sigma}^{r}: \mathcal{V} \longrightarrow \mathcal{V}_{\sigma}^{r} \text { such that } v_{1} \mapsto v_{\sigma}^{r} \text {. }
$$

Set $\psi_{r}:=\psi_{0}^{r}$. Since $\psi_{r}\left(v_{1}\right)=v^{r},(2.3)$ and (1.7)(i) imply that

$$
v_{i}^{r}=\psi_{r}\left(v_{i}\right) ; \quad 1 \leq i \leq 2 \ell+1 .
$$

Also since $\mathcal{V}_{\sigma}^{r}$ is isomorphic to $\mathcal{V},(1.8)$ and $(1.7)(i)$ together with Lemmas 1.2 and 1.3 imply that $\mathcal{V}_{\sigma}^{r}$ admits a weight space decomposition relative to $H$ as follows:

$$
\begin{aligned}
& \mathcal{V}_{\sigma}^{r}=\left(\mathcal{V}_{\sigma}^{r}\right)_{0} \oplus \sum_{i=1}^{\ell}\left(\mathcal{V}_{\sigma}^{r}\right)_{ \pm \varepsilon_{i}} \text { with } \\
& \left(\mathcal{V}_{\sigma}^{r}\right)_{0}=\mathbb{C}\left(v_{\sigma}^{r}\right)_{2 \ell+1},\left(\mathcal{V}_{\sigma}^{r}\right)_{\varepsilon_{i}}=\mathbb{C}\left(v_{\sigma}^{r}\right)_{i},\left(\mathcal{V}_{\sigma}^{r}\right)_{-\varepsilon_{i}}=\mathbb{C}\left(v_{\sigma}^{r}\right)_{\ell+i} \text { where }
\end{aligned}
$$

for $1 \leq i \leq 2 \ell+1, \quad\left(v_{\sigma}^{r}\right)_{i}:=\psi_{\sigma}^{r}\left(v_{i}\right)$ satisfies the following

$$
\begin{aligned}
& \left(v_{\sigma}^{r}\right)_{1}=v_{\sigma}^{r},\left(v_{\sigma}^{r}\right)_{i}=\left[f_{i-1}, \ldots, f_{1}, v_{\sigma}^{r}\right]=\left[\left[f_{i-1}, \ldots, f_{1}\right], v_{\sigma}^{r}\right] ; 2 \leq i \leq \ell, \\
& \left(v_{\sigma}^{r}\right)_{2 \ell+1}=-\frac{1}{2}\left[f_{\ell}, \ldots, f_{1}, v_{\sigma}^{r}\right]=-\frac{1}{2}\left[f, v_{\sigma}^{r}\right] \text { where } f=\left[f_{\ell}, \ldots, f_{1}\right], \\
& \left(v_{\sigma}^{r}\right)_{\ell+i}=\frac{(-1)^{\ell-i}}{2}\left[f_{i}, \ldots, f_{\ell},\left(v_{\sigma}^{r}\right)_{2 \ell+1}\right] \\
& \quad=\frac{-(-1)^{\ell-i}}{4} \begin{cases}{\left[f_{1},\left[f_{2}, \ldots, f_{\ell}, f\right], v_{\sigma}^{r}\right]} & i=1, \\
{[} & \left.\left.f_{i}, \ldots, f_{\ell}, f\right], v_{\sigma}^{r}\right] \quad 2 \leq i \leq \ell .\end{cases}
\end{aligned}
$$

Next let $x, x_{1}, \ldots, x_{n} \in \mathcal{G}$. Since $\psi_{\sigma}^{r}$ is a $\mathcal{G}$-module isomorphism mapping $v_{1}$ to $v_{\sigma}^{r}$ (see $(2.32)$ ), we have $\psi_{\sigma}^{r}\left(x_{1} \cdots x_{n} \cdot v_{1}\right)=\left[x_{1}, \ldots, x_{n}, \psi_{\sigma}^{r}\left(v_{1}\right)\right]=$ $\left[x_{1}, \ldots, x_{n}, v_{\sigma}^{r}\right]$. Therefore $\left[x_{1}, \ldots, x_{n}, v_{\sigma}^{r}\right]=0$ if and only if $x_{1} \cdots x_{n} \cdot v_{1}=0$. Also if $1 \leq i, j \leq 2 \ell+1$ such that $x \cdot v_{i}=v_{j}$, then $\left[x,\left(v_{\sigma}^{r}\right)_{i}\right]=\left(v_{\sigma}^{r}\right)_{j}$. In particular for $f=\left[f_{\ell}, \ldots, f_{1}\right] \in \mathcal{G}_{-\varepsilon_{1}}$ and $1 \leq i \leq \ell$, considering (1.7), we have 
(i) $\left[f_{i},\left(v_{\sigma}^{r}\right)_{j}\right]= \begin{cases}\delta_{i, j}\left(v_{\sigma}^{r}\right)_{i+1} & i \neq \ell, 1 \leq j \leq \ell, \\ -2 \delta_{\ell, j}\left(v_{\sigma}^{r}\right)_{2 \ell+1} & i=\ell, 1 \leq j \leq \ell, \\ 2 \delta_{\ell, i}\left(v_{\sigma}^{r}\right)_{2 \ell} & j=2 \ell+1 \\ -\delta_{i+1, j-\ell}\left(v_{\sigma}^{r}\right)_{\ell+i} & \ell+1 \leq j \leq 2 \ell,\end{cases}$
(ii) $\left[f_{j}, f_{i}, f_{i-1}, \ldots, f_{2}, f_{1}, v_{\sigma}^{r}\right]=0, \quad i \neq \ell, 1 \leq j \neq i+1 \leq \ell$, $\left[f_{j}, f_{i}, f_{i+1}, \ldots, f_{\ell-1}, f_{\ell},\left(v_{\sigma}^{r}\right)_{2 \ell+1}\right]=0, \quad 1 \leq j \neq i-1 \leq \ell$,
(iii) $\left[f,\left[\mathbb{C} f_{\ell}+\mathbb{C} e_{\ell}, f\right], v_{\sigma}^{r}\right]=0, \quad(i v) \quad\left[\mathbb{C} e_{\varepsilon_{i}}+\mathbb{C} f_{\varepsilon_{i}}, v_{\sigma}^{r}\right]=0, i \neq 1$,
(v) $\left[e_{1},\left(v_{\sigma}^{r}\right)_{2 \ell+1}\right]=0$.

Now we are interested in finding expressions for the weight spaces of $\mathcal{V}_{\sigma}^{r}$ relative to the weight spaces of $\mathcal{V}^{r}$. Fix $2 \leq i \leq \ell$ and let $\left(a_{1}, \ldots, a_{n}\right)$ be the norm-tuple of $\sigma$. Using (2.34) together with (2.23), Lemma 2.1, (2.24)(ii), (2.3) and $(2.22)(i i i)$, we have

$$
\begin{aligned}
\left(\mathcal{V}_{\sigma}^{r}\right)_{\varepsilon_{i}} & =\mathbb{C}\left[\left[f_{i-1}, \ldots, f_{1}\right], a_{1}, \ldots, a_{n}, v^{r}\right] \\
& =\mathbb{C}\left[\left[f_{i-1}, \ldots, f_{1}\right], m_{1}^{1}+m_{1}^{i}, \ldots, m_{n}^{1}+m_{n}^{i}, v^{r}\right] \\
& =\mathbb{C}\left[m_{1}^{1}+m_{1}^{i}, \ldots, m_{n}^{1}+m_{n}^{i},\left[f_{i-1}, \ldots, f_{1}\right], v^{r}\right] \\
& =\left[m_{1}^{1}+m_{1}^{i}, \ldots, m_{n}^{1}+m_{n}^{i},\left(\mathcal{V}^{r}\right)_{\varepsilon_{i}}\right] \\
& =\left[m_{1}^{i}, \ldots, m_{n}^{i},\left(\mathcal{V}^{r}\right)_{\varepsilon_{i}}\right]
\end{aligned}
$$

and

$$
\begin{aligned}
\left(\mathcal{V}_{\sigma}^{r}\right)_{-\varepsilon_{i}} & =\mathbb{C}\left[\left[f_{i}, \ldots, f_{\ell}, f\right], a_{1}, \ldots, a_{n}, v^{r}\right] \\
& =\mathbb{C}\left[\left[f_{i}, \ldots, f_{\ell}, f\right], m_{1}^{1}-m_{1}^{i}, \ldots, m_{n}^{1}-m_{n}^{i}, v^{r}\right] \\
& =\mathbb{C}\left[m_{1}^{1}-m_{1}^{i}, \ldots, m_{n}^{1}-m_{n}^{i},\left[f_{i}, \ldots, f, f\right], v^{r}\right] \\
& =\left[m_{1}^{1}-m_{1}^{i}, \ldots, m_{n}^{1}-m_{n}^{i},\left(\mathcal{V}^{r}\right)_{-\varepsilon_{i}}\right] \\
& =\left[m_{1}^{i}, \ldots, m_{n}^{i},\left(\mathcal{V}^{r}\right)_{-\varepsilon_{i}}\right] .
\end{aligned}
$$

Also using (2.34) and the second equality of the above expression together 
with Lemma 2.1, $(2.20)(v),(\mathrm{R} 4)(i i)$ and (2.3), we have

$$
\begin{aligned}
\left(\mathcal{V}_{\sigma}^{r}\right)_{-\varepsilon_{1}}=\left[f_{1},\left(\mathcal{V}_{\sigma}^{r}\right)_{-\varepsilon_{2}}\right] & =\mathbb{C}\left[f_{1}, m_{1}^{1}-m_{1}^{2}, \ldots, m_{n}^{1}-m_{n}^{2},\left[f_{2}, \ldots, f_{\ell}, f\right], v^{r}\right] \\
& =\mathbb{C}\left[f_{1}, m_{1,1}, \ldots, m_{1, n},\left[f_{2}, \ldots, f_{\ell}, f\right], v^{r}\right] \\
& =\mathbb{C}(-1)^{n}\left[f_{1}, a_{1}, \ldots, a_{n},\left[f_{2}, \ldots, f_{\ell}, f\right], v^{r}\right] \\
& =\mathbb{C}\left[a_{1}, \ldots, a_{n}, f_{1},\left[f_{2}, \ldots, f_{\ell}, f\right], v^{r}\right] \\
& =\left[a_{1}, \ldots, a_{n},\left(\mathcal{V}^{r}\right)_{-\varepsilon_{1}}\right] .
\end{aligned}
$$

Summarizing our information, we have

$\left(\mathcal{V}_{\sigma}^{r}\right)_{ \pm \varepsilon_{1}}=\left[a_{1}, \ldots, a_{n},\left(\mathcal{V}^{r}\right)_{ \pm \varepsilon_{1}}\right], \quad\left(\mathcal{V}_{\sigma}^{r}\right)_{ \pm \varepsilon_{i}}=\left[m_{1}^{i}, \ldots, m_{n}^{i},\left(\mathcal{V}^{r}\right)_{ \pm \varepsilon_{i}}\right] ; 2 \leq i \leq \ell$.

More generally, we have the following proposition:

Proposition 2.4. Let $1 \leq r \leq m-1, \alpha \in \Phi_{\text {sh }}$ and $\sigma \in \mathbb{Z}^{\nu}$ with normtuple $\left(a_{1}, \ldots, a_{n}\right)$. If $\left\{x_{1}, \ldots, x_{n}\right\} \subset H$ is such that $\alpha\left(x_{i}\right) \neq 0,1 \leq i \leq n$. Then $\left(\mathcal{V}_{\sigma}^{r}\right)_{\alpha}=\left[c_{1}, \ldots, c_{n},\left(\mathcal{V}^{r}\right)_{\alpha}\right]$ where $c_{i}(1 \leq i \leq n)$ is defined to be $\varphi_{j}^{ \pm}\left(x_{i}\right)$ if $a_{i}=k_{j}^{ \pm}$for some $1 \leq j \leq \nu$.

Proof. Using (2.36) together with Lemma 2.1 and (2.19), we are done.

\section{§2.3. One-dimensional $\mathcal{G}$-submodules}

Up to now, we have introduced some irreducible $\mathcal{G}$-submodules of $\mathcal{L}$ whose highest weight is either a short root or a long root. Now we would like to introduce a trivial $\mathcal{G}$-submodule of $\mathcal{L}$. We recall that $f=\left[f_{\ell}, \ldots, f_{1}\right] \in \mathcal{G}_{-\varepsilon_{1}}$ and define

$$
\mathcal{D}:=\operatorname{span}_{\mathbb{C}}\left\{D_{\sigma, \tau}^{r, s}:=\left[\left[f, v_{\sigma}^{r}\right],\left[f, v_{\tau}^{s}\right]\right] \mid 1 \leq r, s \leq m-1, \sigma, \tau \in \mathbb{Z}^{\nu}\right\} .
$$

We claim that this subspace is a trivial $\mathcal{G}$-submodule. To prove, we need some lemmas.

Lemma 2.2. Let $1 \leq r \leq m-1$ and $\sigma \in \mathbb{Z}^{\nu}$ with norm-tuple $\left(a_{1}, \ldots, a_{n}\right)$. Then we have

(i) $\left[\left[f_{\ell}, f\right], v_{\sigma}^{r}\right]=\left[\left[f_{\ell}, f\right], a_{1}, \ldots, a_{n}, v^{r}\right]=\left(\frac{-1}{2}\right)^{n}\left[m_{\ell, 1}, \ldots, m_{\ell, n},\left[f_{\ell}, f\right], v^{r}\right]$.

(ii) $\left[\left[e_{\ell}, f\right], v_{\sigma}^{r}\right]=\left[\left[e_{\ell}, f\right], a_{1}, \ldots, a_{n}, v^{r}\right]=\left(\frac{1}{2}\right)^{n}\left[m_{\ell, 1}, \ldots, m_{\ell, n},\left[e_{\ell}, f\right], v^{r}\right]$. 
Proof. (i) Using the first part of (2.23) together with (2.24)(ii), (2.20)(iv) and Lemma 2.1, we have

$$
\begin{aligned}
{\left[\left[f_{\ell}, f\right], v_{\sigma}^{r}\right]=\left[\left[f_{\ell}, f\right], a_{1}, \ldots, a_{n}, v^{r}\right] } & =\left[\left[f_{\ell}, f\right], m_{1}^{1}-m_{1}^{\ell}, \ldots, m_{n}^{1}-m_{n}^{\ell}, v^{r}\right] \\
& =\left[m_{1}^{1}-m_{1}^{\ell}, \ldots, m_{n}^{1}-m_{n}^{\ell},\left[f_{\ell}, f\right], v^{r}\right] \\
& =\left(\frac{-1}{2}\right)^{n}\left[m_{\ell, 1}, \ldots, m_{\ell, n},\left[f_{\ell}, f\right], v^{r}\right] .
\end{aligned}
$$

(ii) Using the second part of (2.23) together with (2.24)(ii), (2.20)(ii) and Lemma 2.1, we have

$$
\begin{aligned}
{\left[\left[e_{\ell}, f\right], v_{\sigma}^{r}\right]=\left[\left[e_{\ell}, f\right], a_{1}, \ldots, a_{n}, v^{r}\right] } & =\left[\left[e_{\ell}, f\right], m_{1}^{1}+m_{1}^{\ell}, \ldots, m_{n}^{1}+m_{n}^{\ell}, v^{r}\right] \\
& =\left[m_{1}^{1}+m_{1}^{\ell}, \ldots, m_{n}^{1}+m_{n}^{\ell},\left[e_{\ell}, f\right], v^{r}\right] \\
& =\left(\frac{1}{2}\right)^{n}\left[m_{\ell, 1}, \ldots, m_{\ell, n},\left[e_{\ell}, f\right], v^{r}\right] .
\end{aligned}
$$

This completes the proof.

Lemma 2.3. For $\sigma, \tau \in \mathbb{Z}^{\nu}$ and $1 \leq r, s \leq m-1$, we have

(i) $\left[v_{\tau}^{s},\left[f_{\ell}, f\right], v_{\sigma}^{r}\right]=\left[v_{\sigma}^{r},\left[f_{\ell}, f\right], v_{\tau}^{s}\right]$,

(ii) $\left[v_{\tau}^{s},\left[e_{\ell}, f\right], v_{\sigma}^{r}\right]=\left[v_{\sigma}^{r},\left[e_{\ell}, f\right], v_{\tau}^{s}\right]$.

Proof. Let $v_{\sigma}^{r}=\left[a_{i_{1}}, \ldots, a_{i_{t}}, v^{r}\right]$ for some $t \in \mathbb{N}$. One knows that $\left[\left[f_{\ell}, f\right], v^{r}\right]$ $\in\left(\mathcal{V}^{r}\right)_{-\varepsilon_{\ell}}$ and $\left[\left[e_{\ell}, f\right], v^{r}\right] \in\left(\mathcal{V}^{r}\right)_{\varepsilon_{\ell}}$. So considering (2.3) and using (R7), Lemma 2.1 and (2.18)(ii), we have

$$
\begin{aligned}
& \text { (a) }\left[k_{j}^{ \pm}, v^{s},\left[f_{\ell}, f\right], v^{r}\right]=\frac{-1}{2}\left[h_{\ell, j}^{ \pm}, v^{s},\left[f_{\ell}, f\right], v^{r}\right], 1 \leq j \leq \nu . \\
& \text { (b) }\left[k_{j}^{ \pm}, v^{s},\left[e_{\ell}, f\right], v^{r}\right]=\frac{1}{2}\left[h_{\ell, j}^{ \pm}, v^{s},\left[e_{\ell}, f\right], v^{r}\right],
\end{aligned}
$$

(i) Let $\left(a_{j_{1}}, \ldots, a_{j_{n}}\right)$ be the norm-tuple of $\tau$. We first use induction on $|\tau|$ to prove

$\left[v_{\tau}^{s},\left[f_{\ell}, f\right], v_{\sigma}^{r}\right]=(-1 / 2)^{t+n}\left[m_{\ell, j_{1}}, \ldots, m_{\ell, j_{n}}, m_{\ell, i_{1}}, \ldots, m_{\ell, i_{t}}, v^{s},\left[f_{\ell}, f\right], v^{r}\right]$.

Let $|\tau|=0$, then the norm-tuple of $\tau$ is $\left(a_{j_{1}}, a_{j_{2}}\right)=\left(k_{1}^{-}, k_{1}^{+}\right)$and $v_{\tau}^{s}=$ $\left[a_{j_{1}}, a_{j_{2}}, v^{s}\right]=v^{s}$. We drew the attention of the reader to the point that by (R7) and (R6), we have

$$
\left(\frac{-1}{2}\right)^{2}\left[m_{\ell, j_{1}}, m_{\ell, j_{2}}, v^{s},\left[f_{\ell}, f\right], v^{r}\right]=\left[v^{s},\left[f_{\ell}, f\right], v^{r}\right] .
$$


This together with Lemma 2.2(i), (2.22)(ii) and Lemma 2.1 implies that

$$
\begin{aligned}
{\left[v_{\tau}^{s},\left[f_{\ell}, f\right], v_{\sigma}^{r}\right] } & =\left[v^{s},\left[f_{\ell}, f\right], v_{\sigma}^{r}\right] \\
& =(-1 / 2)^{t}\left[m_{\ell, i_{1}}, \ldots, m_{\ell, i_{t}}, v^{s},\left[f_{\ell}, f\right], v^{r}\right] \\
& =(-1 / 2)^{t+2}\left[m_{\ell, j_{1}}, m_{\ell, j_{2}}, m_{\ell, i_{1}}, \ldots, m_{\ell, i_{t}}, v^{s},\left[f_{\ell}, f\right], v^{r}\right] .
\end{aligned}
$$

So we have the first step of the induction. Next suppose $v_{\tau}^{s}=\left[a_{j_{1}}, \ldots, a_{j_{n}}, v^{s}\right]$ and $v_{\tau^{\prime}}^{s}=\left[k_{j}^{ \pm}, v_{\tau}^{s}\right]$ for some $1 \leq j \leq \nu$ such that (2.39) holds. Then by the Jacobi identity, Lemmas $2.2(i)$ and 2.1, $(2.22)(i v)$, the induction hypothesis and $(2.38)(a)$, we have

$$
\begin{aligned}
{\left[v_{\tau^{\prime}}^{s},\left[f_{\ell}, f\right], v_{\sigma}^{r}\right] } & =\left[\left[k_{j}^{ \pm},\left[f_{\ell}, f\right], v_{\sigma}^{r}\right], v_{\tau}^{s}\right]+\left[k_{j}^{ \pm}, v_{\tau}^{s},\left[f_{\ell}, f\right], v_{\sigma}^{r}\right] \\
& =\left(\frac{-1}{2}\right)^{t}\left[\left[m_{\ell, i_{1}}, \ldots, m_{\ell, i_{t}}, k_{j}^{ \pm},\left[f_{\ell}, f\right], v^{r}\right], v_{\tau}^{s}\right]+\left[k_{j}^{ \pm}, v_{\tau}^{s},\left[f_{\ell}, f\right], v_{\sigma}^{r}\right] \\
& =0+\left[k_{j}^{ \pm}, v_{\tau}^{s},\left[f_{\ell}, f\right], v_{\sigma}^{r}\right] \\
& =\left(\frac{-1}{2}\right)^{t+n}\left[m_{\ell, j_{1}}, \ldots, m_{\ell, j_{n}}, m_{\ell, i_{1}}, \ldots, m_{\ell, i_{t}}, k_{j}^{ \pm}, v^{s},\left[f_{\ell}, f\right], v^{r}\right] \\
& =\left(\frac{-1}{2}\right)^{t+n+1}\left[h_{\ell, j}^{ \pm}, m_{\ell, j_{1}}, \ldots, m_{\ell, j_{n}}, m_{\ell, i_{1}}, \ldots, m_{\ell, i_{t}}, v^{s},\left[f_{\ell}, f\right], v^{r}\right] .
\end{aligned}
$$

This completes the induction. Now considering Lemma 2.1, we are done as by (R7) we have $\left[v^{s},\left[f_{\ell}, f\right], v^{r}\right]=0$ for $r \neq s$.

(ii) Using Lemma 2.2(ii), (2.22)(vi) and (2.38)(b) in place of Lemma 2.2( $i)$, $(2.22)(i v)$ and $(2.38)(a)$ respectively in the proof of part $(i)$, one concludes that

$$
\left[v_{\tau}^{s},\left[e_{\ell}, f\right], v_{\sigma}^{r}\right]=(1 / 2)^{t+n}\left[m_{\ell, j_{1}}, \ldots, m_{\ell, j_{n}}, m_{\ell, i_{1}}, \ldots, m_{\ell, i_{t}}, v^{s},\left[e_{\ell}, f\right], v^{r}\right] .
$$

Now we are done, using (R7).

Proposition 2.5. $\quad \mathcal{D}$ is a trivial $\mathcal{G}$-submodule of $\mathcal{L}$.

Proof. Fix $\sigma, \tau \in \mathbb{Z}^{\nu}$ and $1 \leq r, s \leq m-1$. Since $\mathcal{G}$ is generated by $\left\{e_{i}, f_{i} \mid\right.$ $1 \leq i \leq \ell\}$, it is enough to show $\left[x, D_{\sigma, \tau}^{r, s}\right]=0$ for all $x \in\left\{e_{i}, f_{i} \mid 1 \leq i \leq \ell\right\}$. One knows that

$$
\left[f, f_{\ell-1}\right]=\left[f_{i}, f_{\ell}\right]=0,1 \leq i \leq \ell-2 \quad \text { and } \quad\left[f, e_{i}\right]=0,2 \leq i \leq \ell-1 .
$$

Therefore for $\gamma \in \mathbb{Z}^{\nu}$ and $1 \leq t \leq m-1$, the Jacobi identity together with $(2.35)(i),(2.34),(2.35)(v),(i i)$ and (2.31) implies that

$$
\begin{aligned}
& {\left[f_{\ell-1}, f, v_{\gamma}^{t}\right]=\left[f, f_{\ell-1}, v_{\gamma}^{t}\right]=0, \quad\left[e_{1}, f, \mathcal{V}_{\gamma}^{t}\right] \subset\left[e_{1},\left(\mathcal{V}_{\gamma}^{t}\right)_{0}\right]=0,} \\
& {\left[f_{i},\left[f, v_{\gamma}^{t}\right]\right]=\left[f_{i}, f_{\ell}, \ldots, f_{1}, v_{\gamma}^{t}\right]=\left[f_{\ell}, f_{i}, f_{\ell-1}, \ldots, f_{1}, v_{\gamma}^{t}\right]=0,1 \leq i \leq \ell-2,} \\
& {\left[e_{i}, f, v_{\gamma}^{t}\right]=\left[f, e_{i}, v_{\gamma}^{t}\right]=0,2 \leq i \leq \ell-1 .}
\end{aligned}
$$


This together with the Jacobi identity implies that

$$
\begin{gathered}
{\left[x, D_{\sigma, \tau}^{r, s}\right]=\left[x,\left[f, v_{\sigma}^{r}\right],\left[f, v_{\tau}^{s}\right]\right]=\left[\left[f, v_{\sigma}^{r}\right],\left[x,\left[f, v_{\tau}^{s}\right]\right]\right]-\left[\left[f, v_{\tau}^{s}\right],\left[x,\left[f, v_{\sigma}^{r}\right]\right]\right]=0} \\
x \in\left\{e_{i}, f_{i} \mid 1 \leq i \leq \ell-1\right\} .
\end{gathered}
$$

Now it remains to show $\left[x, D_{\sigma, \tau}^{r, s}\right]=0$ for $x \in\left\{e_{\ell}, f_{\ell}\right\}$. Let $x \in\left\{e_{\ell}, f_{\ell}\right\}$. Using the Jacobi identity together with $(2.31)$ and $(2.35)(i),($ iii $)$, we have

$$
\begin{aligned}
{\left[x, D_{\sigma, \tau}^{r, s}\right]=\left[x,\left[f, v_{\sigma}^{r}\right],\left[f, v_{\tau}^{s}\right]\right] } & =\left[\left[f, v_{\sigma}^{r}\right],\left[x,\left[f, v_{\tau}^{s}\right]\right]\right]-\left[\left[f, v_{\tau}^{s}\right],\left[x,\left[f, v_{\sigma}^{r}\right]\right]\right] \\
& =\left[\left[f, v_{\sigma}^{r}\right],\left[[x, f], v_{\tau}^{s}\right]\right]-\left[\left[f, v_{\tau}^{s}\right],\left[[x, f], v_{\sigma}^{r}\right]\right] \\
& =\left[f, v_{\sigma}^{r},[x, f], v_{\tau}^{s}\right]-\left[f, v_{\tau}^{s},[x, f], v_{\sigma}^{r}\right] .
\end{aligned}
$$

Now we are done, using Lemma 2.3.

\section{§2.4. The relations between introduced $\mathcal{G}$-submodules of $\mathcal{L}$}

As we promised we want to decompose $\mathcal{L}$ into a direct sum of irreducible $\mathcal{G}$-modules isomorphic to $\mathcal{G}$, irreducible $\mathcal{G}$-modules isomorphic to $\mathcal{V}$ and one dimensional $\mathcal{G}$-modules. For this, we need to know the relations between $\mathcal{D}$ and the irreducible $\mathcal{G}$-modules introduced in (2.7) and (2.30). We start with the following proposition:

Proposition 2.6. Let $1 \leq r \leq m-1, \sigma \in \mathbb{Z}^{\nu}$ and $\alpha \in \Phi$. Then $\left[v^{r},\left(\mathcal{G}_{\sigma}\right)_{\alpha}\right] \subseteq\left(\mathcal{V}_{\sigma}^{r}\right)_{\alpha+\varepsilon_{1}}$ where if $\alpha+\varepsilon_{1} \notin \Phi_{s h} \cup\{0\},\left(\mathcal{V}_{\sigma}^{r}\right)_{\alpha+\varepsilon_{1}}$ is defined to be zero.

Proof. Let $\left(a_{1}, \ldots, a_{n}\right)$ be the norm-tuple of $\sigma$. We first assume that $\alpha \in$ $\Phi \backslash\left\{ \pm \varepsilon_{1}, 0\right\}$, then there exists $h \in H$ such that $\varepsilon_{1}(h)=0$ and $\alpha(h) \neq 0$. Then Proposition 2.2 implies that $\left(\mathcal{G}_{\sigma}\right)_{\alpha}=\left[c_{1}, \ldots, c_{n}, \mathcal{G}_{\alpha}\right]$ where $c_{i}(1 \leq i \leq n)$ is defined to be $\varphi_{j}^{ \pm}(h)$ if $a_{i}=k_{j}^{ \pm}$for some $1 \leq j \leq \nu$. So by (2.21) and Propositions 2.3 and 2.4 , we have

$$
\left[v^{r},\left(\mathcal{G}_{\sigma}\right)_{\alpha}\right]=\left[c_{1}, \ldots, c_{n}, v^{r}, \mathcal{G}_{\alpha}\right] \subseteq\left[c_{1}, \ldots, c_{n},\left(\mathcal{V}^{r}\right)_{\alpha+\varepsilon_{1}}\right]=\left(\mathcal{V}_{\sigma}^{r}\right)_{\alpha+\varepsilon_{1}} ; \alpha \neq 0, \pm \varepsilon_{1} .
$$

Now let $\alpha=\varepsilon_{1}$. Using $(2.40)$, we have $\left[v^{r},\left(\mathcal{G}_{\sigma}\right)_{\varepsilon_{1}+\varepsilon_{2}}\right]=0$. This together with (2.10) and (2.35)(iv) implies that

$$
\left[v^{r},\left(\mathcal{G}_{\sigma}\right)_{\varepsilon_{1}}\right]=\mathbb{C}\left[v^{r}, f_{\varepsilon_{2}}, e_{\sigma}\right]=\mathbb{C}\left[f_{\varepsilon_{2}}, v^{r}, e_{\sigma}\right]=\left[f_{\varepsilon_{2}}, v^{r},\left(\mathcal{G}_{\sigma}\right)_{\varepsilon_{1}+\varepsilon_{2}}\right]=0 .
$$


Next let $\alpha=-\varepsilon_{1}$. One knows from $(2.40)$ that $\left[v^{r},\left(\mathcal{G}_{\sigma}\right)_{\varepsilon_{2}-\varepsilon_{1}}\right] \subseteq\left(\mathcal{V}_{\sigma}^{r}\right)_{\varepsilon_{2}}$ which together with (2.10), (2.35)(iv) and Proposition 2.3 implies that

$$
\left[v^{r},\left(\mathcal{G}_{\sigma}\right)_{-\varepsilon_{1}}\right]=\left[v^{r}, f_{\varepsilon_{2}},\left(\mathcal{G}_{\sigma}\right)_{\varepsilon_{2}-\varepsilon_{1}}\right]=\left[f_{\varepsilon_{2}}, v^{r},\left(\mathcal{G}_{\sigma}\right)_{\varepsilon_{2}-\varepsilon_{1}}\right] \subseteq\left[f_{\varepsilon_{2}},\left(\mathcal{V}_{\sigma}^{r}\right)_{\varepsilon_{2}}\right] \subseteq\left(\mathcal{V}_{\sigma}^{r}\right)_{0} .
$$

Finally let $\alpha=0$. For $2 \leq i \leq \ell$, (R2) implies that $\left[f_{i}, v^{r}\right]=0$ and (2.40) implies that $\left[v^{r},\left(\mathcal{G}_{\sigma}\right)_{\varepsilon_{1}-\varepsilon_{2}}\right]=\left[v^{r},\left(\mathcal{G}_{\sigma}\right)_{\alpha_{i}}\right]=0$ where $\left\{\alpha_{i} \mid 1 \leq i \leq \ell\right\}$ is the base of $\Phi$ introduced in $\S 1$. Therefore we have

$$
\left[v^{r},\left(\mathcal{G}_{\sigma}\right)_{\varepsilon_{1}-\varepsilon_{2}}\right]=0 \quad \text { and } \quad\left[v^{r}, f_{i},\left(\mathcal{G}_{\sigma}\right)_{\alpha_{i}}\right]=\left[f_{i}, v^{r},\left(\mathcal{G}_{\sigma}\right)_{\alpha_{i}}\right]=0,2 \leq i \leq \ell .
$$

Using (2.11) together with the above equalities, the Jacobi identity, Remark 2(i), (2.35)(i), (2.22)(iii) and Propositions 2.3 and 2.4, we have

$$
\begin{aligned}
{\left[v^{r},\left(\mathcal{G}_{\sigma}\right)_{0}\right] } & =\left[v^{r}, f_{1},\left(\mathcal{G}_{\sigma}\right)_{\varepsilon_{1}-\varepsilon_{2}}\right]+\sum_{i=2}^{\ell}\left[v^{r}, f_{i},\left(\mathcal{G}_{\sigma}\right)_{\alpha_{i}}\right] \\
& =\left[v^{r}, f_{1},\left(\mathcal{G}_{\sigma}\right)_{\varepsilon_{1}-\varepsilon_{2}}\right]+0 \\
& =\mathbb{C}\left[\left[v^{r}, f_{1}\right],\left[m_{1}^{1}, \ldots, m_{n}^{1}, e_{1}\right]\right]+\left[f_{1}, v^{r},\left(\mathcal{G}_{\sigma}\right)_{\varepsilon_{1}-\varepsilon_{2}}\right] \\
& =\mathbb{C}\left[m_{1}^{1}, \ldots, m_{n}^{1},\left[f_{1}, v^{r}\right], e_{1}\right]+0 \subseteq \mathbb{C}\left[m_{1}^{1}, \ldots, m_{n}^{1}, v^{r}\right]=\left(\mathcal{V}_{\sigma}^{r}\right)_{\varepsilon_{1}} .
\end{aligned}
$$

This completes the proof.

Now consider the set $\left\{\tau_{r}=\left(n_{1}^{r}, \ldots, n_{\nu}^{r}\right) \mid 1 \leq r \leq m-1\right\}$ of some representatives of nonzero cosets of $2 \mathbb{Z}^{\nu}$ in $\mathbb{Z}^{\nu}$ stated at the beginning of the section. We have the following proposition:

Proposition 2.7. Let $\sigma \in \mathbb{Z}^{\nu}$ and $1 \leq r, s \leq m-1$. Then $\left[v^{s},\left(\mathcal{V}_{\sigma}^{r}\right)_{\varepsilon_{1}}\right]=$ $\mathbb{C}\left[v^{s}, v_{\sigma}^{r}\right]=0$ and $\left[v^{s},\left(\mathcal{V}_{\sigma}^{r}\right)_{\alpha}\right] \subseteq\left(\mathcal{G}_{\sigma+\tau_{r}}\right)_{\varepsilon_{1}+\alpha}$ for $\alpha \in\{0\} \cup \Phi_{s h} \backslash\left\{ \pm \varepsilon_{1}\right\}$.

Proof. We first prove that for $\alpha \in\left\{0, \varepsilon_{t} \mid 2 \leq t \leq \ell\right\}$, we have $\left[v^{s},\left(\mathcal{V}_{\sigma}^{r}\right)_{\alpha}\right] \subseteq$ $\left(\mathcal{G}_{\sigma+\tau_{r}}\right)_{\varepsilon_{1}+\alpha}$. Let $2 \leq t \leq \ell$ and $\left(a_{1}, \ldots, a_{n}\right)$ be the norm-tuple of $\sigma$. Set

$$
a_{j}^{2, r}=\left\{\begin{array}{l}
\frac{1}{2} k_{j}^{+} \text {if } n_{j}^{r} \geq 0 \\
\frac{1}{2} k_{j}^{-} \text {if } n_{j}^{r}<0
\end{array} \text { and } a_{j}^{t, r}=\left\{\begin{array}{lll}
k_{j}^{+} \text {if } & n_{j}^{r} \geq 0 & 1 \leq j \leq \nu \\
k_{j}^{-} \text {if } & n_{j}^{r}<0 & t \neq 2 .
\end{array}\right.\right.
$$

Using (2.36) and (2.3), we have $\left(\mathcal{V}_{\sigma}^{r}\right)_{\varepsilon_{t}}=\left[m_{1}^{t}, \ldots, m_{n}^{t}, v_{t}^{r}\right]$. Therefore $(2.22)(i)$ together with (R7), (2.16) (if necessary) and Proposition 2.2 implies that

$$
\begin{aligned}
{\left[v^{s},\left(\mathcal{V}_{\sigma}^{r}\right)_{\varepsilon_{t}}\right] } & =\mathbb{C} \delta_{s, r}\left[m_{1}^{t}, \ldots, m_{n}^{t},\left(\operatorname{ad} a_{1}^{t, r}\right)^{\left|n_{1}^{r}\right|} \ldots\left(\operatorname{ad} a_{\nu}^{t, r}\right)^{\left|n_{\nu}^{r}\right|} e_{\varepsilon_{1}+\varepsilon_{t}}\right] \\
& \subseteq\left(\mathcal{G}_{\sigma+\tau_{r}}\right)_{\varepsilon_{1}+\varepsilon_{t}} .
\end{aligned}
$$


Next using (2.34) and (R2) together with (2.41) and Proposition 2.1, we have

$$
\begin{aligned}
{\left[v^{s},\left(\mathcal{V}_{\sigma}^{r}\right)_{0}\right]=\mathbb{C}\left[v^{s}, f_{\ell}, \ldots, f_{2}, f_{1}, v_{\sigma}^{r}\right] } & =\mathbb{C}\left[f_{\ell}, \ldots, f_{2}, v^{s}, f_{1}, v_{\sigma}^{r}\right] \\
& =\left[f_{\ell}, \ldots, f_{2}, v^{s},\left(\mathcal{V}_{\sigma}^{r}\right)_{\varepsilon_{2}}\right] \\
& \subseteq\left[f_{\ell}, \ldots, f_{2},\left(\mathcal{G}_{\sigma+\tau_{r}}\right)_{\varepsilon_{1}+\varepsilon_{2}}\right] \\
& \subseteq\left(\mathcal{G}_{\sigma+\tau_{r}}\right)_{\varepsilon_{1}} .
\end{aligned}
$$

Finally for $2 \leq t \leq \ell$, by (2.34), (R2), (2.42) and Proposition 2.1, we have

$$
\begin{aligned}
{\left[v^{s},\left(\mathcal{V}_{\sigma}^{r}\right)_{-\varepsilon_{t}}\right]=\mathbb{C}\left[v^{s}, f_{t}, \ldots, f_{\ell}, f, v_{\sigma}^{r}\right] } & =\mathbb{C}\left[f_{t}, \ldots, f_{\ell}, v^{s}, f, v_{\sigma}^{r}\right] \\
& =\left[f_{t}, \ldots, f_{\ell}, v^{s},\left(\mathcal{V}_{\sigma}^{r}\right)_{0}\right] \\
& \subseteq\left[f_{t}, \ldots, f_{\ell},\left(\mathcal{G}_{\sigma+\tau_{r}}\right)_{\varepsilon_{1}}\right] \\
& =\left(\mathcal{G}_{\sigma+\tau_{r}}\right)_{\varepsilon_{1}-\varepsilon_{t}} .
\end{aligned}
$$

Now considering (2.34) and using Proposition 2.3, we have $\left[e_{\varepsilon_{1}},\left(v_{\sigma}^{r}\right)_{2 \ell+1}\right]=$ $-v_{\sigma}^{r}$. This together with (2.31) implies that $\left[v^{s}, v_{\sigma}^{r}\right]=-\left[v^{s}, e_{\varepsilon_{1}},\left(v_{\sigma}^{r}\right)_{2 \ell+1}\right]=$ $-\left[e_{\varepsilon_{1}}, v^{s},\left(v_{\sigma}^{r}\right)_{2 \ell+1}\right]$. Therefore thanks to (2.42) and Proposition 2.1, we have

$$
\left[v^{s}, v_{\sigma}^{r}\right]=-\left[e_{\varepsilon_{1}}, v^{s},\left(v_{\sigma}^{r}\right)_{2 \ell+1}\right] \in-\left[e_{\varepsilon_{1}},\left(\mathcal{G}_{\sigma+\tau_{r}}\right)_{\varepsilon_{1}}\right]=0 .
$$

This completes the proof.

Lemma 2.4. Let $\sigma \in \mathbb{Z}^{\nu}$ and $1 \leq r, s \leq m-1$. Then for $1 \leq i \leq \ell-1$, we have

$$
\begin{aligned}
& {\left[\left[f_{i}, f_{i-1}, \ldots, f_{1}, v^{s}\right],\left[f_{i+1}, \ldots, f_{\ell}, f, v_{\sigma}^{r}\right]\right]} \\
& =\sum_{j=1}^{\ell-(i+1)}(-1)^{j+1}\left[f_{i+j}, \ldots, f_{1}, v^{s}, f_{i+j+1}, \ldots, f_{\ell}, f, v_{\sigma}^{r}\right] \\
& \quad+(-1)^{\ell-i+1}\left[f_{\ell}, \ldots, f_{1}, v^{s}, f, v_{\sigma}^{r}\right]+(-1)^{\ell-i}\left[\left[f, v^{s}\right],\left[f, v_{\sigma}^{r}\right]\right]
\end{aligned}
$$

where for $i=\ell-1, \sum_{j=1}^{\ell-(i+1)}(-1)^{j+1}\left[f_{i+j}, \ldots, f_{1}, v^{s}, f_{i+j+1}, \ldots, f_{\ell}, f, v_{\sigma}^{r}\right]$ is defined to be zero.

Proof. The proof will be carried out in steps:

(1) If $1 \leq j \leq \ell-1$, then $\left[\left[f_{j}, \ldots, f_{1}, v^{s}\right],\left[f, v_{\sigma}^{r}\right]\right]=\left[f_{j}, \ldots, f_{1}, v^{s}, f, v_{\sigma}^{r}\right]$ : We show this, using induction on $j$. Let $j=1$, then by the Jacobi identity, (2.34) and $(2.35)(i)$, we have

$$
\begin{aligned}
{\left[\left[f_{1}, v^{s}\right],\left[f, v_{\sigma}^{r}\right]\right] } & =\left[f_{1}, v^{s},\left[f, v_{\sigma}^{r}\right]\right]-\left[v^{s}, f_{1},\left[f, v_{\sigma}^{r}\right]\right] \\
& \left.=\left[f_{1}, v^{s},\left[f, v_{\sigma}^{r}\right]\right]+2\left[v^{s}, f_{1},\left(v_{\sigma}^{r}\right)_{2 \ell+1}\right]\right]=\left[f_{1}, v^{s}, f, v_{\sigma}^{r}\right]
\end{aligned}
$$


Now let the equality hold for $1 \leq j \leq \ell-2$. Using (2.34) and (2.35)(ii), we have $\left[f_{j+1},\left[f, v_{\sigma}^{r}\right]\right]=0$. This together with the Jacobi identity and the induction hypothesis implies that

$$
\begin{aligned}
{\left[\left[f_{j+1}, \ldots, f_{1}, v^{s}\right],\left[f, v_{\sigma}^{r}\right]\right] } & =\left[f_{j+1},\left[f_{j}, \ldots, f_{1}, v^{s}\right],\left[f, v_{\sigma}^{r}\right]\right] \\
& =\left[f_{j+1}, f_{j}, \ldots, f_{1}, v^{s}, f, v_{\sigma}^{r}\right] .
\end{aligned}
$$

This completes the induction.

(2) If $1 \leq j \leq i \leq \ell-2$, then $\left[\left[f_{j}, \ldots, f_{1}, v^{s}\right],\left[f_{i+2}, \ldots, f_{\ell}, f, v_{\sigma}^{r}\right]\right]=$ $\left[f_{j}, \ldots, f_{1}, v^{s}, f_{i+2}, \ldots, f_{\ell}, f, v_{\sigma}^{r}\right]:$ We use induction on $j$. Let $j=1$ and $1 \leq$ $i \leq \ell-2$. We first mention that by (2.34) and (2.35)(ii), $\left[f_{1}, f_{i+2}, \ldots, f_{\ell}, f, v_{\sigma}^{r}\right]$ $\in \mathbb{C}\left[f_{1}, f_{i+2}, \ldots, f_{\ell},\left(v_{\sigma}^{r}\right)_{2 \ell+1}\right]=0$ which together with the Jacobi identity implies that

$$
\begin{aligned}
{\left[\left[f_{1}, v^{s}\right],\left[f_{i+2}, \ldots, f_{\ell}, f, v_{\sigma}^{r}\right]\right] } & =\left[f_{1}, v^{s}, f_{i+2}, \ldots, f_{\ell}, f, v_{\sigma}^{r}\right]-0 \\
& =\left[f_{1}, v^{s}, f_{i+2}, \ldots, f_{\ell}, f, v_{\sigma}^{r}\right] .
\end{aligned}
$$

Next suppose that $\ell \geq 4$ and $1<j \leq \ell-3$ is such that the equality holds for $j \leq$ $i \leq \ell-2$. We show that the equality holds for $j+1 \leq i \leq \ell-2$. Let $j+1 \leq i \leq$ $\ell-2$. Since $j+1 \leq i,(2.34)$ and $(2.35)(i i)$ imply that $\left[f_{j+1}, f_{i+2}, \ldots, f_{\ell}, f, v_{\sigma}^{r}\right] \in$ $\mathbb{C}\left[f_{j+1}, f_{i+2}, \ldots, f_{\ell},\left(v_{\sigma}^{r}\right)_{2 \ell+1}\right]=0$. This together with the Jacobi identity and the induction hypothesis implies that

$$
\begin{aligned}
& {\left[\left[f_{j+1}, f_{j}, \ldots, f_{1}, v^{s}\right],\left[f_{i+2}, \ldots, f_{\ell}, f, v_{\sigma}^{r}\right]\right] } \\
= & {\left[f_{j+1},\left[f_{j}, \ldots, f_{1}, v^{s}\right],\left[f_{i+2}, \ldots, f_{\ell}, f, v_{\sigma}^{r}\right]\right]-0 } \\
= & {\left[f_{j+1}, f_{j}, \ldots, f_{1}, v^{s}, f_{i+2}, \ldots, f_{\ell}, f, v_{\sigma}^{r}\right] . }
\end{aligned}
$$

This completes the induction.

(3) It follows, using induction on $n=\ell-i$ by considering steps (1) and (2), that the equality stated in the lemma holds.

Proposition 2.8. Let $\sigma \in \mathbb{Z}^{\nu}$ and $1 \leq r, s \leq m-1$. Then $\left[v^{s},\left(\mathcal{V}_{\sigma}^{r}\right)_{-\varepsilon_{1}}\right] \subseteq$ $\mathcal{G}_{\sigma+\tau_{r}}+\mathcal{D}($ see $(2.37))$.

Proof. By (2.34), we get $\left(\mathcal{V}_{\sigma}^{r}\right)_{-\varepsilon_{1}}=\mathbb{C}\left[f_{1}, f_{2}, \ldots, f_{\ell}, f, v_{\sigma}^{r}\right]$. Now using the Jacobi identity and Lemma 2.4, we have

$$
\begin{aligned}
{\left[v^{s}, f_{1}, f_{2}, \ldots, f_{\ell}, f, v_{\sigma}^{r}\right] } & =\left[f_{1}, v^{s}, f_{2}, \ldots, f_{\ell}, f, v_{\sigma}^{r}\right]-\left[\left[f_{1}, v^{s}\right], f_{2}, \ldots, f_{\ell}, f, v_{\sigma}^{r}\right] \\
& =\sum_{i=1}^{\ell-1}(-1)^{i+1}\left[f_{i}, f_{i-1}, \ldots, f_{1}, v^{s}, f_{i+1}, \ldots, f_{\ell}, f, v_{\sigma}^{r}\right] \\
& +(-1)^{\ell}\left(-\left[f_{\ell}, \ldots, f_{1}, v^{s}, f, v_{\sigma}^{r}\right]+\left[\left[f, v^{s}\right],\left[f, v_{\sigma}^{r}\right]\right]\right)
\end{aligned}
$$


which together with Propositions 2.7 and 2.1 completes the proof as by (2.34), we have $\left[f, v_{\sigma}^{r}\right] \in\left(\mathcal{V}_{\sigma}^{r}\right)_{0}$ and $\left[f_{i+1}, \ldots, f_{\ell}, f, v_{\sigma}^{r}\right] \in\left(\mathcal{V}_{\sigma}^{r}\right)_{-\varepsilon_{i+1}}$ for $1 \leq i \leq \ell-1$.

Proposition 2.9. (i) Let $\sigma \in \mathbb{Z}^{\nu}$ and $1 \leq s \leq m-1$. Then $\left[\mathfrak{h},\left(\mathcal{V}_{\sigma}^{s}\right)_{0}\right]=0$. (ii) $\left[\mathfrak{h}, \sum_{s=1}^{m-1} \sum_{\sigma \in \mathbb{Z}^{\nu}} \mathcal{V}_{\sigma}^{s}\right] \subseteq \sum_{s=1}^{m-1} \sum_{\sigma \in \mathbb{Z}^{\nu}} \mathcal{V}_{\sigma}^{s}$.

Proof. (i) By (2.34), it is enough to show $\left[h_{i, j}^{ \pm}, f, v_{\sigma}^{s}\right]=0$ for all $1 \leq i \leq \ell$ and $1 \leq j \leq \nu$. Fix $1 \leq i \leq \ell, 1 \leq j \leq \nu$ and let $\left(a_{1}, \ldots, a_{n}\right)$ be the norm-tuple of $\sigma$. If $i \neq 1$, then by the Jacobi identity, (2.15)(iii), (2.21), Lemma 2.1 and $(2.22)(i i)$, we have

$$
\left[h_{i, j}^{ \pm}, f, v_{\sigma}^{s}\right]=\left[f, h_{i, j}^{ \pm}, v_{\sigma}^{s}\right]+\left[\left[h_{i, j}^{ \pm}, f\right], v_{\sigma}^{s}\right]=\left[f, a_{1}, \ldots, a_{n}, h_{i, j}^{ \pm}, v^{s}\right]+0=0 .
$$

Now let $i=1$. Then (2.34) together with (2.21), (2.36), (2.3), (2.22)(iii), Lemma 2.1 and (2.22)(vii) implies that

$$
\begin{aligned}
{\left[h_{1, j}^{ \pm}, f, v_{\sigma}^{s}\right] } & =\left[h_{1, j}^{ \pm}, f_{\ell},\left(v_{\sigma}^{s}\right)_{\ell}\right] \\
& =\left[f_{\ell}, h_{1, j}^{ \pm},\left(v_{\sigma}^{s}\right)_{\ell}\right] \\
& \in \mathbb{C}\left[f_{\ell}, h_{1, j}^{ \pm}, m_{1}^{1}+m_{1}^{\ell}, \ldots, m_{t}^{1}+m_{t}^{\ell}, v_{\ell}^{s}\right] \\
& =\mathbb{C}\left[f_{\ell}, m_{1}^{1}+m_{1}^{\ell}, \ldots, m_{t}^{1}+m_{t}^{\ell}, h_{1, j}^{ \pm},\left[f_{\ell-1}, \ldots, f_{1}\right], v^{s}\right]=0
\end{aligned}
$$

(ii) By part (i), it is enough to show $\left[\mathfrak{h}, \sum_{s=1}^{m-1} \sum_{\sigma \in \mathbb{Z}^{\nu}} \sum_{\beta \in \Phi_{s h}}\left(\mathcal{V}_{\sigma}^{s}\right)_{\beta}\right] \subseteq$ $\sum_{s=1}^{m-1} \sum_{\sigma \in \mathbb{Z}^{\nu}} \mathcal{V}_{\sigma}^{s}$. Fix $1 \leq i \leq \ell, 1 \leq j \leq \nu, 1 \leq r \leq m-1, \tau \in \mathbb{Z}^{\nu}$ and $\beta \in \Phi_{s h}$. Let $\left(a_{1}, \ldots, a_{n}\right)$ be the norm-tuple of $\tau$, then by Proposition 2.4, there exists a subset $\left\{c_{r}\right\}_{r=1}^{n} \subseteq \mathfrak{h}$ so that $\left(\mathcal{V}_{\tau}^{r}\right)_{\beta}=\left[c_{1}, \ldots, c_{n},\left(\mathcal{V}^{r}\right)_{\beta}\right]$. So Proposition 2.4 together with (possibly) (2.16) implies that $\left[h_{i, j}^{ \pm},\left(\mathcal{V}_{\tau}^{r}\right)_{\beta}\right]=\left[h_{i, j}^{ \pm}, c_{1}, \ldots, c_{t},\left(\mathcal{V}^{r}\right)_{\beta}\right] \subseteq$ $\sum_{s=1}^{m-1} \sum_{\sigma \in \mathbb{Z}^{\nu}} \mathcal{V}_{\sigma}^{s}$. This completes the proof.

Lemma 2.5. Consider (2.37), we have the following:

(i) $\left[v^{t}, \mathcal{D}\right] \subseteq \sum_{k=1}^{m-1} \sum_{\sigma \in \mathbb{Z}^{\nu}} \mathcal{V}_{\sigma}^{k}$ for $1 \leq t \leq m-1$.

(ii) $[\mathfrak{h}, \mathcal{D}]=0$.

Proof. (i) Let $1 \leq r, s \leq m-1$ and $\sigma, \tau \in \mathbb{Z}^{\nu}$. We show $\left[v^{t}, D_{\sigma, \tau}^{r, s}\right] \subseteq$ $\sum_{k=1}^{m-1} \sum_{\sigma \in \mathbb{Z}^{\nu}} \mathcal{V}_{\sigma}^{k}$. Note that by $(2.11)$ and Proposition 2.2, $\mathcal{G}_{\sigma+\tau_{r}}$ is generated by $\left\{e_{i}, f_{i} \mid 1 \leq i \leq \ell\right\} \cup \mathfrak{h}$. Now using the Jacobi identity, (2.34), Propositions 
$2.7,2.1,2.9(i i)$ and 2.3 , we have

$$
\begin{aligned}
{\left[v^{t}, D_{\sigma, \tau}^{r, s}\right]=} & -\left[\left[f, v_{\tau}^{s}\right], v^{t},\left[f, v_{\sigma}^{r}\right]\right]+\left[\left[f, v_{\sigma}^{r}\right], v^{t},\left[f, v_{\tau}^{s}\right]\right] \\
= & -\left[f, v_{\tau}^{s}, v^{t},\left[f, v_{\sigma}^{r}\right]\right]+\left[v_{\tau}^{s}, f, v^{t},\left[f, v_{\sigma}^{r}\right]\right] \\
& +\left[f, v_{\sigma}^{r}, v^{t},\left[f, v_{\tau}^{s}\right]\right]-\left[v_{\sigma}^{r}, f, v^{t},\left[f, v_{\tau}^{s}\right]\right] \\
\in & {\left[f, v_{\tau}^{s},\left(\mathcal{G}_{\sigma+\tau_{r}}\right)_{\varepsilon_{1}}\right]+\left[v_{\tau}^{s},\left(\mathcal{G}_{\sigma+\tau_{r}}\right)_{0}\right] } \\
& +\left[f, v_{\sigma}^{r},\left(\mathcal{G}_{\tau+\tau_{s}}\right)_{\varepsilon_{1}}\right]+\left[v_{\sigma}^{r},\left(\mathcal{G}_{\tau+\tau_{s}}\right)_{0}\right] \\
\subseteq & {\left[f, \sum_{k=1}^{m-1} \sum_{\sigma \in \mathbb{Z}^{\nu}} \mathcal{V}_{\sigma}^{k}\right]+\sum_{k=1}^{m-1} \sum_{\sigma \in \mathbb{Z}^{\nu}} \mathcal{V}_{\sigma}^{k} \subseteq \sum_{k=1}^{m-1} \sum_{\sigma \in \mathbb{Z}^{\nu}} \mathcal{V}_{\sigma}^{k} . }
\end{aligned}
$$

(ii) Let $1 \leq r, s \leq m-1$ and $\sigma, \tau \in \mathbb{Z}^{\nu}$. We need to prove $\left[\mathfrak{h}, D_{\sigma, \tau}^{r, s}\right]=0$. Using the Jacobi identity together with (2.34) and Proposition 2.9(i), we get

$$
\begin{aligned}
{\left[\mathfrak{h}, D_{\sigma, \tau}^{r, s}\right] } & =\left[\left[f, v_{\sigma}^{r}\right], \mathfrak{h},\left[f, v_{\tau}^{s}\right]\right]-\left[\left[f, v_{\tau}^{s}\right], \mathfrak{h},\left[f, v_{\sigma}^{r}\right]\right] \\
& \subseteq\left[\left[f, v_{\sigma}^{r}\right], \mathfrak{h},\left(\mathcal{V}_{\tau}^{s}\right)_{0}\right]-\left[\left[f, v_{\tau}^{s}\right], \mathfrak{h},\left(\mathcal{V}_{\sigma}^{r}\right)_{0}\right]=0 .
\end{aligned}
$$

This completes the proof.

\section{$\S 2.5$. Some central elements}

In this subsection, we are interested to know about the center of $\mathcal{L}$. Set

$$
\mathcal{Z}:=\operatorname{span}_{\mathbb{C}}\left\{\left[h_{i, \sigma}, h_{i, \tau}\right] \mid \sigma, \tau \in \mathbb{Z}^{\nu}, 1 \leq i \leq \ell\right\} .
$$

We claim that $\mathcal{Z}$ is contained in the center of $\mathcal{L}$. To prove, we need some lemmas:

Lemma 2.6. Consider (2.12) and (2.15). For $1 \leq i \leq \ell, 1 \leq j \leq \nu$ and $\sigma \in \mathbb{Z}^{\nu}$, we have

(i) $\left[e_{i+1, j}^{ \pm}, h_{i, \sigma}\right]=\left[e_{i+1, \sigma}, h_{i, j}^{ \pm}\right] \quad$ and $\left[f_{i+1, j}^{ \pm}, h_{i, \sigma}\right]=\left[f_{i+1, \sigma}, h_{i, j}^{ \pm}\right], i \neq \ell$,

(ii) $\left[e_{i-1, j}^{ \pm}, h_{i, \sigma}\right]=\left[e_{i-1, \sigma}, h_{i, j}^{ \pm}\right] \quad$ and $\left[f_{i-1, j}^{ \pm}, h_{i, \sigma}\right]=\left[f_{i-1, \sigma}, h_{i, j}^{ \pm}\right], i \neq 1$.

Proof. Let $\left(a_{1}, \ldots, a_{n}\right)$ be the norm-tuple of $\sigma$.

(i) Using Remark 2(i), (2.24)(iii),(i), Lemma 2.1, (2.18)(vii) and (2.21), we have

$$
\begin{aligned}
{\left[h_{i+1, j}^{ \pm}, e_{i+1}, e_{i, \sigma}\right] } & =\left[m_{1}^{i}, \ldots, m_{n}^{i}, h_{i+1, j}^{ \pm}, e_{i+1}, e_{i}\right] \\
& =\left\{\begin{array}{cc}
{\left[m_{1}^{i}, \ldots, m_{n}^{i}, H_{i, j}^{ \pm}, e_{i+1}, e_{i}\right] 1 \leq i \leq \ell-2} \\
0 & i=\ell-1 .
\end{array}\right.
\end{aligned}
$$


Also Remark 2(i) together with Lemma 2.1, (2.18)(iii) and (2.24)(iii),(i) implies that

$$
\begin{aligned}
{\left[e_{i+1}, h_{i+1, j}^{ \pm}, e_{i, \sigma}\right] } & =\left[e_{i+1}, m_{1}^{i}, \ldots, m_{n}^{i}, h_{i+1, j}^{ \pm}, e_{i}\right] \\
& = \begin{cases}-\left[e_{i+1}, m_{1}^{i}, \ldots, m_{n}^{i}, H_{i, j}^{ \pm}, e_{i}\right] & 1 \leq i \leq \ell-2 \\
-\left[e_{\ell}, m_{1}^{\ell-1}, \ldots, m_{n}^{\ell-1}, 2 H_{\ell-1, j}^{ \pm}, e_{\ell-1}\right] & i=\ell-1\end{cases} \\
& = \begin{cases}-\left[m_{1}^{i}, \ldots, m_{n}^{i}, H_{i, j}^{ \pm}, e_{i+1}, e_{i}\right] & 1 \leq i \leq \ell-2 \\
-\left[m_{1}^{\ell-1}, \ldots, m_{n}^{\ell-1}, 2 H_{\ell-1, j}^{ \pm}, e_{\ell}, e_{\ell-1}\right] & i=\ell-1 .\end{cases}
\end{aligned}
$$

Now the second equality in (2.13)(ii), the Jacobi identity and (2.13)(iii) together with the above equalities, Lemma 2.1, (2.24)(iii),(i),(ii), (2.18)(vi) and Remark 2(i) imply

$$
\begin{aligned}
{\left[e_{i+1, j}^{ \pm}, h_{i, \sigma}\right] } & =-\left[f_{i}, e_{i+1, j}^{ \pm}, e_{i, \sigma}\right]-\left[\left[e_{i+1, j}^{ \pm}, f_{i}\right], e_{i, \sigma}\right] \\
& =(1 / 2)\left[f_{i},\left[e_{i+1}, h_{i+1, j}^{ \pm}\right], e_{i, \sigma}\right]-0 \\
& =(1 / 2)\left[f_{i}, e_{i+1}, h_{i+1, j}^{ \pm}, e_{i, \sigma}\right]-(1 / 2)\left[f_{i}, h_{i+1, j}^{ \pm}, e_{i+1}, e_{i, \sigma}\right] \\
& =-\left[f_{i}, m_{1}^{i}, \ldots, m_{n}^{i}, H_{i, j}^{ \pm}, e_{i+1}, e_{i}\right] \\
& =-\left[f_{i}, m_{1}^{i}+m_{1}^{i+1}, \ldots, m_{n}^{i}+m_{n}^{i+1}, H_{i, j}^{ \pm}+H_{i+1, j}^{ \pm}, e_{i+1}, e_{i}\right] \\
& =-\left[m_{1}^{i}+m_{1}^{i+1}, \ldots, m_{n}^{i}+m_{n}^{i+1}, H_{i, j}^{ \pm}+H_{i+1, j}^{ \pm}, f_{i}, e_{i+1}, e_{i}\right] \\
& =\left[m_{1}^{i}+m_{1}^{i+1}, \ldots, m_{n}^{i}+m_{n}^{i+1}, H_{i, j}^{ \pm}+H_{i+1, j}^{ \pm}, e_{i+1}\right] \\
& =\left[m_{1}^{i+1}, \ldots, m_{n}^{i+1}, H_{i+1, j}^{ \pm}, e_{i+1}\right] \\
& =-\left[m_{1}^{i+1}, \ldots, m_{n}^{i+1}, h_{i, j}^{ \pm}, e_{i+1}\right] \\
& =-\left[h_{i, j}^{ \pm}, m_{1}^{i+1}, \ldots, m_{n}^{i+1}, e_{i+1}\right]=\left[e_{i+1, \sigma}, h_{i, j}^{ \pm}\right] .
\end{aligned}
$$

For the second statement use the same argument as above by replacing the first equality stated in (2.13)(ii) by the second one and Remark 2(ii) by Remark $2(i)$.

(ii) Using Remark 2(i), (2.24)(iii),(i),(ii), Lemma 2.1 and (2.18)(viii), we have

$$
\begin{aligned}
{\left[h_{i-1, j}^{ \pm}, e_{i-1}, e_{i, \sigma}\right] } & =\left[h_{i-1, j}^{ \pm}, e_{i-1}, m_{1}^{i}, \ldots, m_{n}^{i}, e_{i}\right] \\
& =\left[h_{i-1, j}^{ \pm}, e_{i-1}, m_{1}^{i}+m_{1}^{i-1}, \ldots, m_{n}^{i}+m_{n}^{i-1}, e_{i}\right] \\
& =\left[m_{1}^{i}+m_{1}^{i-1}, \ldots, m_{n}^{i}+m_{n}^{i-1}, h_{i-1, j}^{ \pm}, e_{i-1}, e_{i}\right] \\
& =\left[m_{1}^{i-1}, \ldots, m_{n}^{i-1}, H_{i-1, j}^{ \pm}, e_{i-1}, e_{i}\right] .
\end{aligned}
$$

Also Remark 2(i) together with Lemma 2.1, (2.18)(iv) and (2.24)(iii),(i),(ii) 
implies that

$$
\begin{aligned}
{\left[e_{i-1}, h_{i-1, j}^{ \pm}, e_{i, \sigma}\right] } & =\left[e_{i-1}, m_{1}^{i}, \ldots, m_{n}^{i}, h_{i-1, j}^{ \pm}, e_{i}\right] \\
& =-\left[e_{i-1}, m_{1}^{i}, \ldots, m_{n}^{i}, H_{i, j}^{ \pm}, e_{i}\right] \\
& =-\left[e_{i-1}, m_{1}^{i}+m_{1}^{i-1}, \ldots, m_{n}^{i}+m_{n}^{i-1}, H_{i, j}^{ \pm}+H_{i-1, j}^{ \pm}, e_{i}\right] \\
& =-\left[m_{1}^{i}+m_{1}^{i-1}, \ldots, m_{n}^{i}+m_{n}^{i-1}, H_{i, j}^{ \pm}+H_{i-1, j}^{ \pm}, e_{i-1}, e_{i}\right] \\
& =-\left[m_{1}^{i-1}, \ldots, m_{n}^{i-1}, H_{i-1, j}^{ \pm}, e_{i-1}, e_{i}\right] .
\end{aligned}
$$

Now the second equality in (2.13)(ii), the Jacobi identity and (2.13)(iii) together with the above equalities, (2.24)(iii),(i), (2.18)(iii) and Remark 2(i) imply

$$
\begin{aligned}
{\left[e_{i-1, j}^{ \pm}, h_{i, \sigma}\right] } & =-\left[f_{i}, e_{i-1, j}^{ \pm}, e_{i, \sigma}\right]-\left[\left[e_{i-1, j}^{ \pm}, f_{i}\right], e_{i, \sigma}\right] \\
& =(1 / 2)\left[f_{i},\left[e_{i-1}, h_{i-1, j}^{ \pm}\right], e_{i, \sigma}\right]-0 \\
& =(1 / 2)\left[f_{i}, e_{i-1}, h_{i-1, j}^{ \pm}, e_{i, \sigma}\right]-(1 / 2)\left[f_{i}, h_{i-1, j}^{ \pm}, e_{i-1}, e_{i, \sigma}\right] \\
& =-\left[f_{i}, m_{1}^{i-1}, \ldots, m_{n}^{i-1}, H_{i-1, j}^{ \pm}, e_{i-1}, e_{i}\right] \\
& =-\left[m_{1}^{i-1}, \ldots, m_{n}^{i-1}, H_{i-1, j}^{ \pm}, f_{i}, e_{i-1}, e_{i}\right] \\
& =\left\{\begin{array}{l}
{\left[m_{1}^{i-1}, \ldots, m_{n}^{i-1}, H_{i-1, j}^{ \pm}, e_{i-1}\right] i \neq \ell} \\
2\left[m_{1}^{\ell-1}, \ldots, m_{n}^{\ell-1}, H_{\ell-1, j}^{ \pm}, e_{\ell-1}\right] i=\ell \\
\end{array}=-\left[m_{1}^{i-1}, \ldots, m_{n}^{i-1}, h_{i, j}^{ \pm}, e_{i-1}\right]\right. \\
& =-\left[h_{i, j}^{ \pm}, m_{1}^{i-1}, \ldots, m_{n}^{i-1}, e_{i-1}\right]=\left[e_{i-1, \sigma}, h_{i, j}^{ \pm}\right] .
\end{aligned}
$$

For the last statement, use Remark $2(i i)$ and the first equality in (2.13)(ii) in place of Remark 2(ii) and the second equality in (2.13)(ii) respectively and repeat the same argument as above.

Lemma 2.7. For $\tau, \sigma \in \mathbb{Z}^{\nu}$ and $1 \leq i \leq \ell$, we have

$$
\left[e_{i, \sigma}, e_{i, \tau}\right]=0=\left[f_{i, \sigma}, f_{i, \tau}\right]
$$

Proof. We use induction on $|\sigma|$. If $|\sigma|=0$, then by (2.13)(i), we have $\left[e_{i, \sigma}, e_{i, \tau}\right]=0$ for all $\tau \in \mathbb{Z}^{\nu}$. Next assume $\left[e_{i, \sigma}, e_{i, \tau}\right]=0$ for all $\sigma, \tau \in \mathbb{Z}^{\nu}$ with $|\sigma|=t$. We show $\left[e_{i, \sigma^{\prime}}, e_{i, \tau}\right]=0$ for all $\sigma^{\prime}, \tau \in \mathbb{Z}^{\nu}$ with $\left|\sigma^{\prime}\right|=t+1$. Fix $\sigma^{\prime} \in \mathbb{Z}^{\nu}$ with $\left|\sigma^{\prime}\right|=t+1$. Then there exists $1 \leq j \leq \nu$ such that $\sigma^{\prime}=\sigma+\sigma_{j}^{ \pm}$ (see (2.14)) for some $\sigma \in \mathbb{Z}^{\nu}$ with $|\sigma|=t$. So by Lemma 2.1, $e_{\sigma^{\prime}}=\left[\frac{1}{2} k_{j}^{ \pm}, e_{\sigma}\right]$. Now let $\tau \in \mathbb{Z}^{\nu}$ with norm-tuple $\left(a_{1}, \ldots, a_{n}\right)$, then by Lemma 2.1 and (2.16), we have $\left[H_{i, j}^{ \pm}, H_{i, j}^{\mp}, e_{i}\right]=e_{i}$. So using Remark 2(i) and Lemma 2.1, we have 
$e_{i, \sigma^{\prime}}=\left[H_{i, j}^{ \pm}, e_{i, \sigma}\right]$ and $\left[H_{i, j}^{ \pm}, e_{i, \tau}\right]=e_{i, \tau^{\prime}}$ where $\tau^{\prime}=\tau+\sigma_{j}^{ \pm}$. Now the Jacobi identity together with the induction hypothesis implies that

$$
\left[e_{i, \sigma^{\prime}}, e_{i, \tau}\right]=\left[\left[H_{i, j}^{ \pm}, e_{i, \sigma}\right], e_{i, \tau}\right]=\left[H_{i, j}^{ \pm}, e_{i, \sigma}, e_{i, \tau}\right]-\left[e_{i, \sigma}, e_{i, \tau^{\prime}}\right]=0
$$

The second equality is similarly proved.

Lemma 2.8. For $\tau, \sigma \in \mathbb{Z}^{\nu}$ and $1 \leq i \leq \ell$, we have

(i) $\left[e_{i, \sigma}, h_{i, \tau}\right]=-\left[h_{i, \sigma}, e_{i, \tau}\right],(i i)\left[f_{i, \sigma}, h_{i, \tau}\right]=-\left[h_{i, \sigma}, f_{i, \tau}\right]$.

Proof. (i) Using (2.13)(ii), the Jacobi identity and Lemma 2.7, we get

$$
\left[e_{i, \sigma}, h_{i, \tau}\right]=-\left[e_{i, \sigma},\left[f_{i}, e_{i, \tau}\right]\right]=\left[f_{i}, e_{i, \tau}, e_{i, \sigma}\right]-\left[e_{i, \tau}, f_{i}, e_{i, \sigma}\right]=-\left[h_{i, \sigma}, e_{i, \tau}\right]
$$

The second statement is similarly proved.

Lemma 2.9. For $\tau \in \mathbb{Z}^{\nu}, 1 \leq j \leq \nu$ and $1 \leq i \leq \ell$, we have $\left[f_{i, \tau}, e_{i, j}^{ \pm}\right]=$ $-\left[H_{i, j}^{ \pm}, h_{i, \tau}\right]-h_{i, \tau^{ \pm}}$where $\tau^{ \pm}=\tau+\sigma_{j}^{ \pm}($see $(2.12))$.

Proof. Using Remark 2(i), the Jacobi identity, (2.13)(ii), Remark 2(ii) and possibly Lemma 2.1 and (2.16), we have

$$
\begin{aligned}
{\left[f_{i, \tau}, e_{i, j}^{ \pm}\right]=\left[H_{i, j}^{ \pm}, f_{i, \tau}, e_{i}\right]-\left[\left[H_{i, j}^{ \pm}, f_{i, \tau}\right], e_{i}\right] } & =-\left[H_{i, j}^{ \pm}, h_{i, \tau}\right]+\left[f_{i, \tau^{ \pm}}, e_{i}\right] \\
& =-\left[H_{i, j}^{ \pm}, h_{i, \tau}\right]-h_{i, \tau^{ \pm}}
\end{aligned}
$$

This completes the proof.

Proposition 2.10. $\mathcal{Z}=\operatorname{span}_{\mathbb{C}}\left\{\left[h_{i, \sigma}, h_{i, \tau}\right] \mid \sigma, \tau \in \mathbb{Z}^{\nu}, 1 \leq i \leq \ell\right\} \subseteq$ $Z(\mathcal{L})$. In particular $\mathcal{Z}$ is a trivial $\mathcal{G}$-submodule of $\mathcal{L}$.

Proof. We first prove that $\left[h_{i, j}^{ \pm}, h_{i, \sigma}\right] \in Z(\mathcal{L})$ for $1 \leq i \leq \ell, 1 \leq j \leq \nu$ and $\sigma \in \mathbb{Z}^{\nu}$. Since $\mathcal{L}$ is generated by $(2.1)$, it is enough to show that this generating set is contained in the centralizer of $\left[h_{i, j}^{ \pm}, h_{i, \sigma}\right]$ in $\mathcal{L}$. We start by proving that $\left[h_{r},\left[h_{i, j}^{ \pm}, h_{i, \sigma}\right]\right]=\left[f_{r},\left[h_{i, j}^{ \pm}, h_{i, \sigma}\right]\right]=\left[e_{r},\left[h_{i, j}^{ \pm}, h_{i, \sigma}\right]\right]=0$ for $1 \leq r \leq \ell$. Since $h_{r}=\left[e_{r}, f_{r}\right]$, it is enough to prove $\left[f_{r},\left[h_{i, j}^{ \pm}, h_{i, \sigma}\right]\right]=\left[e_{r},\left[h_{i, j}^{ \pm}, h_{i, \sigma}\right]\right]=0$. Also since the proofs of these equalities are similar, we just prove the first equality. By the Jacobi identity, (2.13)(iv),(iii) and (2.15)(i), we have

$$
\begin{aligned}
{\left[e_{r}, h_{i, j}^{ \pm}, h_{i, \sigma}\right] } & =\left[h_{i, j}^{ \pm}, e_{r}, h_{i, \sigma}\right]+\left[\left[e_{r}, h_{i, j}^{ \pm}\right], h_{i, \sigma}\right] \\
& =\left[h_{i, j}^{ \pm}, \frac{\alpha_{r}\left(h_{i}\right)}{2} e_{r}, h_{r, \sigma}\right]+\left[\frac{\alpha_{r}\left(h_{i}\right)}{2}\left[e_{r}, h_{r, j}^{ \pm}\right], h_{i, \sigma}\right] \\
& =-\alpha_{r}\left(h_{i}\right)\left(\left[h_{i, j}^{ \pm}, e_{r, \sigma}\right]+\left[e_{r, j}^{ \pm}, h_{i, \sigma}\right]\right) .
\end{aligned}
$$


Now we are done, using Lemmas 2.8 and 2.6 together with the fact that $\alpha_{r}\left(h_{i}\right)=0$ for $r \neq i, i-1, i+1$.

Next we want to prove that $\left[h_{r, a}^{ \pm}, h_{i, j}^{ \pm}, h_{i, \sigma}\right]=0$ for all $1 \leq a \leq \nu$ and $1 \leq r \leq \ell$. By Lemma 2.1, Remark 2(iii), (2.13)(ii) and the first part of the proof, we have

$$
\begin{aligned}
{\left[h_{r, a}^{ \pm}, h_{i, j}^{ \pm}, h_{i, \sigma}\right]=\left[h_{i, j}^{ \pm}, h_{r, a}^{ \pm}, h_{i, \sigma}\right] } & \in \mathbb{C}\left[h_{i, j}^{ \pm}, h_{i, a}^{ \pm}, h_{i, \sigma}\right] \\
& =\mathbb{C}\left[h_{i, a}^{ \pm}, h_{i, j}^{ \pm}, h_{i, \sigma}\right] \\
& =\mathbb{C}\left[e_{i},\left[f_{i, a}^{ \pm}, h_{i, j}^{ \pm}, h_{i, \sigma}\right]\right]-\mathbb{C}\left[f_{i, a}^{ \pm},\left[e_{i}, h_{i, j}^{ \pm}, h_{i, \sigma}\right]\right] \\
& =\mathbb{C}\left[e_{i},\left[f_{i, a}^{ \pm}, h_{i, j}^{ \pm}, h_{i, \sigma}\right]\right]-0 \\
& =\mathbb{C}\left[e_{i},\left[f_{i, a}^{ \pm}, H_{i, j}^{ \pm}, h_{i, \sigma}\right]\right],
\end{aligned}
$$

so it is enough to show $\left[f_{i, a}^{ \pm}, H_{i, j}^{ \pm}, h_{i, \sigma}\right]=0$. Set $\tau^{ \pm}:=\sigma+\sigma_{j}^{ \pm}$. Using Lemma 2.9, the Jacobi identity, Lemmas 2.8(ii) and 2.7, Remark 2(ii), Lemma 2.1, (2.18) $(i x)$ and (2.16) (if necessary), we have

$$
\begin{aligned}
{\left[f_{i, a}^{ \pm}, H_{i, j}^{ \pm}, h_{i, \sigma}\right] } & =-\left[f_{i, a}^{ \pm},\left[f_{i, \sigma}, e_{i, j}^{ \pm}\right]\right]-\left[f_{i, a}^{ \pm}, h_{i, \tau^{ \pm}}\right] \\
& =\left[\left[f_{i, a}^{ \pm}, e_{i, j}^{ \pm}\right], f_{i, \sigma}\right]-\left[e_{i, j}^{ \pm}, f_{i, \sigma}, f_{i, a}^{ \pm}\right]+\left[h_{i, a}^{ \pm}, f_{i, \tau^{ \pm}}\right] \\
& =\left[\left[f_{i, a}^{ \pm}, e_{i, j}^{ \pm}\right], f_{i, \sigma}\right]+0+2\left[H_{i, a}^{ \pm}, f_{i, \tau^{ \pm}}\right] \\
& =\left[\left[f_{i, a}^{ \pm}, e_{i, j}^{ \pm}\right], f_{i, \sigma}\right]-2\left[H_{i, a}^{ \pm}, H_{i, j}^{ \pm}, f_{i, \sigma}\right] .
\end{aligned}
$$

Now let $\left(a_{1}, \ldots, a_{n}\right)$ be the norm-tuple of $\sigma$. It follows using induction on $n$ together with (R9) that

$$
\left[\left[f_{i, a}^{ \pm}, e_{i, j}^{ \pm}\right],-m_{1}^{i}, \ldots,-m_{n}^{i}, f_{i}\right]=\left[-m_{1}^{i}, \ldots,-m_{n}^{i},\left[f_{i, a}^{ \pm}, e_{i, j}^{ \pm}\right], f_{i}\right]
$$

This together with Remark 2(ii), the Jacobi identity, (2.13)(i),(ii), Lemma 2.1, (2.15)(iii) and (2.18)(ix) implies that 


$$
\begin{aligned}
{\left[\left[f_{i, a}^{ \pm}, e_{i, j}^{ \pm}\right], f_{i, \sigma}\right] } & =\left[-m_{1}^{i}, \ldots,-m_{n}^{i},\left[f_{i, a}^{ \pm}, e_{i, j}^{ \pm}\right], f_{i}\right] \\
& =\left[-m_{1}^{i}, \ldots,-m_{n}^{i}, f_{i, a}^{ \pm}, e_{i, j}^{ \pm}, f_{i}\right]-\left[-m_{1}^{i}, \ldots,-m_{n}^{i}, e_{i, j}^{ \pm}, f_{i, a}^{ \pm}, f_{i}\right] \\
& =\left[-m_{1}^{i}, \ldots,-m_{n}^{i}, f_{i, a}^{ \pm}, h_{i, j}^{ \pm}\right] \\
& =\left[-m_{1}^{i}, \ldots,-m_{n}^{i},\left[-H_{i, a}^{ \pm}, f_{i}\right], h_{i, j}^{ \pm}\right] \\
& =\left[-m_{1}^{i}, \ldots,-m_{n}^{i}, h_{i, j}^{ \pm}, H_{i, a}^{ \pm}, f_{i}\right] \\
& =\left[-m_{1}^{i}, \ldots,-m_{n}^{i}, H_{i, a}^{ \pm}, h_{i, j}^{ \pm}, f_{i}\right] \\
& =2\left[-m_{1}^{i}, \ldots,-m_{n}^{i}, H_{i, a}^{ \pm}, H_{i, j}^{ \pm}, f_{i}\right] \\
& =2\left[H_{i, a}^{ \pm}, H_{i, j}^{ \pm}, f_{i, \sigma}\right]
\end{aligned}
$$

which together with (2.44) gives $\left[f_{i, a}^{ \pm}, H_{i, j}^{ \pm}, h_{i, \sigma}\right]=0$.

Finally, we prove that $\left[v^{r}, h_{i, j}^{ \pm}, h_{i, \sigma}\right]=0$ for $1 \leq r \leq m-1$. We first suppose $i=1$. Using $(2.13)(i i)$, Remark $2(i),(2.35)(i),(2.22)(v)$ and $(2.35)(v)$, we have

$$
\begin{aligned}
& {\left[v_{2 \ell+1}^{r}, h_{1, \sigma}\right]=-\left[v_{2 \ell+1}^{r}, f_{1}, m_{1}^{1}, \ldots, m_{n}^{1}, e_{1}\right]=0,} \\
& {\left[v_{2 \ell+1}^{r}, h_{1, j}^{ \pm}\right]=-\left[v_{2 \ell+1}^{r}, f_{1}, H_{1, j}^{ \pm}, e_{1}\right]=0 .}
\end{aligned}
$$

One knows that $\psi_{r}$ (see (2.32)) is a $\mathcal{G}$-module isomorphism mapping $v_{1}$ to $v^{r}$ and $v_{2 \ell+1}$ to $v_{2 \ell+1}^{r}$. Since $e_{\varepsilon_{1}} \cdot v_{2 \ell+1}=-v_{1}$, we have $v^{r}=-\left[e_{\varepsilon_{1}}, v_{2 \ell+1}^{r}\right]$. Now the Jacobi identity together with (2.45) and the first part of the proof implies that

$$
\left[v^{r}, h_{1, j}^{ \pm}, h_{1, \sigma}\right]=-\left[e_{\varepsilon_{1}}, v_{2 \ell+1}^{r}, h_{1, j}^{ \pm}, h_{1, \sigma}\right]+\left[v_{2 \ell+1}^{r}, e_{\varepsilon_{1}}, h_{1, j}^{ \pm}, h_{1, \sigma}\right]=0 .
$$

Next let $i \neq 1$. Using the Jacobi identity together with (2.13)(ii), Remark $2(i),(2.31)$ and $(2.22)(i)$, we have

$$
\begin{aligned}
{\left[v^{r}, h_{i, j}^{ \pm}, h_{i, \sigma}\right] } & =\left[\left[v^{r}, h_{i, j}^{ \pm}\right], h_{i, \sigma}\right]+\left[h_{i, j}^{ \pm}, v^{r}, h_{i, \sigma}\right] \\
& =-\left[\left[v^{r},\left[f_{i}, e_{i, j}^{ \pm}\right]\right], h_{i, \sigma}\right]-\left[h_{i, j}^{ \pm}, v^{r}, f_{i}, e_{i, \sigma}\right] \\
& =-\left[\left[v^{r},\left[f_{i}, H_{i, j}^{ \pm}, e_{i}\right]\right], h_{i, \sigma}\right]-\left[h_{i, j}^{ \pm}, v^{r}, f_{i}, m_{1}^{i}, \ldots, m_{n}^{i}, e_{i}\right] \\
& =-\left[0, h_{i, \sigma}\right]-0=0 .
\end{aligned}
$$

So up to now, we have proved $\left[h_{i, j}^{ \pm}, h_{i, \sigma}\right] \in Z(\mathcal{L})$. This together with Remark 2(iii) implies that

$$
\left[h_{t, j}^{ \pm}, h_{i, \sigma}\right] \in \mathbb{C}\left[h_{i, j}^{ \pm}, h_{i, \sigma}\right] \subseteq Z(\mathcal{L}) ; 1 \leq i, t \leq \ell, 1 \leq j \leq \nu, \sigma \in \mathbb{Z}^{\nu} .
$$

Next let $1 \leq i \leq \ell$ and $\sigma, \tau \in \mathbb{Z}^{\nu}$. Using the same argument as in Lemma 2.6 by replacing Lemma 2.1 by $(2.46)$, one gets 
$\left[e_{i+1, \tau}, h_{i, \sigma}\right]=\left[e_{i+1, \sigma}, h_{i, \tau}\right] \quad$ and $\left[f_{i+1, \tau}, h_{i, \sigma}\right]=\left[f_{i+1, \sigma}, h_{i, \tau}\right], i \neq \ell$, $\left[e_{i-1, \tau}, h_{i, \sigma}\right]=\left[e_{i-1, \sigma}, h_{i, \tau}\right]$ and $\left[f_{i-1, \tau}, h_{i, \sigma}\right]=\left[f_{i-1, \sigma}, h_{i, \tau}\right], i \neq 1$.

Now we are ready to prove $\left[h_{i, \sigma}, h_{i, \tau}\right] \in Z(\mathcal{L})$ for $\sigma, \tau \in \mathbb{Z}^{\nu}$ and $1 \leq i \leq \ell$. As before it is enough to show that the generating set (2.1) is contained in the centralizer of $\left[h_{i, \sigma}, h_{i, \tau}\right]$ in $\mathcal{L}$. Use the same argument as above by replacing (2.47) and (2.46) by Lemmas 2.6 and 2.1 respectively to conclude

$$
\begin{gathered}
{\left[h_{r},\left[h_{i, \sigma}, h_{i, \tau}\right]\right]=\left[e_{r},\left[h_{i, \sigma}, h_{i, \tau}\right]\right]=\left[f_{r},\left[h_{i, \sigma}, h_{i, \tau}\right]\right]=\left[v^{s},\left[h_{i, \sigma}, h_{i, \tau}\right]\right]=0} \\
1 \leq r \leq \ell, 1 \leq s \leq m-1 .
\end{gathered}
$$

So it remains to show $\left[h_{t, j}^{ \pm},\left[h_{i, \sigma}, h_{i, \tau}\right]\right]=0$ for $1 \leq t \leq \ell$ and $1 \leq j \leq \nu$. Using the Jacobi identity together with (2.46), we have

$$
\left[h_{t, j}^{ \pm},\left[h_{i, \sigma}, h_{i, \tau}\right]\right]=\left[h_{i, \sigma},\left[h_{t, j}^{ \pm}, h_{i, \tau}\right]\right]+\left[\left[h_{t, j}^{ \pm}, h_{i, \sigma}\right], h_{i, \tau}\right]=0
$$

This completes the proof.

\section{§2.6. The proof of the main theorem}

Using the information in the previous subsections, we can decompose $\mathcal{L}$ into irreducible $\mathcal{G}$-modules. In fact we have the following theorem:

Theorem 2.2. $\quad$ Considering (2.37) and (2.43), we have

$$
\mathcal{L}=\sum_{\sigma \in \mathbb{Z}^{\nu}} \mathcal{G}_{\sigma}+\sum_{r=1}^{m-1} \sum_{\sigma \in \mathbb{Z}^{\nu}} \mathcal{V}_{\sigma}^{r}+\mathcal{D}+\mathcal{Z}
$$

Proof. Since $\mathcal{L}$ is generated by (2.1), it is enough to show that the right hand side of the equality in the statement is an ideal of $\mathcal{L}$ consisting of the generators. Using $(2.15)(i i i)$, we have $h_{i, j}^{ \pm} \in \mathcal{G}_{\sigma_{j}^{ \pm}} \subseteq \sum_{\sigma \in \mathbb{Z}^{\nu}} \mathcal{G}_{\sigma}$ for $1 \leq i \leq \ell$ and $1 \leq j \leq \nu$. Also we know $\left\{e_{i}, f_{i}, h_{i} \mid 1 \leq i \leq \ell\right\} \subseteq \mathcal{G}$ and for $1 \leq s \leq m-1$, $v^{s} \in \mathcal{V}_{0}^{s} \subseteq \sum_{r=1}^{m-1} \sum_{\sigma \in \mathbb{Z}^{\nu}} \mathcal{V}_{\sigma}^{r}$. Therefore we need to show that the right hand side of the equality is preserved under (left) multiplication by the generators. 
Let $\sigma \in \mathbb{Z}^{\nu}, 1 \leq i \leq \ell$ and $1 \leq j \leq \nu$. Using (2.11) together with (2.46) and Corollary 2.1, we have

$$
\begin{aligned}
{\left[h_{i, j}^{ \pm}, \mathcal{G}_{\sigma}\right] } & =\left[h_{i, j}^{ \pm},\left(\mathcal{G}_{\sigma}\right)_{0}\right]+\left[h_{i, j}^{ \pm}, \sum_{\alpha \in \Phi^{\times}}\left(\mathcal{G}_{\sigma}\right)_{\alpha}\right] \\
& =\mathbb{C}\left[h_{i, j}^{ \pm}, \sum_{t=1}^{\ell} h_{t, \sigma}\right]+\left[h_{i, j}^{ \pm}, \sum_{\alpha \in \Phi^{\times}}\left(\mathcal{G}_{\sigma}\right)_{\alpha}\right] \\
& \subseteq \mathcal{Z}+\sum_{\tau \in \mathbb{Z}^{\nu}} \mathcal{G}_{\tau} .
\end{aligned}
$$

Using this together with Lemma 2.5 and Propositions 2.1, 2.3, 2.5, 2.10, 2.6, $2.7,2.8$ and 2.9 , we are done.

Proposition 2.11. $\quad \mathcal{L}$ is a $B_{\ell}$-graded Lie algebra.

Proof. We know that the finite dimensional simple Lie algebra $\mathcal{G}$ is a subalgebra of $\mathcal{L}$. Also Theorem 2.2 shows that $\mathcal{L}$ admits a weight space decomposition $\mathcal{L}=\bigoplus_{\alpha \in \Phi} \mathcal{L}_{\alpha}$ relative to $H$ as follows:

$$
\begin{aligned}
& \mathcal{L}_{0}=\sum_{\sigma \in \mathbb{Z}^{\nu}}\left(\mathcal{G}_{\sigma}\right)_{0}+\sum_{r=1}^{m-1} \sum_{\sigma \in \mathbb{Z}^{\nu}}\left(\mathcal{V}_{\sigma}^{r}\right)_{0}+\mathcal{D}+\mathcal{Z}(\text { see }(2.37) \text { and }(2.43)) \\
& \mathcal{L}_{ \pm \varepsilon_{i}}=\sum_{\sigma \in \mathbb{Z}^{\nu}}\left(\mathcal{G}_{\sigma}\right)_{ \pm \varepsilon_{i}}+\sum_{r=1}^{m-1} \sum_{\sigma \in \mathbb{Z}^{\nu}}\left(\mathcal{V}_{\sigma}^{r}\right)_{ \pm \varepsilon_{i}}, 1 \leq i \leq \ell \\
& \mathcal{L}_{ \pm\left(\varepsilon_{i} \pm \varepsilon_{j}\right)}=\sum_{\sigma \in \mathbb{Z}^{\nu}}\left(\mathcal{G}_{\sigma}\right)_{ \pm\left(\varepsilon_{i} \pm \varepsilon_{j}\right)}, 1 \leq i<j \leq \ell
\end{aligned}
$$

So it remains to prove $\mathcal{L}_{0}=\sum_{\alpha \in \Phi^{\times}}\left[\mathcal{L}_{\alpha}, \mathcal{L}_{-\alpha}\right]$. Fix $1 \leq i, t \leq \ell, 1 \leq r, s \leq m-1$ and $\sigma, \tau \in \mathbb{Z}^{\nu}$. Using (2.48) together with (2.11) and (2.34), we have

$$
\begin{gathered}
\left(\mathcal{G}_{\sigma}\right)_{0} \subseteq \sum_{t=1}^{\ell}\left[\mathcal{G}_{-\alpha_{t}},\left(\mathcal{G}_{\sigma}\right)_{\alpha_{t}}\right] \subseteq \sum_{t=1}^{\ell}\left[\mathcal{L}_{-\alpha_{t}}, \mathcal{L}_{\alpha_{t}}\right] \subseteq \sum_{\alpha \in \Phi^{\times}}\left[\mathcal{L}_{\alpha}, \mathcal{L}_{-\alpha}\right] \\
\left(\mathcal{V}_{\sigma}^{r}\right)_{0}=\mathbb{C}\left[f, v_{\sigma}^{r}\right]=\left[\mathcal{G}_{-\varepsilon_{1}},\left(\mathcal{V}_{\sigma}^{r}\right)_{\varepsilon_{1}}\right] \subseteq\left[\mathcal{L}_{-\varepsilon_{1}}, \mathcal{L}_{\varepsilon_{1}}\right] \subseteq \sum_{\alpha \in \Phi^{\times}}\left[\mathcal{L}_{\alpha}, \mathcal{L}_{-\alpha}\right] .
\end{gathered}
$$

Also since $f \in \mathcal{L}_{-\varepsilon_{1}}$ and $v_{\sigma}^{r}, v_{\tau}^{s} \in \mathcal{L}_{\varepsilon_{1}}$, we have $\left[f, v_{\tau}^{s}\right] \in \mathcal{L}_{0},\left[f, f, v_{\tau}^{s}\right] \in \mathcal{L}_{-\varepsilon_{1}}$ and $\left[v_{\sigma}^{r},\left[f, v_{\tau}^{s}\right]\right] \in \mathcal{L}_{\varepsilon_{1}}$. Therefore the Jacobi identity implies that

$$
D_{\sigma, \tau}^{r, s}=\left[f, v_{\sigma}^{r},\left[f, v_{\tau}^{s}\right]\right]-\left[v_{\sigma}^{r}, f,\left[f, v_{\tau}^{s}\right]\right] \in\left[\mathcal{L}_{\varepsilon_{1}}, \mathcal{L}_{-\varepsilon_{1}}\right] \subseteq \sum_{\alpha \in \Phi^{\times}}\left[\mathcal{L}_{\alpha}, \mathcal{L}_{-\alpha}\right] .
$$

Finally by (2.48), we have $e_{i} \in \mathcal{G}_{\alpha_{i}} \subseteq \mathcal{L}_{\alpha_{i}}, h_{i, \tau} \in\left(\mathcal{G}_{\tau}\right)_{0} \subseteq \mathcal{L}_{0}$ and $f_{i, \sigma} \in$ $\left(\mathcal{G}_{\sigma}\right)_{-\alpha_{i}} \subseteq \mathcal{L}_{-\alpha_{i}}$, so $\left[f_{i, \sigma}, h_{i, \tau}\right] \in \mathcal{L}_{-\alpha_{i}}$ and $\left[e_{i}, h_{i, \tau}\right] \in \mathcal{L}_{\alpha_{i}}$. Therefore the Jacobi 
identity together with (2.13)(ii) implies that

$$
\begin{aligned}
{\left[h_{i, \sigma}, h_{i, \tau}\right]=\left[\left[e_{i}, f_{i, \sigma}\right], h_{i, \tau}\right] } & =\left[e_{i}, f_{i, \sigma}, h_{i, \tau}\right]-\left[f_{i, \sigma}, e_{i}, h_{i, \tau}\right] \\
& \in\left[\mathcal{L}_{\alpha_{i}}, \mathcal{L}_{-\alpha_{i}}\right] \subseteq \sum_{\alpha \in \Phi^{\times}}\left[\mathcal{L}_{\alpha}, \mathcal{L}_{-\alpha}\right] .
\end{aligned}
$$

This completes the proof.

Lemma 2.10. The center of $\mathcal{L}$ is contained in $\mathcal{Z}+\mathcal{D}$.

Proof. Let $x \in \sum_{\sigma \in \mathbb{Z}^{\nu}} \mathcal{G}_{\sigma}, y \in \sum_{r=1}^{m-1} \sum_{\sigma \in \mathbb{Z}^{\nu}} \mathcal{V}_{\sigma}^{r}, d \in \mathcal{D}$ and $z \in \mathcal{Z}$ such that $x+y+d+z \in Z(\mathcal{L})$. Then for $a \in \mathcal{G}$, Propositions 2.5 and 2.10 together with Propositions 2.1 and 2.3 imply that

$$
0=[a, x+y+d+z]=[a, x]+[a, y] \in \sum_{\sigma \in \mathbb{Z}^{\nu}} \mathcal{G}_{\sigma}+\sum_{r=1}^{m-1} \sum_{\sigma \in \mathbb{Z}^{\nu}} \mathcal{V}_{\sigma}^{r} .
$$

Therefore $[a, x]=[a, y]=0$ for all $a \in \mathcal{G}$ and so $x=y=0$. This completes the proof.

Theorem 2.2 together with Lemma 2.10 allows us to identify

$$
\mathcal{L} / Z(\mathcal{L})=\sum_{\sigma \in \mathbb{Z}^{\nu}} \mathcal{G}_{\sigma}+\sum_{r=1}^{m-1} \sum_{\sigma \in \mathbb{Z}^{\nu}} \mathcal{V}_{\sigma}^{r}+\mathcal{D}^{\prime} \text { where } \mathcal{D}^{\prime}=(\mathcal{Z}+\mathcal{D}) / Z(\mathcal{L})
$$

It follows from Proposition 2.11 that $\mathcal{L} / Z(\mathcal{L})$ with induced Lie bracket $[\cdot, \cdot]^{-}$is a centerless $B_{\ell}$-graded Lie algebra. So $\mathcal{L} / Z(\mathcal{L})$ admits an induced weight space decomposition relative to $H$ as $\mathcal{L} / Z(\mathcal{L})=\bigoplus_{\alpha \in \Phi}(\mathcal{L} / Z(\mathcal{L}))_{\alpha}$. We shall keep the same notation for the images of $e_{i}, f_{i}, h_{i}, h_{i, j}^{ \pm}$and $v^{r}$ in $\mathcal{L} / Z(\mathcal{L})$. Using (1.16) together with (2.5), we have an epimorphism

$$
\begin{gathered}
\psi^{\prime}: \mathcal{L} / Z(\mathcal{L}) \longrightarrow \mathfrak{A} / Z(\mathfrak{A})=\left(\mathcal{G} \otimes A_{[\nu]}^{m-1}\right) \oplus\left(\mathcal{V} \otimes A_{[\nu]}^{m-1}\right) \oplus \mathcal{D}_{A_{[\nu]}^{m-1}, A_{[\nu]}^{m-1}}, \\
\text { such that for } 1 \leq i \leq \ell, 1 \leq j \leq \nu \text { and } 1 \leq r \leq m-1 \text { we have } \\
e_{i} \mapsto e_{i} \otimes 1, f_{i} \mapsto f_{i} \otimes 1, h_{i} \mapsto h_{i} \otimes 1, h_{i, j}^{ \pm} \mapsto h_{i} \otimes t_{j}^{ \pm 1}, v_{1} \mapsto v_{1} \otimes w_{r} .
\end{gathered}
$$

Now let $\alpha, \beta, \alpha+\beta \in \Phi^{\times}, \gamma, \alpha+\gamma \in \Phi_{s h}, \sigma, \tau \in \mathbb{Z}^{\nu}$ and $1 \leq r \leq m-1$. It follows using Propositions 2.2, 2.4, (2.3) and (1.10) that $\psi^{\prime}\left(\left(\mathcal{G}_{\sigma}\right)_{\alpha}\right)=\mathcal{G}_{\alpha} \otimes t^{\sigma}$ and $\psi^{\prime}\left(\left(\mathcal{V}_{\sigma}^{r}\right)_{\gamma}\right)=\mathcal{V}_{\gamma} \otimes t^{\sigma} w_{r}$. Therefore by (1.10), we have

$$
\begin{aligned}
& \psi^{\prime}\left(\left[\left(\mathcal{G}_{\sigma}\right)_{\alpha},\left(\mathcal{G}_{\tau}\right)_{\beta}\right]^{-}\right)=\left[\psi^{\prime}\left(\left(\mathcal{G}_{\sigma}\right)_{\alpha}\right), \psi^{\prime}\left(\left(\mathcal{G}_{\tau}\right)_{\beta}\right)\right]=\mathcal{G}_{\alpha+\beta} \otimes t^{\sigma+\tau} \text { and } \\
& \psi^{\prime}\left(\left[\left(\mathcal{G}_{\sigma}\right)_{\alpha},\left(\mathcal{V}_{\tau}^{r}\right)_{\gamma}\right]^{-}\right)=\left[\psi^{\prime}\left(\left(\mathcal{G}_{\sigma}\right)_{\alpha}\right), \psi^{\prime}\left(\left(\mathcal{V}_{\tau}^{r}\right)_{\gamma}\right)\right]=\mathcal{V}_{\alpha+\gamma} \otimes t^{\sigma+\tau} w_{r} .
\end{aligned}
$$


This implies that

$$
\begin{gathered}
{\left[\left(\mathcal{G}_{\sigma}\right)_{\alpha},\left(\mathcal{G}_{\tau}\right)_{\beta}\right]^{-} \neq 0 \quad \text { and } \quad\left[\left(\mathcal{G}_{\sigma}\right)_{\alpha},\left(\mathcal{V}_{\tau}^{r}\right)_{\gamma}\right]^{-} \neq 0} \\
\text { if } \alpha, \beta, \alpha+\beta \in \Phi^{\times}, \gamma, \alpha+\gamma \in \Phi_{s h}, \sigma, \tau \in \mathbb{Z}^{\nu}, 1 \leq r \leq m-1 .
\end{gathered}
$$

Next we want to define a $\mathbb{Z}^{\nu}$-grading on $\mathcal{L}$. We recall that $\left\{\tau_{r}=\left(n_{1}^{r}, \ldots, n_{\nu}^{r}\right) \mid\right.$ $1 \leq r \leq m-1\}$ is a set of some representatives of nonzero cosets of $2 \mathbb{Z}^{\nu}$ in $\mathbb{Z}^{\nu}$. We now define a $\mathbb{Z}^{\nu}$-grading on the free Lie algebra generated by $(2.1)$ as follows:

$$
\begin{gathered}
\operatorname{deg}\left(e_{i}\right)=\operatorname{deg}\left(f_{i}\right)=\operatorname{deg}\left(h_{i}\right)=0, \operatorname{deg} v^{r}=\tau_{r}, \operatorname{deg}\left(h_{i, j}^{ \pm}\right)=2 \sigma_{j}^{ \pm}, \\
1 \leq i \leq \ell, 1 \leq j \leq \nu, 1 \leq r \leq m-1
\end{gathered}
$$

Since relations $(\mathrm{R} 1)-(\mathrm{R} 9)$ are generated by homogenous elements, (2.50) defines a $\mathbb{Z}^{\nu}$-grading on $\mathcal{L}$ and so $\mathcal{L} / Z(\mathcal{L})$ has a natural $\mathbb{Z}^{\nu}$-grading $\mathcal{L} / Z(\mathcal{L})=$ $\bigoplus_{\sigma \in \mathbb{Z}^{\nu}}(\mathcal{L} / Z(\mathcal{L}))^{\sigma}$. Now set

$$
(\mathcal{L} / Z(\mathcal{L}))_{\alpha}^{\sigma}:=(\mathcal{L} / Z(\mathcal{L}))_{\alpha} \cap(\mathcal{L} / Z(\mathcal{L}))^{\sigma} ; \quad \alpha \in \Phi, \sigma \in \mathbb{Z}^{\nu}
$$

One can use Propositions 2.2 and 2.4 to conclude that $\mathcal{V}_{\sigma}^{r} \subseteq(\mathcal{L} / Z(\mathcal{L}))^{2 \sigma+\tau_{r}}$ and $\mathcal{G}_{\sigma} \subseteq(\mathcal{L} / Z(\mathcal{L}))^{2 \sigma}$ for $\sigma \in \mathbb{Z}^{\nu}$ and $1 \leq r \leq m-1$. Therefore (2.48) together with Lemma 2.10 implies that

$$
\begin{gathered}
(\mathcal{L} / Z(\mathcal{L}))_{\alpha}^{2 \sigma}=\left(\mathcal{G}_{\sigma}\right)_{\alpha} \text { and }(\mathcal{L} / Z(\mathcal{L}))_{\beta}^{2 \sigma+\tau_{r}}=\left(\mathcal{V}_{\sigma}^{r}\right)_{\beta} \\
\alpha \in \Phi^{\times}, \beta \in \Phi_{s h}, \sigma \in \mathbb{Z}^{\nu}, 1 \leq r \leq m-1,
\end{gathered}
$$

and so

$$
(\mathcal{L} / Z(\mathcal{L}))_{\alpha}=\sum_{\sigma \in \mathbb{Z}^{\nu}}(\mathcal{L} / Z(\mathcal{L}))_{\alpha}^{\sigma} ; \quad \alpha \in \Phi^{\times}
$$

Since $\mathcal{L} / Z(\mathcal{L})$ is a centerless $B_{\ell}$-graded Lie algebra, Theorem 1.1 guarantees the existence of a unital commutative associative algebra $A$, an $A$-module $B$ and a symmetric $A$-bilinear form $g^{\prime}: B \times B \longrightarrow A$ such that

$$
\mathcal{L} / Z(\mathcal{L})=T\left(\operatorname{Cliff}(u) / \mathbb{C}, \operatorname{Cliff}\left(g^{\prime}\right) / A\right)=(\mathcal{G} \otimes A) \oplus(\mathcal{V} \otimes B) \oplus \mathcal{D}_{B, B}
$$

in which $\mathcal{G}=\mathcal{G} \otimes 1$ and the Lie bracket on $\mathcal{L} / Z(\mathcal{L})$ is given by $(1.10)$. It is easy to check that

$$
(\mathcal{L} / Z(\mathcal{L}))_{\alpha}= \begin{cases}\mathcal{G}_{\alpha} \otimes A & \alpha \in \Phi_{l g} \\ \left(\mathcal{G}_{\alpha} \otimes A\right)+\left(\mathcal{V}_{\alpha} \otimes B\right) & \alpha \in \Phi_{s h}\end{cases}
$$

Now let $\alpha \in \Phi_{l g}$ and $\sigma \in \mathbb{Z}^{\nu}$. We know that $\operatorname{dim}\left(\mathcal{G}_{\sigma}\right)_{\alpha}=\operatorname{dim}\left(\mathcal{G}_{\alpha}\right)=1$. So by (2.51) and (2.54), we can find a one-dimensional subspace $A_{\alpha}^{2 \sigma}$ of $A$ such that 
$(\mathcal{L} / Z(\mathcal{L}))_{\alpha}^{2 \sigma}=\left(\mathcal{G}_{\sigma}\right)_{\alpha}=\mathcal{G}_{\alpha} \otimes A_{\alpha}^{2 \sigma}$. Next let $\alpha, \beta \in \Phi_{l g}$ be such that $\alpha-\beta \in \Phi^{\times}$, then since $\varphi_{\sigma}: \mathcal{G} \longrightarrow \mathcal{G}_{\sigma}$ is a $\mathcal{G}$-module isomorphism, (1.10) and Lemma 2.10 imply that

$$
\begin{aligned}
\mathcal{G}_{\alpha} \otimes A_{\alpha}^{2 \sigma}=\varphi_{\sigma}\left(\left[\mathcal{G}_{\alpha-\beta}, \mathcal{G}_{\beta}\right]\right)=\left[\mathcal{G}_{\alpha-\beta}, \varphi_{\sigma}\left(\mathcal{G}_{\beta}\right)\right] & =\left[\mathcal{G}_{\alpha-\beta},\left(\mathcal{G}_{\sigma}\right)_{\beta}\right] \\
& =\left[\mathcal{G}_{\alpha-\beta},\left(\mathcal{G}_{\sigma}\right)_{\beta}\right]^{-} \\
& =\left[\mathcal{G}_{\alpha-\beta}, \mathcal{G}_{\beta} \otimes A_{\beta}^{2 \sigma}\right]^{-} \\
& =\mathcal{G}_{\alpha} \otimes A_{\beta}^{2 \sigma}
\end{aligned}
$$

so $A_{\alpha}^{2 \sigma}=A_{\beta}^{2 \sigma}$. Using [AG, (5.11)], we get $A_{\alpha}^{2 \sigma}=A_{\beta}^{2 \sigma}$ for all $\alpha, \beta \in \Phi_{l g}$ and $\sigma \in \mathbb{Z}^{\nu}$. Set

$$
A^{2 \sigma}:=A_{\alpha}^{2 \sigma} \text { for } \sigma \in \mathbb{Z}^{\nu} \text { and any choice of } \alpha \in \Phi_{l g} .
$$

Then we have

$$
(\mathcal{L} / Z(\mathcal{L}))_{\alpha}^{2 \sigma}=\left(\mathcal{G}_{\sigma}\right)_{\alpha}=\mathcal{G}_{\alpha} \otimes A^{2 \sigma} ; \sigma \in \mathbb{Z}^{\nu}, \alpha \in \Phi_{l g} .
$$

Now let $\alpha \in \Phi_{s h}$ and consider $\beta \in \Phi_{l g}$ such that $\alpha-\beta \in \Phi^{\times}$, then for all $\sigma \in \mathbb{Z}^{\nu}$, by (2.51), Lemma 2.10, (2.55) and (1.10), we have

$$
\begin{aligned}
(\mathcal{L} / Z(\mathcal{L}))_{\alpha}^{2 \sigma}=\varphi_{\sigma}\left(\left[\mathcal{G}_{\alpha-\beta}, \mathcal{G}_{\beta}\right]\right)=\left[\mathcal{G}_{\alpha-\beta}, \varphi_{\sigma}\left(\mathcal{G}_{\beta}\right)\right] & =\left[\mathcal{G}_{\alpha-\beta},\left(\mathcal{G}_{\sigma}\right)_{\beta}\right] \\
& =\left[\mathcal{G}_{\alpha-\beta},\left(\mathcal{G}_{\sigma}\right)_{\beta}\right]^{-} \\
& =\left[\mathcal{G}_{\alpha-\beta}, \mathcal{G}_{\beta} \otimes A^{2 \sigma}\right]^{-} \\
& =\mathcal{G}_{\alpha} \otimes A^{2 \sigma}
\end{aligned}
$$

This together with (2.51) and (2.55) implies that

$$
(\mathcal{L} / Z(\mathcal{L}))_{\alpha}^{2 \sigma}=\left(\mathcal{G}_{\sigma}\right)_{\alpha}=\mathcal{G}_{\alpha} \otimes A^{2 \sigma} ; \sigma \in \mathbb{Z}^{\nu}, \alpha \in \Phi^{\times} .
$$

Next let $1 \leq r \leq m-1, \alpha \in \Phi_{s h}$ and $\sigma \in \mathbb{Z}^{\nu}$. Since $\operatorname{dim} \mathcal{V}_{\alpha}=\operatorname{dim}\left(\mathcal{V}_{\sigma}^{r}\right)_{\alpha}=$ $1,(2.51)$ and (2.54) imply that there exists a one-dimensional subspace $B_{\alpha}^{2 \sigma+\tau_{r}}$ of $B$ such that $(\mathcal{L} / Z(\mathcal{L}))_{\alpha}^{2 \sigma+\tau_{r}}=\left(\mathcal{V}_{\sigma}^{r}\right)_{\alpha}=\mathcal{V}_{\alpha} \otimes B_{\alpha}^{2 \sigma+\tau_{r}}$. If $\sigma \in \mathbb{Z}^{\nu}$ and $\alpha, \beta \in \Phi_{\text {sh }}$ such that $\alpha-\beta \in \Phi^{\times}$, then considering (2.32), (2.34), Lemma 2.10 and (1.10), we have

$$
\begin{aligned}
\mathcal{V}_{\alpha} \otimes B_{\alpha}^{2 \sigma+\tau_{r}}=\psi_{\sigma}^{r}\left(\mathcal{G}_{\alpha-\beta} \cdot \mathcal{V}_{\beta}\right)=\left[\mathcal{G}_{\alpha-\beta}, \psi_{\sigma}^{r}\left(\mathcal{V}_{\beta}\right)\right] & =\left[\mathcal{G}_{\alpha-\beta}, \psi_{\sigma}^{r}\left(\mathcal{V}_{\beta}\right)\right]^{-} \\
& =\left[\mathcal{G}_{\alpha-\beta}, \mathcal{V}_{\beta} \otimes B_{\beta}^{2 \sigma+\tau_{r}}\right]^{-} \\
& =\mathcal{G}_{\alpha-\beta} \cdot \mathcal{V}_{\beta} \otimes B_{\beta}^{2 \sigma+\tau_{r}} \\
& =\mathcal{V}_{\alpha} \otimes B_{\beta}^{2 \sigma+\tau_{r}}
\end{aligned}
$$


which implies that $B_{\alpha}^{2 \sigma+\tau_{r}}=B_{\beta}^{2 \sigma+\tau_{r}}$. Use [AG, (5.11)] to conclude $B_{\alpha}^{2 \sigma+\tau_{r}}=$ $B_{\beta}^{2 \sigma+\tau_{r}}$ for all $\alpha, \beta \in \Phi_{\text {sh }}$ and define

$B^{2 \sigma+\tau_{r}}:=B_{\alpha}^{2 \sigma+\tau_{r}}$ for $1 \leq r \leq m-1, \sigma \in \mathbb{Z}^{\nu}$ and any choice of $\alpha \in \Phi_{s h}$.

Therefore by (2.51), we have

$$
(\mathcal{L} / Z(\mathcal{L}))_{\alpha}^{2 \sigma+\tau_{r}}=\left(\mathcal{V}_{\sigma}^{r}\right)_{\alpha}=\mathcal{V}_{\alpha} \otimes B^{2 \sigma+\tau_{r}} ; \sigma \in \mathbb{Z}^{\nu}, 1 \leq r \leq m-1, \alpha \in \Phi_{s h} .
$$

Since $\mathcal{G} \otimes A=\bigoplus_{\sigma \in \mathbb{Z}^{\nu}} \mathcal{G}_{\sigma}$ and $\mathcal{V} \otimes B=\bigoplus_{r=1}^{m-1} \bigoplus_{\sigma \in \mathbb{Z}^{\nu}} \mathcal{V}_{\sigma}^{r}$, (2.54) together with (2.52), (2.56) and (2.57) implies that

$$
A=\bigoplus_{\sigma \in \mathbb{Z}^{\nu}} A^{2 \sigma} \text { and } B=\bigoplus_{\sigma \in \mathbb{Z}^{\nu}} \bigoplus_{r=1}^{m-1} B^{2 \sigma+\tau_{r}}
$$
with one-dimensional summands.

Now let $\sigma, \tau \in \mathbb{Z}^{\nu}$ and $1 \leq r \leq m-1$, then for $\alpha, \beta \in \Phi^{\times}$such that $\alpha+\beta \in \Phi^{\times}$, (2.49), (2.56) and (1.10) imply that

$$
\begin{aligned}
& 0 \neq\left[\left(\frac{\mathcal{L}}{Z(\mathcal{L})}\right)_{\alpha}^{2 \sigma},\left(\frac{\mathcal{L}}{Z(\mathcal{L})}\right)_{\beta}^{2 \tau}\right]^{-} \subset\left(\frac{\mathcal{L}}{Z(\mathcal{L})}\right)_{\alpha+\beta}^{2 \sigma+2 \tau}=\mathcal{G}_{\alpha+\beta} \otimes A^{2 \sigma+2 \tau}, \\
& {\left[\left(\frac{\mathcal{L}}{Z(\mathcal{L})}\right)_{\alpha}^{2 \sigma},\left(\frac{\mathcal{L}}{Z(\mathcal{L})}\right)_{\beta}^{2 \tau}\right]^{-}=\left[\mathcal{G}_{\alpha} \otimes A^{2 \sigma}, \mathcal{G}_{\beta} \otimes A^{2 \tau}\right]^{-}=\mathcal{G}_{\alpha+\beta} \otimes A^{2 \sigma} \cdot A^{2 \tau}}
\end{aligned}
$$

and for $\alpha \in \Phi_{l g}$ and $\beta \in \Phi_{s h}$ such that $\alpha+\beta \in \Phi_{s h},(2.49),(2.57)$ and (1.10) imply that

$$
\begin{aligned}
& 0 \neq\left[\left(\frac{\mathcal{L}}{Z(\mathcal{L})}\right)_{\alpha}^{2 \sigma},\left(\frac{\mathcal{L}}{Z(\mathcal{L})}\right)_{\beta}^{2 \tau+\tau_{r}}\right]^{-} \subset\left(\frac{\mathcal{L}}{Z(\mathcal{L})}\right)_{\alpha+\beta}^{2 \sigma+2 \tau+\tau_{r}}=\mathcal{V}_{\alpha+\beta} \otimes B^{2 \sigma+2 \tau+\tau_{r}} \\
& {\left[\left(\frac{\mathcal{L}}{Z(\mathcal{L})}\right)_{\alpha}^{2 \sigma},\left(\frac{\mathcal{L}}{Z(\mathcal{L})}\right)_{\beta}^{2 \tau+\tau_{r}}\right]^{-}=\left[\mathcal{G}_{\alpha} \otimes A^{2 \sigma}, \mathcal{V}_{\beta} \otimes B^{2 \tau+\tau_{r}}\right]^{-}=\mathcal{V}_{\alpha+\beta} \otimes A^{2 \sigma} \cdot B^{2 \tau+\tau_{r}}}
\end{aligned}
$$

Therefore the one-dimensionality of the summands in (2.58) gives that

$$
\begin{aligned}
& \text { (i) } A^{2 \sigma} \cdot A^{2 \tau}=A^{2 \sigma+2 \tau} ; \sigma, \tau \in \mathbb{Z}^{\nu}, \\
& \text { (ii) } A^{2 \sigma} \cdot B^{2 \tau+\tau_{r}}=B^{2(\sigma+\tau)+\tau_{r}} ; \sigma, \tau \in \mathbb{Z}^{\nu}, 1 \leq r \leq m-1 .
\end{aligned}
$$

Using [BGKN, Lemma 1.8] together with (2.58) and (2.59)(i), we conclude that $A$ is graded isomorphic to $A_{[\nu]}$. So $A$ is a unital commutative associative algebra generated by a generating set $\left\{x_{j}^{ \pm 1} \mid 1 \leq j \leq \nu\right\}$ satisfying $x_{j} x_{j}^{-1}=1$. Let $1 \leq j \leq \nu$ and consider (2.14), then by (2.56) and Proposition 2.1, we have $\varphi_{j}^{+}\left(e_{\theta}\right) \in \varphi_{j}^{+}\left(\mathcal{G}_{\theta}\right)=\left(\mathcal{G}_{\sigma_{j}^{+}}\right)_{\theta}=\mathcal{G}_{\theta} \otimes A^{2 \sigma_{j}^{+}}$and $\varphi_{j}^{-}\left(f_{\theta}\right) \in \mathcal{G}_{-\theta} \otimes A^{2 \sigma_{j}^{-}}$. Therefore there exist $n_{j}, m_{j} \in \mathbb{C}$ such that $\varphi_{j}^{+}\left(e_{\theta}\right)=n_{j} e_{\theta} \otimes x_{j}$ and $\varphi_{j}^{-}\left(f_{\theta}\right)=m_{j} f_{\theta} \otimes x_{j}^{-1}$. 
Using (1.10) together with (2.10), the Jacobi identity, (2.16), (2.15)(iv), (2.2) and $(\mathrm{R} 3)(i)$, we have

$$
\begin{aligned}
n_{j} m_{j} h_{\theta} & =\left[n_{j} e_{\theta} \otimes x_{j}, m_{j} f_{\theta} \otimes x_{j}^{-1}\right]^{-}=\left[\varphi_{j}^{+}\left(e_{\theta}\right), \varphi_{j}^{-}\left(f_{\theta}\right)\right]^{-} \\
& =\frac{1}{2}\left[\varphi_{j}^{+}\left(h_{\theta}\right), e_{\theta}, \varphi_{j}^{-}\left(f_{\theta}\right)\right]^{-}-\frac{1}{2}\left[e_{\theta}, \varphi_{j}^{+}\left(h_{\theta}\right), \varphi_{j}^{-}\left(f_{\theta}\right)\right]^{-} \\
& =\frac{1}{2}\left[\varphi_{j}^{+}\left(h_{\theta}\right), \varphi_{j}^{-}\left(h_{\theta}\right)\right]^{-}+\frac{1}{4}\left[e_{\theta}, \varphi_{j}^{+}\left(h_{\theta}\right), \varphi_{j}^{-}\left(h_{\theta}\right), f_{\theta}\right]^{-} \\
& =0+\left[e_{\theta}, f_{\theta}\right]^{-}=h_{\theta} .
\end{aligned}
$$

Therefore $n_{j} m_{j}=1$ and so we may identify $A$ with $A_{[\nu]}$ via $t_{j}^{ \pm 1}=n_{j}^{ \pm 1} x_{j}^{ \pm 1}$. Thus it follows using $(2.15)(i v)$ and (1.10) that $k_{j}^{ \pm}=h_{\theta} \otimes t_{j}^{ \pm 1}$. Now let $1 \leq i \leq \ell$ and $1 \leq j \leq \nu$. Using $(2.56)$, we have $\varphi_{j}^{ \pm}\left(e_{i}\right) \in\left(\mathcal{G}_{\sigma_{j}^{ \pm}}\right)_{\alpha_{i}}=\mathcal{G}_{\alpha_{i}} \otimes \mathbb{C} t_{j}^{ \pm 1}$, so there exists $s_{i} \in \mathbb{C}$ such that $\varphi_{j}^{ \pm}\left(e_{i}\right)=s_{i} e_{i} \otimes t_{j}^{ \pm 1}$. Therefore by (2.15)(iii) and (2.10), we have

$$
h_{i, j}^{ \pm}=\varphi_{j}^{ \pm}\left(h_{i}\right)=\left[\varphi_{j}^{ \pm}\left(e_{i}\right), f_{i}\right]=s_{i} h_{i} \otimes t_{j}^{ \pm 1} .
$$

Thus by (2.2), we have

$$
\begin{aligned}
\left(h_{1}+\sum_{t=1}^{\ell-1} 2 h_{t}+h_{\ell}\right) \otimes t_{j}^{ \pm 1}=h_{\theta} \otimes t_{j}^{ \pm 1} & =k_{j}^{ \pm}=h_{1, j}^{ \pm}+\sum_{t=1}^{\ell-1} h_{t, j}^{ \pm}+h_{\ell, j}^{ \pm} \\
& =\left(s_{1} h_{1}+\sum_{t=1}^{\ell-1} 2 s_{t} h_{t}+s_{\ell} h_{\ell}\right) \otimes t_{j}^{ \pm 1}
\end{aligned}
$$

which implies that $s_{t}=1$ for $1 \leq t \leq \ell$. Therefore we have

$$
h_{i, j}^{ \pm}=h_{i} \otimes t_{j}^{ \pm 1} ; 1 \leq i \leq \ell, 1 \leq j \leq \nu .
$$

This together with (R7), (1.10) and Lemma 2.10 implies that

$$
\left[v^{r}, v_{2}^{s}\right]^{-}=\delta_{r, s} e_{\varepsilon_{1}+\varepsilon_{2}} \otimes t^{\tau_{r}} ; 1 \leq r, s \leq m-1 .
$$

Now let $1 \leq r \leq m-1$. Use (2.57) and fix a choice of $0 \neq \beta_{r} \in B^{\tau_{r}}$ such that $v^{r}=v_{1} \otimes \beta_{r} \in\left(\mathcal{V}_{0}^{r}\right)_{\varepsilon_{1}}$. Then (2.58) and (2.59) imply that $B$ is a free $A$-module with basis $\left\{\beta_{1}, \ldots, \beta_{m-1}\right\}$. Therefore we may assume $B=A_{[\nu]}^{m-1}$ and identify $\beta_{i}$ with $w_{i}$ for $1 \leq i \leq m-1$ where $\left\{w_{i} \mid 1 \leq i \leq m-1\right\}$ is the standard basis for $A_{[\nu]}^{m-1}$ over $A_{[\nu]}$. Thus (2.3) together with Lemma 2.10 and (1.10) implies that

$$
v_{j}^{r}=v_{j} \otimes w_{r} ; 1 \leq r \leq m-1,1 \leq j \leq 2 \ell+1 .
$$

Also by (2.53), we have

$$
\mathcal{L} / Z(\mathcal{L})=T\left(\operatorname{Cliff}(u) / \mathbb{C}, \operatorname{Cliff}\left(g^{\prime}\right) / A_{[\nu]}\right) .
$$


Therefore (2.61) together with (1.10) and (1.9) gives that

$$
\begin{aligned}
\delta_{r, s} e_{\varepsilon_{1}+\varepsilon_{2}} \otimes t^{\tau_{r}}=\left[v^{r}, v_{2}^{s}\right]^{-}=\left[v_{1} \otimes w_{r}, v_{2} \otimes w_{s}\right]^{-} & =d_{v_{1}, v_{2}} \otimes g^{\prime}\left(w_{r}, w_{s}\right) \\
& =e_{\varepsilon_{1}+\varepsilon_{2}} \otimes g^{\prime}\left(w_{r}, w_{s}\right),
\end{aligned}
$$

for $1 \leq r, s \leq m-1$, so $g^{\prime}\left(w_{r}, w_{s}\right)=\delta_{r, s} t^{\tau_{r}}$, i.e. $g^{\prime}=g$ (see (1.15)), therefore

$$
\mathcal{L} / Z(\mathcal{L})=T\left(\operatorname{Cliff}(u) / \mathbb{C}, \operatorname{Cliff}(g) / A_{[\nu]}\right) .
$$

Now we are ready to prove our main theorem which states that $\mathcal{L}$ is the universal covering algebra of $T\left(\operatorname{Cliff}(u) / \mathbb{C}, \operatorname{Cliff}(g) / A_{[\nu]}\right)$.

Proof of Theorem 2.1. Let $\pi_{1}: \mathcal{L} \longrightarrow \mathcal{L} / Z(\mathcal{L})$ and $\pi_{2}: \mathfrak{A} \longrightarrow \mathfrak{A} / Z(\mathfrak{A})$ be the natural canonical maps. Considering (2.5), (2.60) and using Lemma 2.10 , we have $\pi_{2} \psi=\pi_{1}$. Therefore $\psi: \mathcal{L} \longrightarrow \mathfrak{A}$ is an epimorphism whose kernel is a subset of $Z(\mathcal{L})$. So $\mathcal{L}$ is a central extension of $\mathfrak{A}$. But $\mathfrak{A}$ is the universal covering algebra of $T\left(\operatorname{Cliff}(u) / \mathbb{C}, \operatorname{Cliff}(g) / A_{[\nu]}\right)$ and $\mathcal{L}$ is perfect (Proposition 2.11), therefore by [MP, Proposition 1.9.3], $\mathcal{L} \cong \mathfrak{A}$.

Remark 3. In what we did, we constructed a finite presentation of the universal covering algebra of a Lie torus of type $B_{\ell}$ whose corresponding pair is $\left(S, 2 \mathbb{Z}^{\nu}\right)$ with $S \neq 2 \mathbb{Z}^{\nu}$. The same proofs as in the text shows that the finitely presented Lie algebra generated by $\left\{e_{i}, f_{i}, h_{i}, h_{i, j}^{ \pm} \mid 1 \leq i \leq \ell, 1 \leq j \leq\right.$ $\nu\}$ subject to the relations (R1), (R3), (R4), (R6)(i), (R8) and (R9) is the universal covering algebra of a Lie torus of type $B_{\ell}$ whose corresponding pair is $\left(2 \mathbb{Z}^{\nu}, 2 \mathbb{Z}^{\nu}\right)$.

\section{Acknowledgements}

The result of this work forms a part of the author's Ph.D. thesis at the university of Isfahan. The author thanks her supervisor, Professor Saeid Azam, for his continuous guidance and encouragement. The author also would like to thank Professor Erhard Neher for some helpful discussion.

\section{References}

[AABGP] B. Allison, S. Azam, S. Berman, Y. Gao and A. Pianzola, Extended affine Lie algebras and their root systems, Mem. Amer. Math. Soc. 126 (1997), no. 603, $\mathrm{x}+122 \mathrm{pp}$.

[ABG] B. Allison, G. Benkart and Y. Gao, Central extensions of Lie algebras graded by finite root systems, Math. Ann. 316 (2000), no. 3, 499-527.

[AG] B. Allison and Y. Gao, The root system and the core of an extended affine Lie algebra, Selecta Math. (N.S.) 7 (2001), no. 2, 149-212. 
[BZ] G. Benkart and E. Zelmanov, Lie algebras graded by finite root systems and intersection matrix algebras, Invent. Math. 126 (1996), no. 1, 1-45.

[BGKN] S. Berman, Y. Gao, Y. Krylyuk and E. Neher, The alternative torus and the structure of elliptic quasi-simple Lie algebras of type $A_{2}$, Trans. Amer. Math. Soc. 347 (1995), no. 11, 4315-4363.

[Bo] N. Bourbaki, Groups et algebras de Lie, Paris: Hermann, 1968. chs. 4,5,6.

[Ch] C. Chevalley, Sur certains groupes simples, Tôhoku Math. J. (2) 7 (1955), 14-66.

[G] H. Garland, The arithmetic theory of loop algebras, J. Algebra 53 (1978), no. 2, $480-551$.

[H] J. E. Humphreys, Introduction to Lie algebras and representation theory, Springer, New York, 1972

[J] N. Jacobson, Lie algebras, Interscience Publishers (a division of John Wiley \& Sons), New York, 1962.

[K] C. Kassel, Kähler differentials and coverings of complex simple Lie algebras extended over a commutative algebra, J. Pure Appl. Algebra 34 (1984), no. 2-3, 265-275.

[MP] R. V. Moody and A. Pianzola, Lie algebras with triangular decompositions, Wiley, New York, 1995

[MRY] R. V. Moody, S. E. Rao and T. Yokonuma, Toroidal Lie algebras and vertex representations, Geom. Dedicata 35 (1990), no. 1-3, 283-307.

[SaY] K. Saito and D. Yoshii, Extended affine root system. IV. Simply-laced elliptic Lie algebras, Publ. Res. Inst. Math. Sci. 36 (2000), no. 3, 385-421.

[St] R. Steinberg, Générateurs, relations et revêtements de groupes algébriques, in Colloq. Théorie des Groupes Algébriques (Bruxelles, 1962), 113-127, Librairie Universitaire, Louvain.

[Ya] H. Yamane, A Serre-type theorem for the elliptic Lie algebras with rank $\geq 2$, Publ. Res. Inst. Math. Sci. 40 (2004), no. 2, 441-469.

[Yo1] Y. Yoshii, Root systems extended by an abelian group and their Lie algebras, J. Lie Theory 14 (2004), no. 2, 371-394.

[Yo2] - Lie tori, simple charactrization of extended affine Lie algebars, preprint. 